\title{
RECHERGHES SUR LA RÉCOLTE DU POLLEN PAR LES ABEILLES (Apis Mellifica L) (Fin)
}

PAR

\section{Jean LOUVEA UX}

Station de Recherches Apicoles

Bures-sur-Yvette (Seine-et-Oise)

\section{Recherches sur l'origine des variations qualitatives}

Les premières observations de I950 nous ont done amenés à constater qu'il existe une importante variation qualitative dans les récoltes de pollen des différentes colonies. Chaque ruche a sa façon particulière d'exploiter la flore locale mais nos observations ne permettent toutefois pas de dégager des lois générales permettant de comprendre l'origine des variations qualitatives constatées.

Nous avons tout d'abord pensé que l'hétérogénéité des courbes constatée en I950 pouvait tout simplement provenir du fait que nous avions utilisé des colonies quelconques d'origine mal définie. Il convenait donc d'opérer une nouvelle série d'essais sur des ruches plus homogènes.

\section{A. - VARIATION QUALITATIVE CHEZ DES RUCHES HOMOG ENES}

Pour vérifier notre hypothèse notre premier travail fut de constituer un lot de ruches aussi homogènes que possible à partir des colonies disponibles. Dans le courant du printemps 1952 des reines italiennes de premier choix provenant directement d'un élevage réputé de Bologne furent introduites dans 5 bonnes ruches en remplacement de reines noires ou métisses qui s'y trouvaient. Au fur et à mesure de la ponte de la reine italienne les abeilles noires se trouvaient progressivement éliminées et en 1953 nous étions en possession de 5 colonies totalement italianisées et relativement homogènes.

Les trappes à pollen ayant été installées dès les premiers jours de récolte nous avons procédé à l'analyse des échantillons de pollen recueillis dans la période du I4 mars au 30 avril I953 par la méthode du triage 
des pelotes. I,es analyses n'ont porté que sur les journées les plus importantes et à des intervalles de 2 à 3 jours. Wne analyse quoticlienne ne nous a pas paru, en effet, rigoureusement indispensable; nous avons toutefois noté chaque jour le sens général de l'évolution de la formule pollinique ; cette évolution ayant un caractère assez progressif les courbes obtenues restent valables et comparables à celles de i 950 .

Pour représenter l'évolution de la récolte nous avous adopté la même convention graphique qu'en i950. I a comparaison des graphiques concernant les récoltes de I950 et I953 nous permet de constater que des ruches homogènes par l'origine de la reine présentent encore des diffírences importantes dans la jormule pollinique adoptée (fig. 13). Nous noterons que les plantes visitées par les abeilles sont peu différentes d'une année à l'autre. Quelques pollens manquent en I953, année plus tardire que I950 ; c'est le cas de Populus tremula et de Populus nigra; Taraxacum est très peu visité en I953. Par contre Acer est visité en 1953 et lle l'arait pas été en I950.

Pour faciliter la comparaison des deux séries de graphiques et la ramener a une comparaison de chiffres nous arons eu l'idée de planimétrer les surfaces occupées par chaque pollen sur les graphiques. Nous avons ensuite ramené les chiffres obtenus exprimés en $\mathrm{cm}^{2}$ à un pourcentage de la surface totale du graphique. I.e tableau IX fournit les données ainsi obtenues et montre nettement la variabilité des récoltes dans le cas de raches homogines.

\section{B. - DURABILITÉ DES PRÉFÉRENCES MANIFESTÉES PAR CERTAINES RUCHES POUR CERTAINS POLLENS}

Ayant montré que de's ruches apparemment homogènes présentent d's préférences diverses pour certains pollens nous avons roulu rechercher si ces préférences correspondent à un caractère fixé dans le patrimoine. héréditaire de la colonie on bien s'il s'agit d'un phénomène accidentel sujet à des fluctuations inportantes d'année en année, donc totalement indépendant de tout facteur d'ordre génétique.

Pour pouvoir mettre en évidence nue continuité éventuclle des préférences des diverses colonies nous arons maintenu en io5t le dispositif expérinental établi en I953 an 1noyen des ruches 6, I05, 108, 109, I46. Ces ruches ont été laissées en place sans aucune modification et les récoltes de I954 ont été analysées dans les neèmes conditions qu'en I05.3. Nous avous établi les mêmes graphiques (fig. 44) et opéré le même planinétrage des surfaces occupées par les différents pollens (tableau IX).

I a comparaison des récoltes peut se faire aisément sur les graphiques 

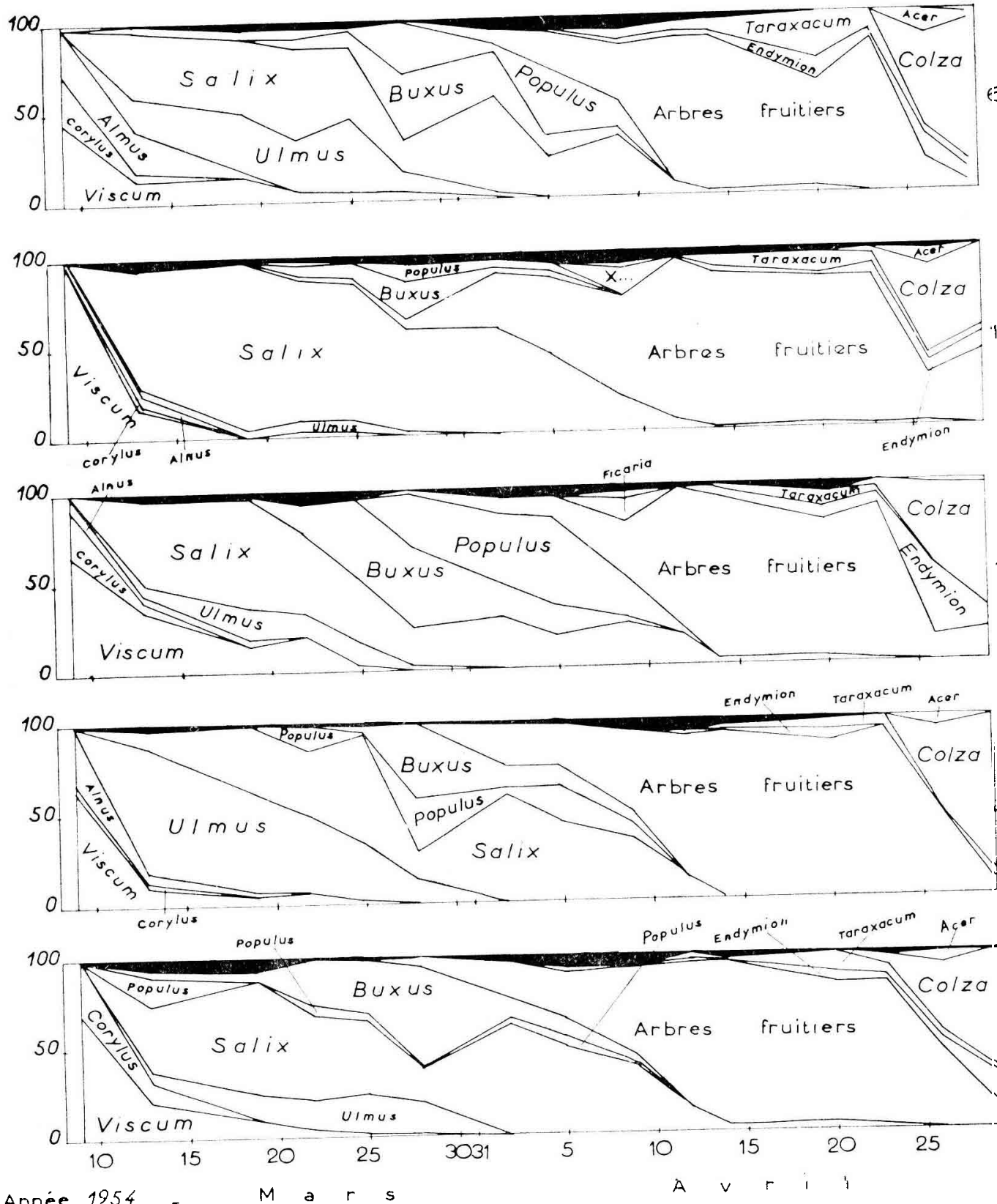

FIG. 44. 
(I, I959) RECHERCHES SUR LA RÉCOLTE DU POLLEN

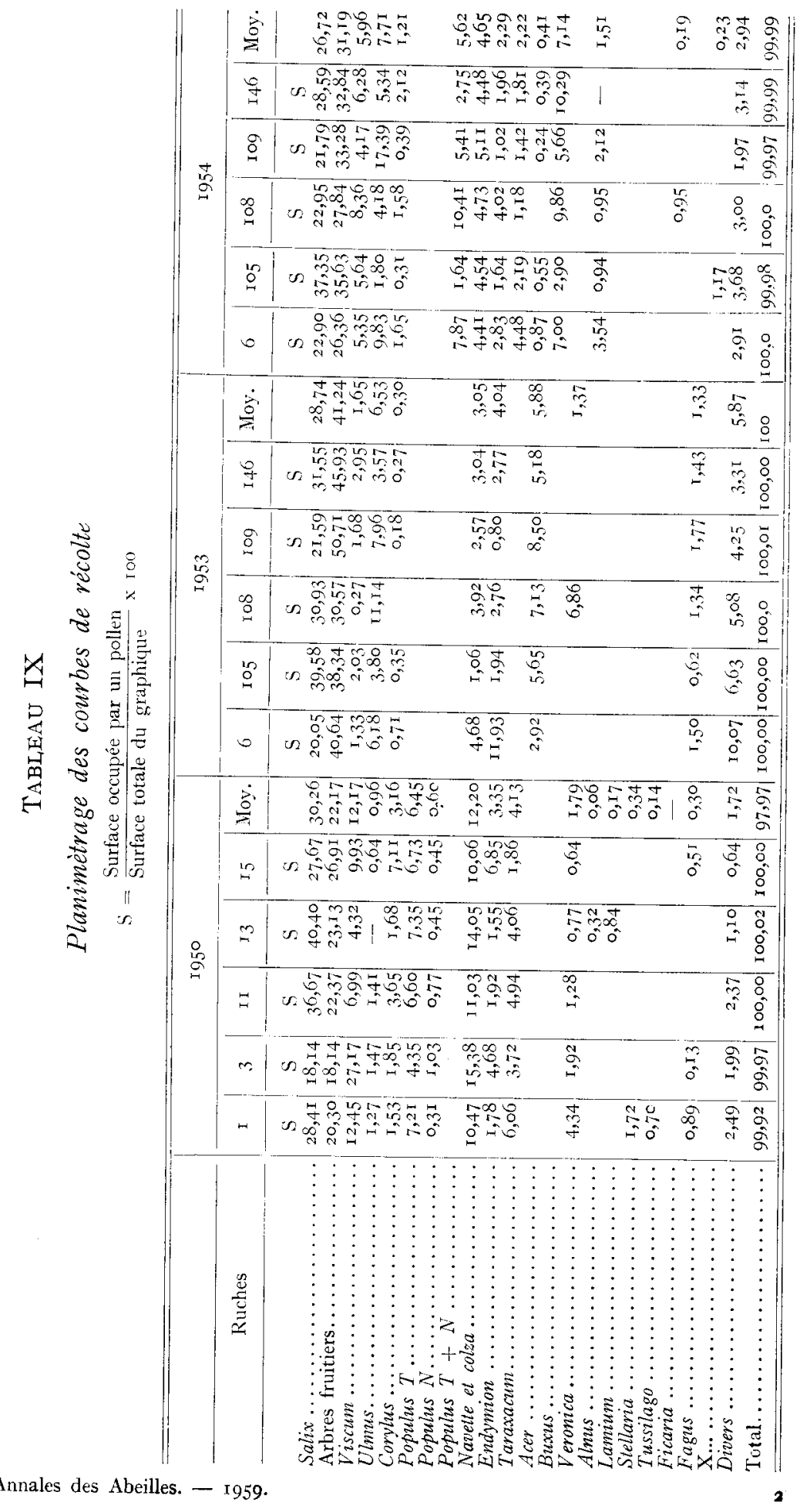


45,46 et 47 qui groupent les résultats du planimétrage pour les plantes principales. (L'année I950 est ajoutée à titre comparatif sur la fig. 45.) Dans ces graphiques la hauteur des colonnes est proportionnelle à la surface $S$ déterminée par planimétrage. On a :

$$
S(\text { pollen } X)=\frac{\text { surface occupcée par le pollen } X}{\text { Surface totale du graphique }} \times \text { roo. }
$$

Le trait pointillé horizontal indique la valeur de la moyenne. I,e tableau IX donne les valeurs numériques exactes des pourcentages que représentent les colonnes.

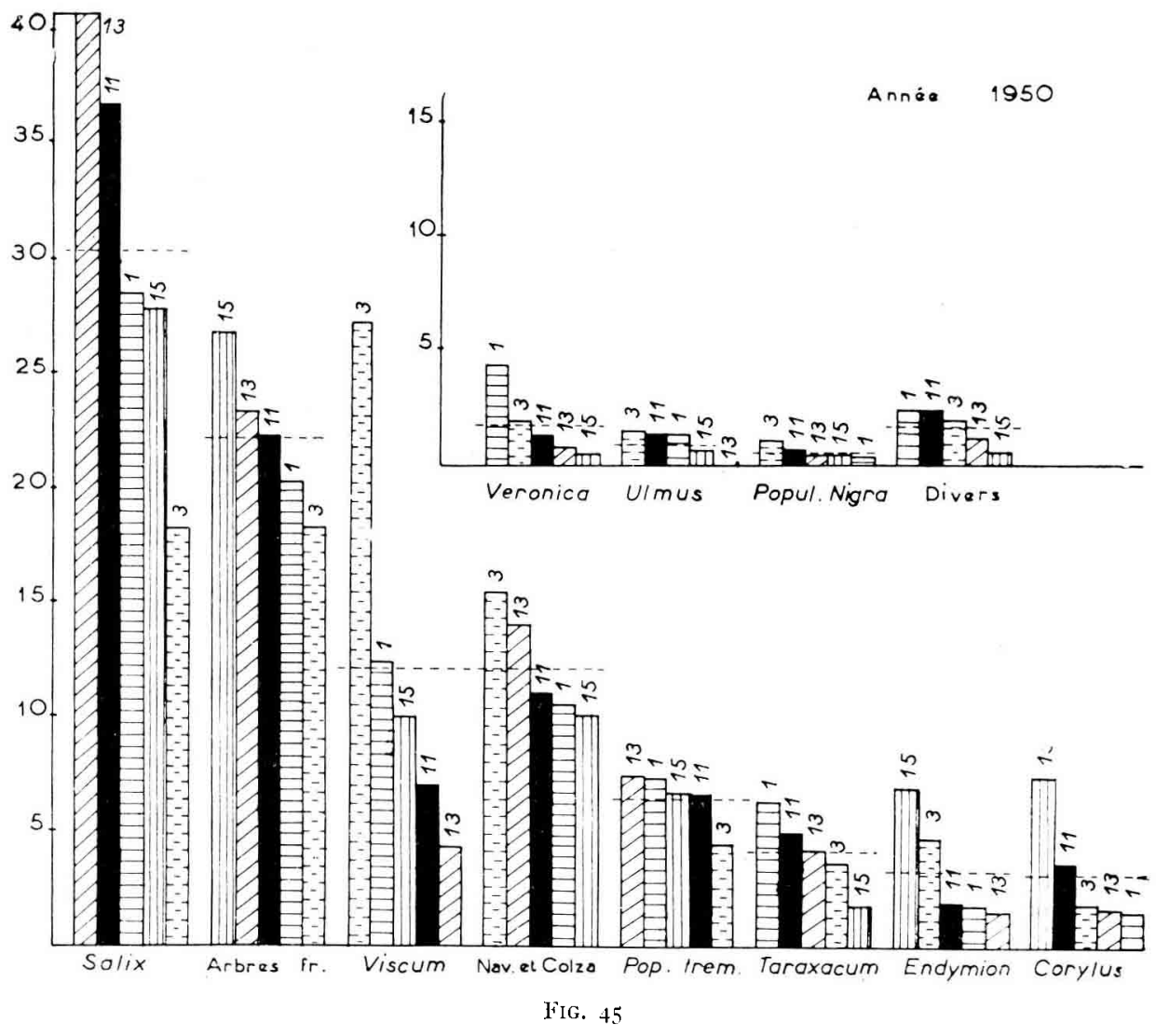

Nous constatons que pour Salix l'ordre d'importance des pourcentages des différentes ruches est presque le même pour les deux années; seules les ruches 6 et rog voient leur ordre inversé mais les différences de valeur des pourcentages sont très faibles. On peut donc considérer que d'une année à l'autre on retrouve dans le groupe de ruches des valeurs similaires pour le pourcentage du pollen de Saule, ce qui indiquerait une certaine continuité dans les goûts manifestés par les différentes colonies 


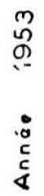

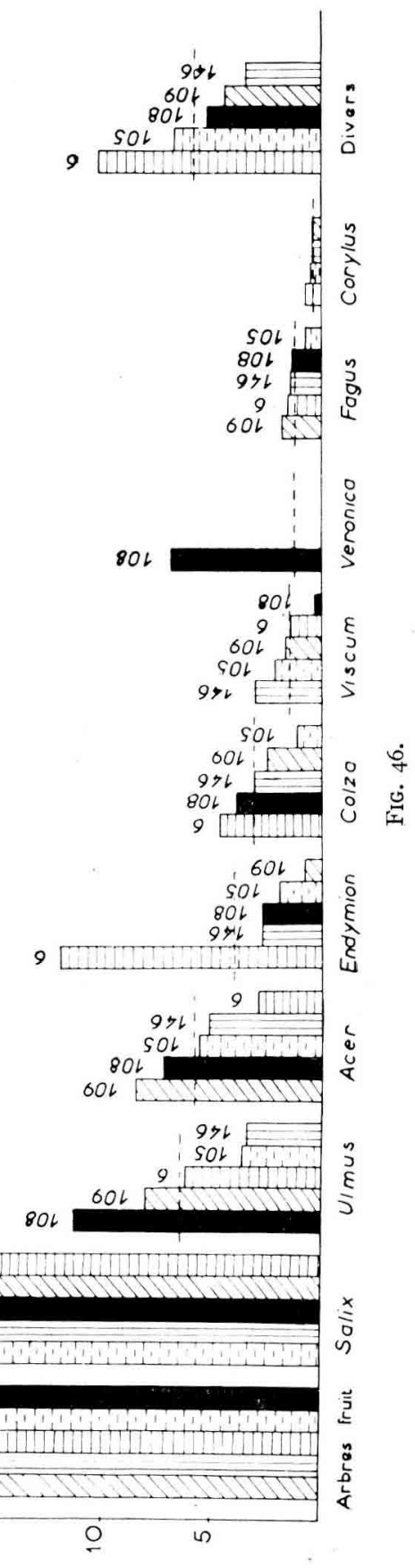


pour des pollens déterminés. Bien que le phénomène soit moins net, on le retrouve pour les arbres fruitiers : les ruches rog et I 46 se retrouvent dans les deux années avec de forts pourcentages, les ruches 6 et ro8 avec de faibles pourcentages. I a ruche I05 seule change de position et passe de faibles pourcentages en I955 à un fort pourcentage en I954.

Pour les autres plantes les conclusions sont difficiles à tirer; il ne semble pas y avoir continuité dans le choix. Pour essayer d'améliorer les

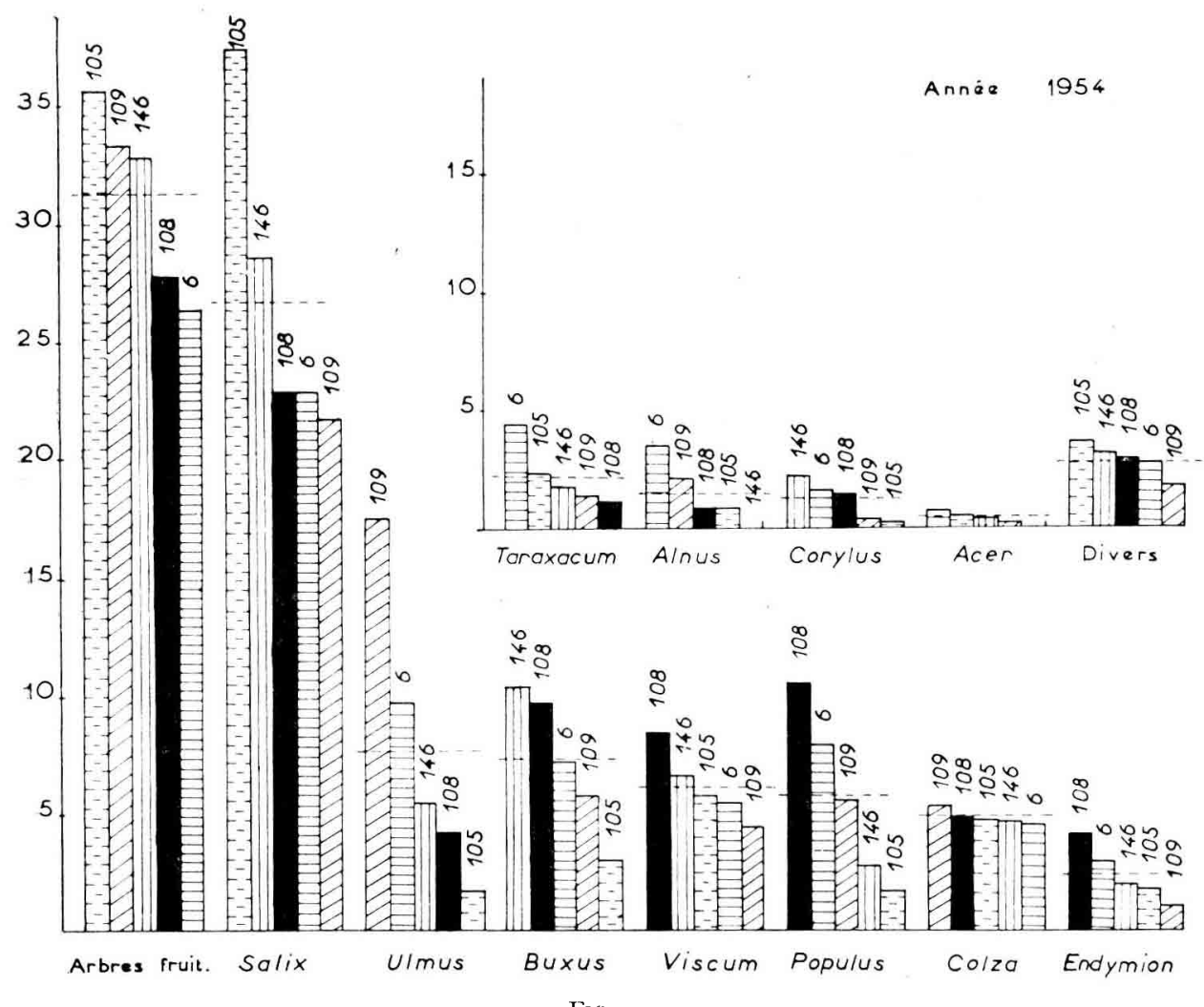

FIG. 47 .

bases de comparaison nous avons repris nos chiffres en tenant compte cette fois de la quantité de pollen effectivement récoltée.

Nous avons donc repris nos résultats d'analyses de I953 et I954 pour la période allant du début des récoltes jusqu'au zo avril (fig. 43 et 44) et, tenant compte de la quantité de pollen récoltée chaque jour par nos différentes ruches expérimentales, nous avons calculé le poids de chaque pollen dans la récolte totale de chaque ruche pendant la période de floraison de la plante foumissant le pollen. On a donc :

$$
\mathrm{P}(\text { pollen } \mathrm{X})=(p \times a \%)+\left(p^{\prime} \times b \%\right)+\left(p^{\prime \prime} \times c \%\right)+\cdots
$$


p, p', p" étant les poids des récoltes journalières d'une ruche, a, b, c, les pourcentages du pollen $\mathrm{X}$ dans ces récoltes. Le nombre des facteurs $p, p^{\prime}, p^{\prime \prime}$... est fonction du nombre de jours de floraison de la plante $\mathbf{X}$.

Nous obtenons ainsi le tableau $X$ qui comporte d'une part les valeurs $S$ (en p. roo) définies plus haut et les valeurs $P$ (en g) que nous venons de déterminer.

Pour chaque amnée (I953-I954) nous établissons deux colonnes, l'une où sont portées les valeurs de S S et de $\mathbf{P}$, l'autre où nous inscrivons le numéro des ruches dans l'ordre oì se présentent les différentes valeurs de $S$ et de $P$. Nous remarquerons que les valeurs de $S$ penvent présenter des divergences plus ou moins importantes par rapport aux valeurs de $\mathrm{P}$; le classement des ruches se trouve parfois modifié ; le phus souvent il s'agit d'une simple interversion.

Le calcul de $P$ confirme entièrement les conclusions tirées de l'examen des caleurs de $S$, tout au moins en ce qui concerne Salix. Pour les arbres fruitiers les valeurs de $\mathrm{P}$ s'ordonnent de la même façon dans les deux ammées à une interversion près (Io9, I46) qui d'ailleurs n'est pas très importante. Pour Ulmus 1'ordre des valeurs de $\mathrm{P}$ est aussi le même dans les deux années, toujours à une interversion près. Pour les autres plantes nous retombons dans les mêmes conclusions que précédemment avec l'examen des valuurs de $s$. Il ne semble pas y avoir continuité.

Nous arons tenté d'analyser statistiquement le's données au tableau $\mathrm{X}$. Ln calcul de $\%^{2}$ a confirmé la très nette continuité des gouts pour Salix d'une année sur l'autre. Pour les autres plantes il ne semble pas que la méthode employée permette d'améliorer beaucoup nos conclusions

Nous dirons done que d'une année sur l'autre les goûts manifjestés par une ruche pour certains pollens peuvent se retronver; toutefois, le phénomène ne semble intéresser que des plantes de toute première importance; la continuité des goûts lorsqu'il s'agit de sources de pollen d'importance secondaire est beaucoup plus discutable et n'existe sans doute même pas du tout. Ces conclusions n'impliquent pas, à notre sens, que l'abeille soit capable d'identifier le Saule botaniquement parlant. Il nous parait beaucoup plus vraisemblable qu'il existe simplement dans les différentes colonies un faiscean de chimiotropismes plus ou moins accentués qui font que le pollen de Saule présente pour certaines colonies une attractivité plus grande que pour d'autres. Nous reviendrons sur ce sujet plus 1oin. 


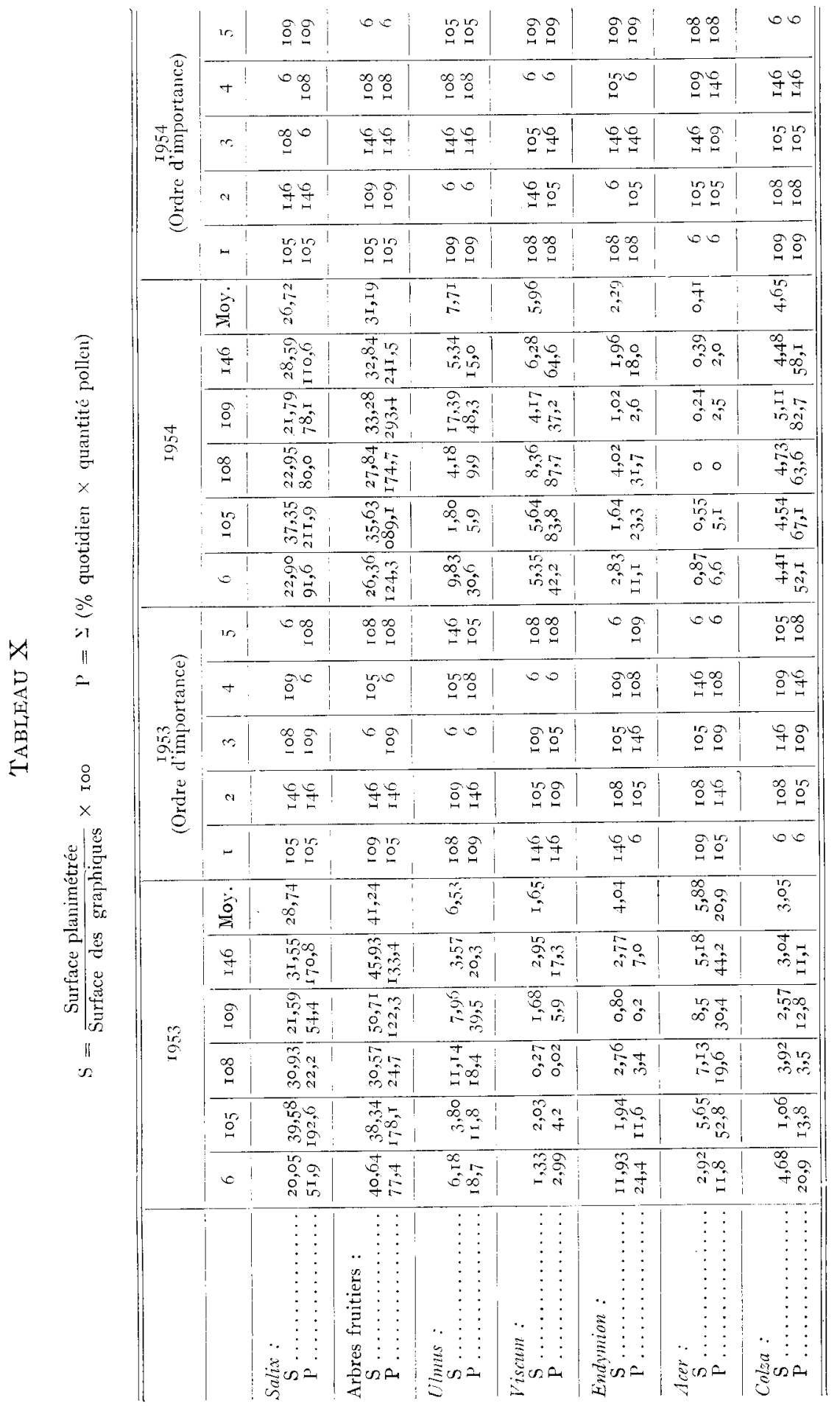




\section{C. - VARIATION QUALITATIVE EN FONCTION DE L'ORIGINE GÉOGRAPHIQUE DES COLONIES}

Il a été question plus haut, au chapitre III se rapportant à 1'étude quantitative des récoltes de pollen, d'une expérience réalisée en I955 et au cours de laquelle nous avons mis en comparaison différents groupes de ruches d'origines géographiques diverses. Nous renvoyons au chapitre II (matériel expérimental) pour la description descolonies employées, leur origine et tous leurs caractères particuliers. Nous avons mis à profit cette expérience pour faire l'étude des récoltes sur le plan qualitatif.

Il était en effet intéressant de savoir si la variabilité dans la nature botanique des récoltes de pollen ne présente pas des degrés. Nous avons donc recherché s'il n'existe pas une variabilité de faible amplitude responsable du fait que des ruches homogènes par la race et l'origine géographique présentent malgré tout des récoltes différentes, et une variabilité de plus forte amplitude ne pouvant être mise en évidence que par la comparaison de groupes de ruches homogènes, chaque groupe présentant des caractères déterminés quant à la race et l'origine géographique.

Autrement dit, nous avons émis l'hypothèse que les récoltes d'un groupe de ruches homogènes pourraient présenter une variabilité interne plus faible que la variabilité apparaissant lorsque l'on compare entre eux différents groupes de ruches, chaque groupe ayant une origine différente. Si cette hypothèse se révélait exacte nous serions en droit d'admettre que la nature des pollens récoltés est en rapport avec des facteurs liés aux caractères particuliers (race, origine géographique, etc.) de chaque groupe. Sur le plan quantitatif (chapitre III) nous avons déjà mis en évidence des différences liées à l'origine géographique des colonies.

Pour mener à bien cette expérience cruciale nous avons procédé au groupage des récoltes de nos différentes ruches semaine par semaine. En effet, il paraissait matériellement impossible de faire des analyses quotidiennes sur une vingtaine de ruches pendant plusieurs mois; un tel travail de dépouillement nous aurait occupé pendant plus d'un an en ne nous apportant d'ailleurs que des détails peu importants. Nous n'avons donc fait qu'une récolte par semaine sur chaque colonie et les analyses ont été faites, au début par la méthode du triage des pelotes et ensuite (à partir du 25 avril) par la méthode d'identification sur lame qui nous a permis un dépouillement rapide.

Comme pour les années précédentes nous avons utilisé la méthode habituelle de représentation graphique des résultats. La figure 48 nous 
donne l'allure générale des récoltes dans les différents groupes. Un examen, même sommaire, de cette figure nous permet de constater que les groupes de ruches présentent des différences réelles sur le plan de l'utilisation de la flore locale. Nous retrouvons aisément des particularités de comportement qui caractérisent le groupe car elles se retrouvent dans toutes les ruches qui le composent d'une façon assez semblable.

Ainsi, les groupes $\mathrm{J}$ et $\mathrm{P}$ semblent se concentrer sur un nombre relativement restreint de plantes essentielles alors que le groupe $\mathrm{R}$ présente une beaucoup plus grande dispersion. Certaines plantes très importantes telles que Salix, Arbres fruitiers, Trèfle incarnat, etc. sont plus visitées par les groupes $J$ et $P$ que par le groupe $R$ lequel semble s'intéresser davantage à des plantes secondaires telles que Rumex, Ranunculus, Graminées, Plantago. Il existe donc des types différents de comportement à l'égard de la flore locale dans des groupes de ruches d'origines diverses. Ce fait ressort assez nettement de la lecture des graphiques.

I1 apparaissait toutefois indispensable d'approfondir le phénomène. Quel que soit son intérêt, le mode de représentation graphique adopté manque encore beaucoup de précision puisqu'il laisse à l'œeil le soin de dégager des courbes les caractères des différents groupes. Par ailleurs, i] a l'inconvénient de donner la même importance aux périodes de faible et de forte récolte. Or, il faut bien admettre qu'il n'est pas indifférent qu'un pollen soit récolté en faible pourcentage mais en forte quantité ou en fort pourcentage et en faible quantité. Nous avons donc cherché un autre moyen de représenter 1'allure des récoltes de nos ruches pour tenir compte de tous ces éléments.

Nous avons donc établi pour chaque plante dont le pollen est, à un moment quelconque, présent dans les récoltes un tableau du type suivant :

\section{TABBLFAU XI}

\begin{tabular}{|c|c|c|c|c|c|c|}
\hline \multirow[b]{2}{*}{ Ruches } & \multicolumn{3}{|c|}{ Semaine I } & \multicolumn{3}{|c|}{ Semaine 2} \\
\hline & $\begin{array}{l}\text { Poids } \\
\text { total } \\
\text { récolté }\end{array}$ & 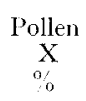 & $\begin{array}{l}\text { Poids du pollen } \\
\mathrm{X} \text { dans la récolte }\end{array}$ & $\begin{array}{l}\text { Poids } \\
\text { total } \\
\text { récolté }\end{array}$ & 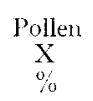 & $\begin{array}{l}\text { Poids du pollen } \\
\mathrm{X} \text { dans la récolte }\end{array}$ \\
\hline $\mathrm{R}_{\mathrm{I}} \ldots \ldots$ & A & $a$ & $\frac{\mathrm{A} \cdot \mathbf{a}}{\mathrm{r} 00}=m$ & $A^{\prime}$ & $a^{\prime}$ & $\frac{\mathrm{A}^{\prime} \cdot a^{\prime}}{\mathrm{I} 00}=m^{\prime}$ \\
\hline $\mathrm{R} 2 \ldots$ & $B$ & $b$ & $\frac{\mathrm{B} \cdot b}{\mathrm{I} 00}=n$ & $B^{\prime}$ & $b^{\prime}$ & $\frac{\mathbf{B}^{\prime} \cdot b^{\prime}}{100}=n^{\prime}$ \\
\hline
\end{tabular}

Ce tableau se prolonge évidemment vers le bas et intéresse toutes les ruches; il se prolonge vers la droite aussi longtemps qu'il est nécessaire 
pour couvrir la période de floraison de la plante fournissant le pollen $\mathrm{X}$. Un tableau est établi de même pour chaque pollen.

Si on effectue:

$$
\begin{aligned}
& \frac{m+m^{\prime}+m^{\prime \prime}+\cdots}{\Lambda+\mathrm{A}^{\prime}+\mathrm{A}^{\prime \prime}+\cdots} \times \text { I00 }=\mathrm{MP}_{\mathrm{P}} \mathrm{R}_{\mathbf{1}}(\mathrm{X}) \\
& \frac{n+n^{\prime}+n^{\prime \prime}+\cdots}{B-B^{\prime}+B^{\prime \prime}+\cdots} \times 100=\mathrm{H}_{\mathrm{P}} \mathrm{R}_{2}(\mathrm{X}) \\
& \frac{o+o^{\prime}+o^{\prime \prime}+\cdots}{\left(\mathrm{C}+\mathrm{C}^{\prime}+\mathrm{C}^{\prime \prime}+\cdots\right.} \times 100=\mathrm{U}_{\mathrm{J}}, \mathrm{K}_{3}(\mathrm{X})
\end{aligned}
$$

Mp R I (X), Mp R 2 (X), etc. sont les moyennes pondérées des pourcentages du pollen $X$ dans les récoltes des ruches $R I, R 2$, etc.

Nous pouvons ensuite déterminer la moyenne pondérée du pollen $\mathrm{X}$ dans l'ensemble des récoltes du groupe $\mathrm{R}$ ell appliquant la formule :

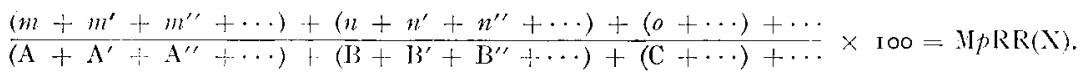

Nous obtenons finalement pour chaque pollen étudié, une série de moyennes pondérées dont les unes (Mp R I (X), Mp R $2(\mathrm{X}), \operatorname{Mp} \mathrm{R}_{3}(\mathrm{X})$ ) concernent les ruches prises individuellement et dont les autres (Mp RR $(\mathrm{X}), \mathrm{Mp} J \mathrm{~J}(\mathrm{X}), \mathrm{Mp} \mathrm{PP}(\mathrm{X})$, etc.) sont valables pour l'ensemble des ruches d'un groupe.

Nous ne donnerons pas ici le détail des 45 tableaux qui ont dî être établis sur le modèle du tableau XI. Nous avons regroupé dans le tableau XI l'ensemble des valeurs finales obtenues.

I,es groupes $\mathrm{J}, \mathrm{R}$ et $\mathrm{P}$ nous ont fourni des données s'étendant sur toute l'année apicole. I.es groupes I, et B n'ont pas été suivis aussi longtemps pour des raisons matérielles indépendantes de notre volonté. Nous avons donc établi, d'une part une moyenne arithmétique des groupes J, R et $P$ et d'autre part une moyenne de tous les groupes lorsque la chose était possible. Dans le tableau XII les plantes sont classées par ordre d'importance ou, plus exactement, dans l'ordre décroissant des valeurs cle

$$
\frac{M_{\mu R R}+M_{\mu} J+M_{1} P \mathrm{P}}{3} .
$$

Pour rendre plus lisible le vaste tableau XII qui est assez peu nuaniable nous arons repris les données qu'il contient sous forme de grapliques par plante, beaucoup plus directement lisibles. Nous en donnons quelques exemples caractéristiques parmi les 45 qui ont été établis (fig. 49). I,es valeurs portées en abscisses (o à 5o) sont les moyennes pondérées tirées du tableau XII; chaque segment horizontal correspond à 111 groupe ; le trait vertical est la valeur de la moyenne pondérée du groupe (Mp RR, Mp JJ etc.) ; les numéros portés sur les segments horizontaux sont ceux des ruches. On peut distinguer plusieurs cas : 

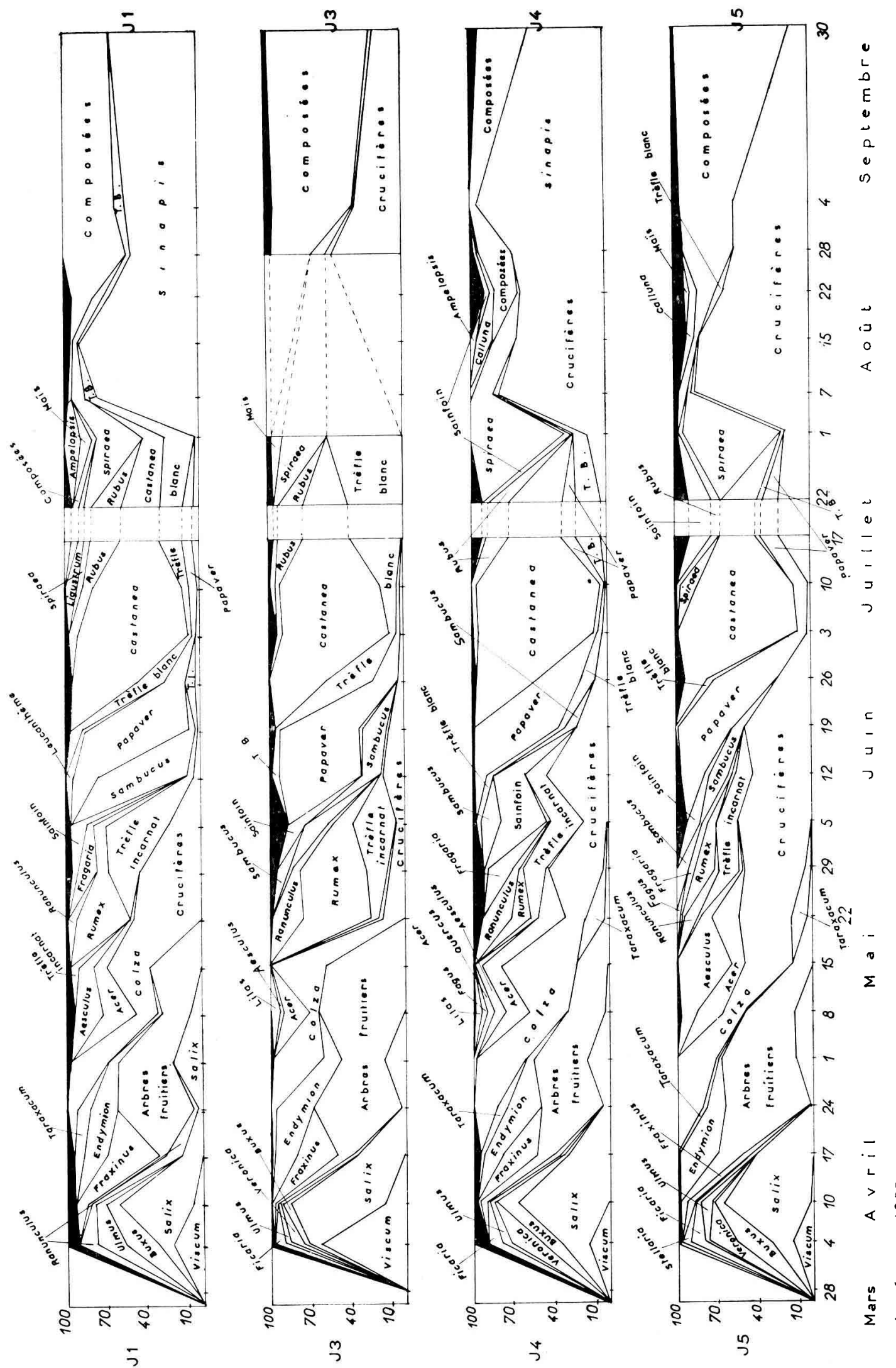


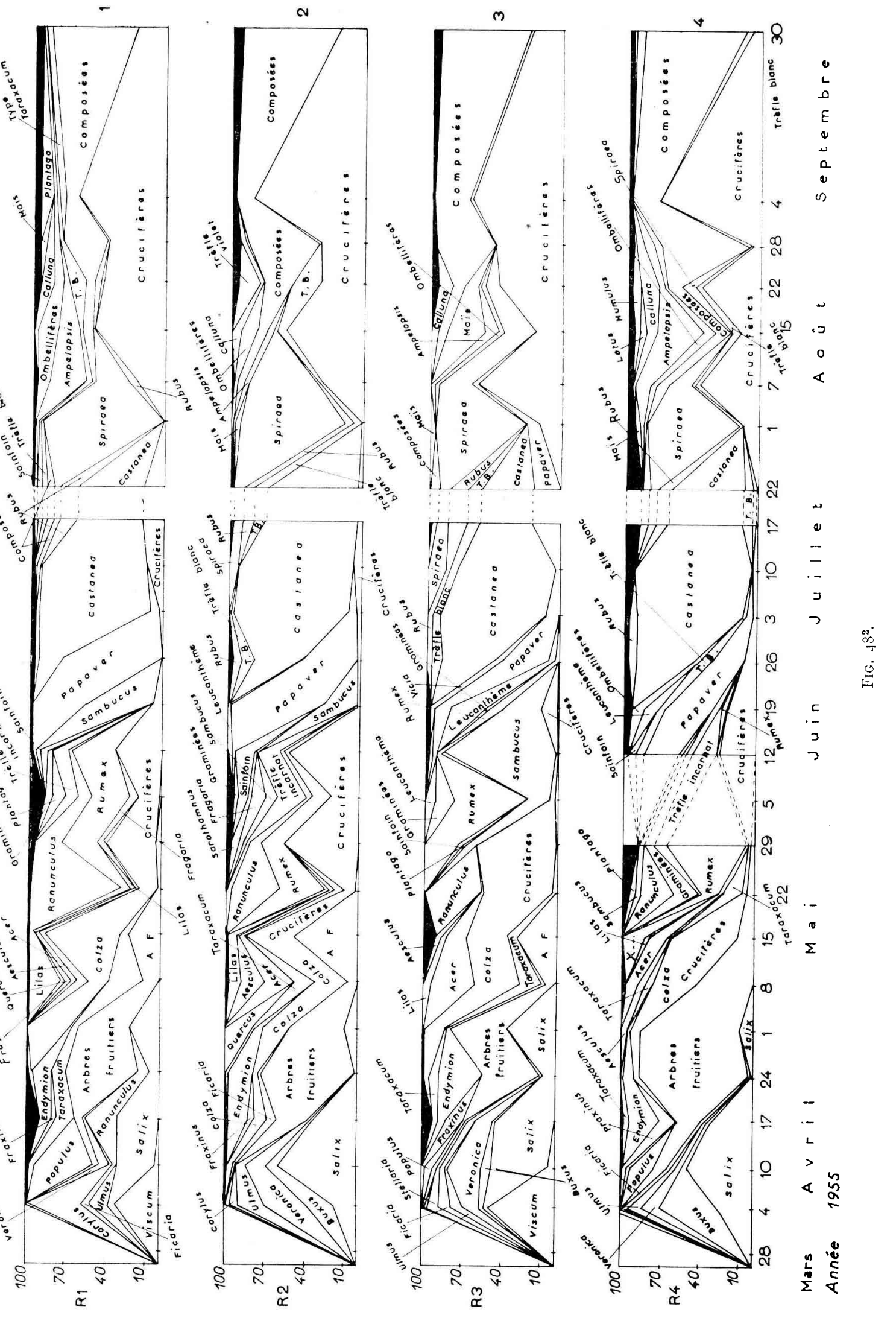




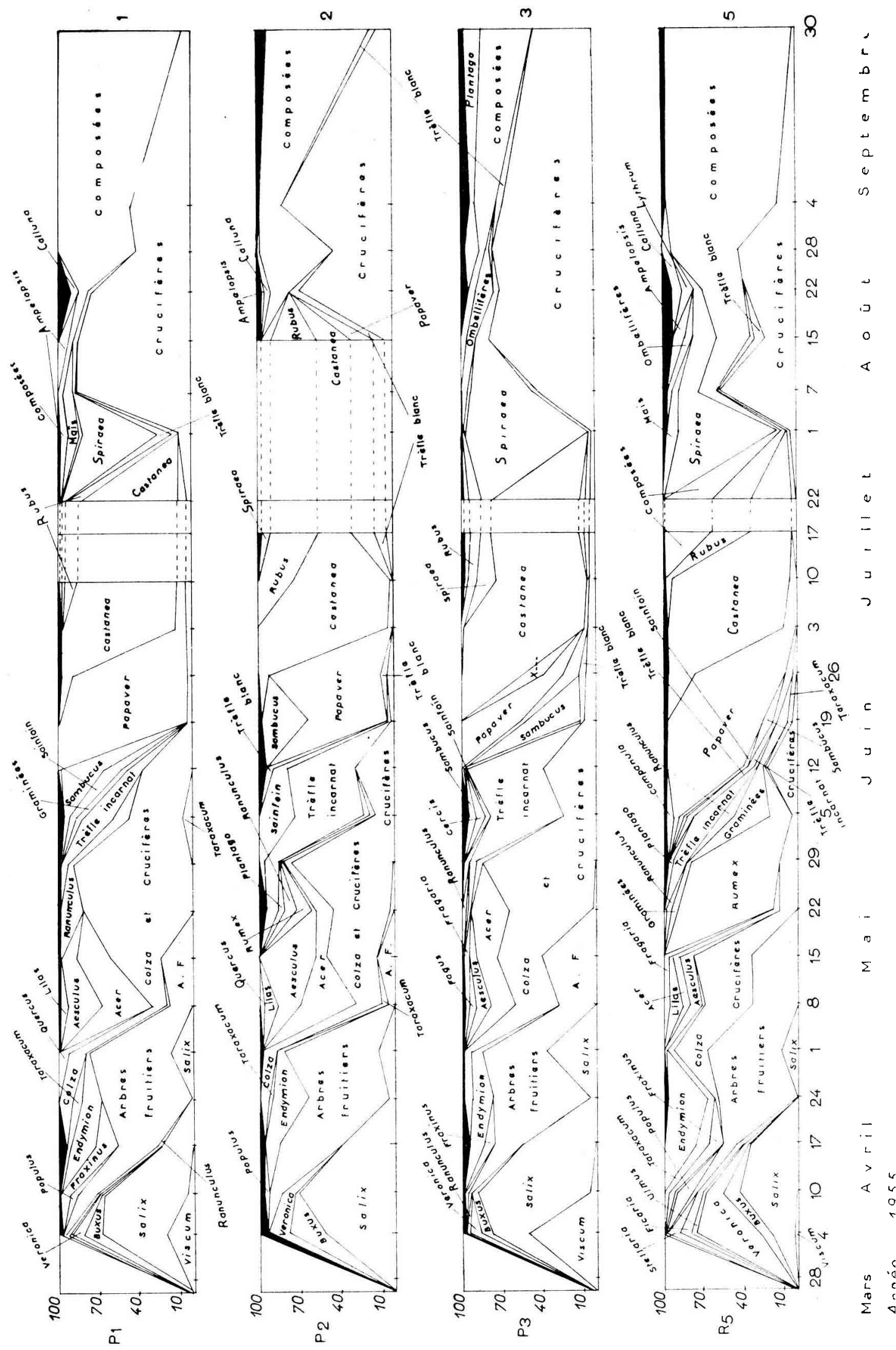



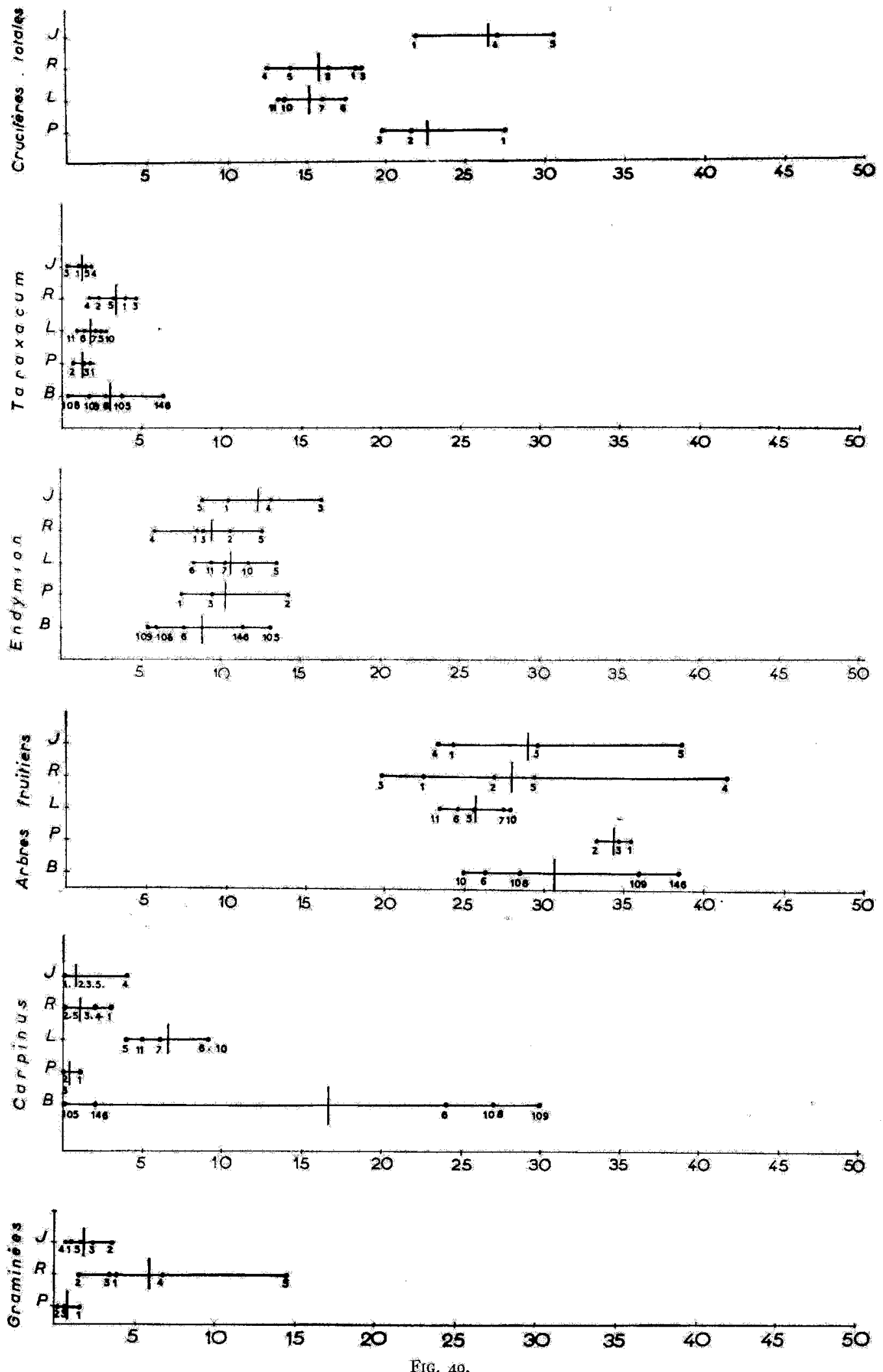
Tupe Crucifères. - I'analyse statistique des résultats (analyse de la variance par le test I; de Student-Snedecor) prouve que le hasard ne piut pas expliquer seul cette répartition. Il y a donc bien des groupes de ruches présentant des affinités plus ou moins grandes pour les Crucifères. Nous retrouvons pour les plantes suivantes des résultats du même ordre (valeur de Ii excluant le simple hasard pour une telle répartition) : Rumex, I'eronica, Taraxacum, Fragaria.

Type Endymion. - Dans ce cas il semble bien que le comportement de's différents groupes soit le même vis-à-vis de la plante. I a valeur de I trouvée, qui est très faible, confirme cette impression. I, a plante peut ètre considérée comme indifférente, les divers groupes de ruches s'y intéressant d'une façon à peu près analogue. Ia variation à l'intérieur d'un groupe est du même ordre que la variation inter-groupes. Les plantes suivantes sont à ranger dans le même groupe qu'Endymion: Papazer, Arbres fruitiers, Sambucus, Viscum, Rubus, Quercus, Lythrum, Stellaria, ('arpinus, Vicia, liagus, Betula.

Il convient de remarquer que l'analyse fait intervenir tous les groupes à la fois, c'est-à-dire qu'elle consiste à prendre pour erreur résiduelle une estimation commune des variances des divers groupes. Or ces variances sont, dans bien des cas hétérogènes. Ainsi dans le cas des Arbres fruitiers les groupes $\mathrm{I}_{\text {, et }} \mathrm{P}$ ont des variances très faibles par rapport aux autres. In test I' s'appliquant à ces 2 seuls groupes montrerait une différence hautement significative. Cependant ces variations de la variance peuvent ctre aléatoires et il est vraisemblablement plus correct de tester les différences observées par rapport à l'erreur commune.

I)ans un troisième groupe de plantes qui comprend toutes les plantes qui n’ont pas encore été citées, la valeur de I’ indique que le seuil de signification n'a pas été atteint ; mais là encore on remarquera que lorsqu'on restreint la comparaison à deux ou trois groupes on trouve des différences significatives. I, 'examen des graphiques de la figure 49 suffira à prouver que si, pour Clmus par exemple la valeur de li est inférieure au senil 1orsqu'on compare tous les groupes ensemble, on note cependant une différence évidente entre les groupes $\mathrm{L}, \mathrm{P}$ et $\mathrm{B}$.

I,e faible nombre de plantes pour lesquelles nous avons trouvé des valeurs de I' qui excluent toute intervention du hasard s'explique par le fait que sur 3 ou 5 groupes il s'en présente fréquemment un dont la variabilité interne est très grande, ou deux ou trois qui varient de la même faço11.

Les faibles valeurs de li trouvées dans un grand nombre de cas n'inpliquent pas que notre hypothèse de base soit fausse. Peut-être serionsnous arrivés à des résultats beaucoup plus nets avec un matériel biologique parfaitement homogène du point de vue génétique, mais ce n'est pas certain. Il faut dire à ce propos que la génétique de l'abeille est encore 
dans l'enfance en raison des problèmes à peu près insurmontables qu'elle pose dans l'état actuel de la science. Nous avons choisi des ruches aussi homogènes que possible parmi celles dont nous pouvions disposer; il est vraisemblable que malgré tout, ces colonies présentaient encore des différences génétiques, ce qui ne saurait nous surprendre. Il faut aussi admettre que plusieurs groupes de ruches peuvent parfaitement"se comporter de la même façon vis-à-vis de certaines plantes.

Nous dirons en conclusion de ces expériences que dans un grand nombre de cas le comportement des abeilles d'un groupe de ruches aussi homogènes que possible par la race et l'origine géographique vis-à-vis de la flore est différent du comportement des abeilles d'un autre groupe présentant une origine différente. La variabilité à l'intérieur du groupe est plus faible, très souvent, que la variabilité inter-groupes.

Les ruches d'un groupe donné peuvent présenter un comportement très homogène vis-à-vis d'une plante ou d'un ensemble de plantes mais diverget plus ou moins profondément sur d'autres points sans que nous puissions encore nous expliquer la raison de ces divergences. Peut-être certaines plantes sont-elles polyvalentes par la qualité du pollen qu'elles offrent.

\section{D. - ÉtUdE DE LA DISPERSION DU SPECTRE POLLINIQUE}

Nous avions déjà remarqué en I950 que certaines ruches tendaient toujours à présenter une formule pollinique plus compliquée que d'autres. Le tableau VIII nous avait d'ailleurs permis de dégager cette notion de façon approchée. Grâce aux chiffres plus abondants que nous possédons pour l'année I955 nous allons pouvoir nous livrer à une étude plus approfondie du phénomène en observant, non plus des ruches isolées mais des groupes de ruches de même origine.

Si nous portons sur un même graphique, en abscisses les pourcentages moyens atteints dans les récoltes par les différents pollens (moyennes générales pondérées des groupes $J, R$ et $P$, voir tableau XII) dans leur ordre croissant d'importance et, en ordonnées la valeur de ces pourcentages, nous obtenons une courbe d'allure hyperbolique (fig. 5o). L'allure générale de cette courbe traduit le fait que les pollens importants sont rares et que les pollens secondaires sont, de loin, les plus nombreux. Nous retrouvons là le même type de courbe que celles qu'on obtient en faisant l'étude des populations d'insectes capturés au moyen de pièges lumineux par exemple. On trouve dans ce cas quelques espèces très communes mais peu nombreuses et de nombreuses espèces peu communes.

D'ailleurs, si nous comparons notre graphique à la composition de la flore locale nous trouvons aussi qu'il existe effectivement quelques plantes très communes et une foule de plantes peu représentées numériquement.

Annales des Abcilles. - I959. 
TABL

\begin{tabular}{|c|c|c|c|c|c|c|c|c|c|c|c|}
\hline & \multicolumn{6}{|c|}{ Groupe J } & \multicolumn{5}{|c|}{ Groupe R } \\
\hline & $M_{\mathrm{P}}$ & $\mathrm{M}_{\mathrm{J} 2}$ & $\mathrm{M}_{3}$ & $\mathrm{Mp}_{J_{4}}$ & $\mathrm{H}_{\mathrm{P}} \mathrm{J}_{5}$ & ${ }_{\mathrm{JJ}}^{\mathrm{I}_{\mathrm{P}}}$ & ${ }^{\mathrm{Mp}_{\mathrm{P}}}$ & ${ }^{\mathrm{M}_{\mathrm{P} 2}}$ & ${ }^{\mathrm{N}_{1}} \mathrm{R}_{3}$ & ${ }^{\mathrm{N}_{\mathrm{p}}}{ }_{\mathrm{R}_{4}}$ & ${ }^{M_{P_{5}}}$ \\
\hline Castanea. & 56,98 & $\cdots$ & $54,7 \mathrm{I}$ & 64,93 & 44,74 & & 50,84 & $6=, 40$ & $6 \mathrm{I}, 52$ & & \\
\hline Arbres fruilie & $24, \mathrm{I} I$ & - & $29,4 \mathrm{I}$ & 23,14 & $\begin{array}{l}44,14 \\
38,55\end{array}$ & $\begin{array}{l}5,, 5,3 \\
29,24\end{array}$ & 22,38 & 26,88 & $\begin{array}{l}01,52 \\
19,65\end{array}$ & $\begin{array}{l}09,70 \\
41,26\end{array}$ & $\begin{array}{l}55,45 \\
20,25\end{array}$ \\
\hline Papaver Rhaeas............ & 29,08 & - & 28,69 & 29,63 & 31,72 & 29,48 & 38,66 & 25,52 & 16,04 & I 6,98 & 44,88 \\
\hline Crucifère $\ldots \ldots \ldots \ldots \ldots \ldots \ldots$ & $21,9^{6}$ & - & - & $27, \mathrm{I}$ I & 30,68 & 26,18 & I 8,18 & 16,48 & 18,36 & 12,77 & 13,98 \\
\hline Salix $\ldots \ldots \ldots \ldots \ldots \ldots \ldots$ & 18,5 & $\cdots$ & I 9,9 & I 6,5 & I 5,4 & I 7,65 & $I 5,3$ & 10,7 & 25,0 & II,O & I 2,3 \\
\hline Spiraea ulmaria .............. & 7,65 & $\mathrm{I} 3, \mathrm{OrI}$ & & 14,62 & 12,27 & I I, 22 & 6,75 & 20,22 & 22,40 & 12,77 & $I_{5}, 72$ \\
\hline Rtmex .................. & 10,0 & $\cdots$ & 34,2 & 2,10 & 8,7 & 13,90 & 24,6 & $\mathbf{1} 8,6$ & 20,6 & 27,7 & 38,6 \\
\hline$\ldots \ldots \ldots \ldots$ & 12,37 & ..-- & 7,69 & 8,04 & I8, Г4 & I 1,74 & 9,95 & I I 45 & 9,99 & 10,23 & 24,76 \\
\hline Trifolimn incarnalum......... & 18,84 & I 2,78 & 10,47 & 9,60 & 8,65 & 12,55 & 3,01 & $6,5^{6}$ & $0,3 \mathrm{I}$ & 4,55 & IO, 53 \\
\hline Acer pseudo-platanus .......... & $8,4 \mathrm{I}$ & - & 9,08 & IO,OI & 12,63 & 9,94 & 2,68 & 8,82 & I $9,1 \mathrm{I}$ & 6,19 & $3,5 \mathrm{I}$ \\
\hline Endymion nutans $\ldots \ldots \ldots \ldots \ldots$ & 10,46 & -1 & 16,20 & I3,08 & 8,76 & $\mathrm{I} I, 8 \mathrm{I}$ & 8,53 & 10,64 & 8,84 & 5,88 & 12,58 \\
\hline$\because \ldots \ldots \ldots \ldots \ldots$ & 6,40 & $8,5^{6}$ & $7,7 \mathrm{I}$ & 7, OI & 7,37 & $7,2 \mathrm{I}$ & 9,62 & 9,30 & 23,27 & 2,28 & I,94 \\
\hline$s$ excelsior.....$\ldots \ldots$ & 24,64 & & 10,66 & 9,82 & 6,56 & I 3, I 5 & $\mathrm{I}, 77$ & $\mathrm{I}, 78$ & 6,32 & 7,29 & 5,92 \\
\hline a album. & 8,2 & 6,5 & $22, I$ & 1,0 & 2,3 & I. $I, 36$ & 2,0 & 0 & 8,9 & 0 & 0,4 \\
\hline Rantunculus II & 0,62 & 2,92 & 6,90 & 4,02 & $2,5 \mathrm{I}$ & 3,35 & $20,5^{2}$ & 9,72 & $\mathrm{I}, \mathrm{I} 7$ & $3,5 \mathrm{I}$ & $5,3 \mathrm{I}$ \\
\hline Véronica ...... & 0,6 & 4,6 & 3,3 & 2,5 & $\mathrm{I}, \mathrm{I}$ & 2,08 & 4,5 & $\mathrm{I} 2, \mathrm{I}$ & 19,8 & 3,7 & I r, O \\
\hline$\ldots \ldots \ldots \ldots$ & 3,91 & & 5,87 & $3,5 \mathrm{I}$ & I,68 & 3,82 & 16,15 & 10,39 & 5,35 & $\mathrm{I}, 48$ & 5,96 \\
\hline pervivens ..... & 5,7 & $\mathbf{I} 4,6$ & 4,5 & 10,7 & 4,6 & 6,17 & 0 & 8,3 & 4,7 & 4,0 & 6,0 \\
\hline oin $\ldots \ldots \ldots \ldots \ldots \ldots \ldots$ & 5,30 & $\mathrm{I}, 28$ & 4,8 & I 2,12 & 3,43 & $6, \mathrm{TI}$ & 0,53 & $4,9 \mathrm{I}$ & 0,24 & 1,03 & 1,55 \\
\hline$\ldots \ldots \ldots \ldots \ldots$ & 7,58 & - & & 4,82 & 2,10 & $5,5 \mathrm{I}$ & 2,03 & 4,92 & $2,8 \mathrm{I}$ & 4,43 & 2,92 \\
\hline Rubus...... & 5,34 & 一 & 4,08 & 2,59 & $\mathrm{I}, 07$ & 3,50 & 2,70 & 4,75 & 3,76 & 3,12 & 5,09 \\
\hline Aesculus ... & 4,07 & 一 & 0,31 & 2,50 & 5,53 & $3, \circ 3$ & 2,25 & 3,85 & 0,37 & 3,02 & 2,99 \\
\hline Populus nigra & 0,49 & - & 0 & 0 & 0,42 & $0,2 \mathrm{r}$ & 18,42 & 0,64 & 4,45 & 10,44 & 4,82 \\
\hline Graminces. & 0,73 & 3,67 & 2,36 & 0,92 & $\mathrm{I}, 58$ & I,59: & 3,78 & $I, 49$ & 3,46 & 6,63 & 14,50 \\
\hline Ulmus & 8,92 & $5,2 \mathrm{I}$ & $\mathrm{I}, 74$ & 3,24 & 7,57 & 4,54 & $0,9 \mathbf{I}$ & 9,96 & $\mathrm{I}, 5 \mathrm{I}$ & 3,25 & $\mathrm{I}, 40$ \\
\hline Cercis: & 0,68 & 6,07 & 0,50 & $\mathrm{I}, 9 \mathrm{I}$ & $\circ$ & $\mathrm{I}, 3 \mathrm{I}$ & 3,54 & $\mathrm{I}, 25$ & 0 & 0 & 0 \\
\hline$c a \ldots \ldots \ldots$ & 6,07 & 0,54 & 6,02 & $5, \mathrm{I} 8$ & $\mathrm{I}, 72$ & 5,68 & $3,5 \circ$ & $4, \mathbf{I} 5$ & o & 0,73 & 1,52 \\
\hline$\ldots \ldots \ldots$ & 3,49 & - & - & 0,36 & $\mathrm{I}, 29$ & 1,73 & 5,88 & $\mathrm{I}, 63$ & 2,16 & 9,35 & 0,77 \\
\hline Quercus $I$ & 1,46 & - & $\mathrm{I}, 24$ & $3, \mathrm{I} 3$ & $\circ$ & $1,26 !$ & 1,24 & $\mathrm{I} 3,8 \mathrm{I}$ & 0 & $0,7^{2}$ & 0 \\
\hline garis & 0,38 & -- & - & $2,8,3$ & 2,43 & $\mathrm{I}, 8 \mathrm{I}$ & 5,34 & 2,52 & 4,34 & 7,64 & 2,47 \\
\hline Ranunculus I ............ & 6,16 & $-\cdots$ & $0,8 \mathbf{I}$ & $\mathrm{I}, 44$ & 0,74 & $2, \mathrm{II}$ & I 2, IO & 2,46 & 3,37 & $\mathrm{I}, 73$ & 2,73 \\
\hline Corylus a & 0 & $\circ$ & 0 & 0 & & $\circ$ & $4 \mathrm{I}, 0$ & 7,0 & 1,0 & 0 & $\circ$ \\
\hline cum dens-leonis .... & $\mathrm{I}, 02$ & - & 0,26 & $\mathrm{I}, 72$ & $\mathrm{I}, 65$ & $\mathrm{I}, \mathrm{I} 2$ & 3,98 & 2,39 & 4,65 & $\mathrm{r}, 78$ & $3, \mathrm{I} 3$ \\
\hline Lythrum salicaria $\ldots \ldots \ldots \ldots$ & 1,76 & - & $2, \mathrm{I} 5$ & 1,25 & $2,5^{8}$ & 1,87 & I,I9 & 1,06 & 0 & 1,72 & 4,45 \\
\hline Zea mais ......... & $I, 57$ & - & & $\circ$ & $I, 64$ & 1,04 & 0,96 & 2,08 & 6,90 & 0,96 & $4,3^{6}$ \\
\hline Ombellifères $\ldots \ldots \ldots \ldots$ & 0,26 & - & 0 & 0,14 & 0,49 & 0,22 & $2,4 \mathrm{I}$ & 0,75 & 0,67 & 3,09 & $0,2 \mathrm{I}$ \\
\hline Stellaria. & o & 0,32 & 0,44 & $\mathrm{I}, \mathrm{I} 2$ & $\mathrm{I}, 60$ & 0,65 & $0, I_{3}$ & 0 & 4,60 & $\circ$ & $\mathrm{I}, 54$ \\
\hline Leucanthemum vulgare & $0,7 \mathbf{I}$ & $0, \mathrm{I} 4$ & $\circ$ & $\circ$ & $\circ$ & 0,22 & $0,2 \mathrm{I}$ & $0,6 I$ & I 5,26 & $3,9 \mathrm{r}$ & 0,42 \\
\hline Carpinus betulus ... & 0 & $\circ$ & 0 & 4,0 & $\circ$ & 0,60 & 3,0 & 0 & 2,0 & 2,0 & 0 \\
\hline Fagus silvatica ... & $\circ$ & -- & 0 & 4,0 & o & 0,88 & $I, O$ & $\circ$ & $\circ$ & I,o & 0 \\
\hline Sarothanmus... & 0 & 1,27 & 0,98 & 0 & $\circ$ & 0,33 & 2,09 & 2,25 & o & 2,08 & 0 \\
\hline Glechoma hederacea.... & o & o & 0 & 0 & $\circ$ & 0 & o & 0 & 5,0 & 1,0 & 0 \\
\hline Vicia $\ldots \ldots \ldots \ldots \ldots$ & 0,20 & $\mathrm{o}, \mathrm{I} 3$ & 0,47 & 0,30 & $0, \mathrm{I} 7$ & 0,28 & $\mathrm{I}, 44$ & $0,5^{2}$ & $I, 06$ & 0,73 & 0,74 \\
\hline & o & $\circ$ & 0 & $\circ$ & 0 & 0 & o & 0 & & 2,0 & 0 \\
\hline Betula & 1,48 & I,49 & 0,93 & 0,54 & 0,55 & 0,98 & o & 0 & 2,88 & $\mathrm{r}, 22$ & 0,55 \\
\hline
\end{tabular}




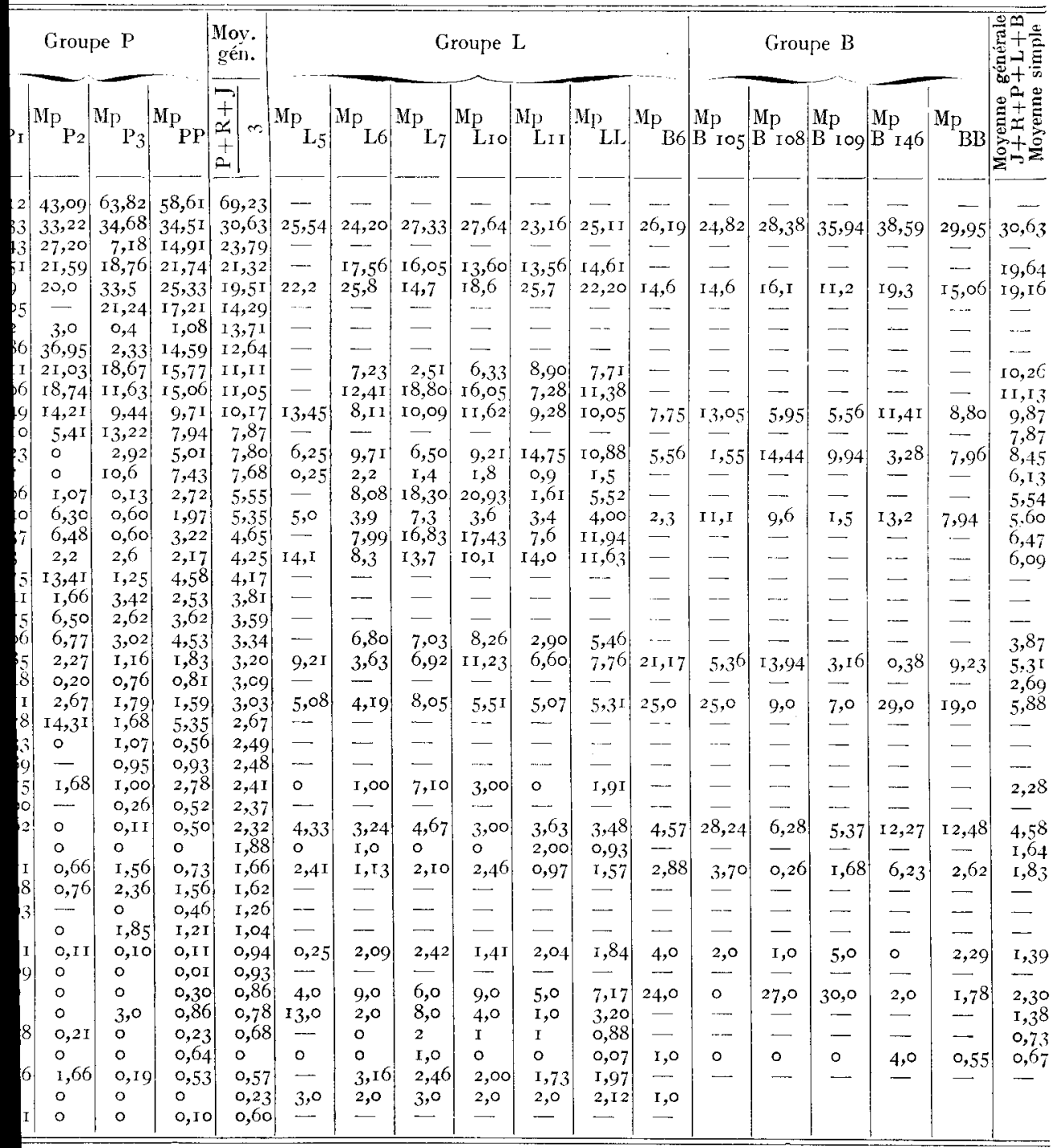


Nous pouvons, en regard de la courbe qui correspond à la moyenne

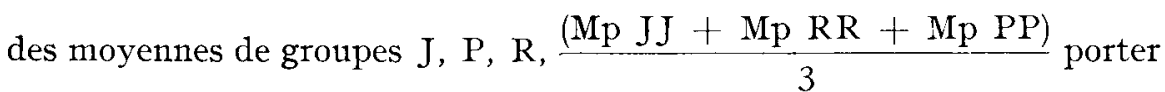
les points correspondant aux moyennes de chacun des groupes. L'ordre des plantes ne sera pas le même mais ceci importe peu puisque nous étudions la dispersion des récoltes en elle-même et non la nature botanique de cette dispersion.

Groupe J (fig. 50). - Pour ce groupe, les plantes importantes présentent des pourcentages en général supérieurs à la moyenne; les points situés sous la courbe moyenne sont rares. Au contraire la fin de la courbe passe sous la courbe moyenne et s'y maintient.

Groupe $R$ (fig. 50). -- La courbe du groupe $\mathrm{R}$ commence par être au-dessus de la moyenne, devient pratiquement tangente à cette courbe moyenne pour les pourcentages compris entre I 5 et $6 \mathrm{p}$. Ioo, puis se maintient ensuite très au-dessus de la moyenne. Pour atteindre le zéro de la courbe il faudrait la prolonger sensiblement vers la droite ce qui indique, et nous l'avons vérifié, que ce groupe visite un plus grand nombre d'espèces que les autres. Si nous avions voulu pousser l'étude du spectre pollinique jusque dans ses moindres détails nous aurions trouvé au moins Io à I5 espèces supplémentaires à ajouter pour l'achèvement de la courbe du groupe $\mathrm{R}$.

Groupe P (fig. 50). - I a courbe du groupe P se présente comne celle du groupe $J$ avec une chute encore plus accentuée vers la droite.

Ces trois courbes traduisent très fidèlement le fait que la flore n'est pas utilisée de la même façon par les trois groupes. Les groupes $J$ et $P$ limitent leurs visites à des plantes peu nombreuses qu'ils utilisent à fond tandis que les ruches du groupe $\mathrm{R}$ dispersent leurs efforts dans un nombre élevé de directions différentes.

En conclusion, nous dirons que des groupes de ruches d'origines différentes se comportent différemment vis-à-vis de la flore tant par la nature des plantes utilisées que par les proportions, dans les récoltes, des différents pollens.

\section{E. - RECHERCHE D' UNE BASE DE COMPARAISON DES GROUPES}

Le fait d'avoir établi la permanence des groupes dans le rucher montre bien l'existence de facteurs internes agissant sur le comportement des abeilles butineuses. Le milieu extérieur qui est le même pour toutes les colonies, n'est pas tout. Chaque colonie travaille et récolte le pollen en fonction d'éléments qui jusqu'ici nous restent inconnus. Pour comprendre les raisons intimes des différences de comportement constatées il nous a fallu rechercher quelle corrélation pouvait exister entre les 


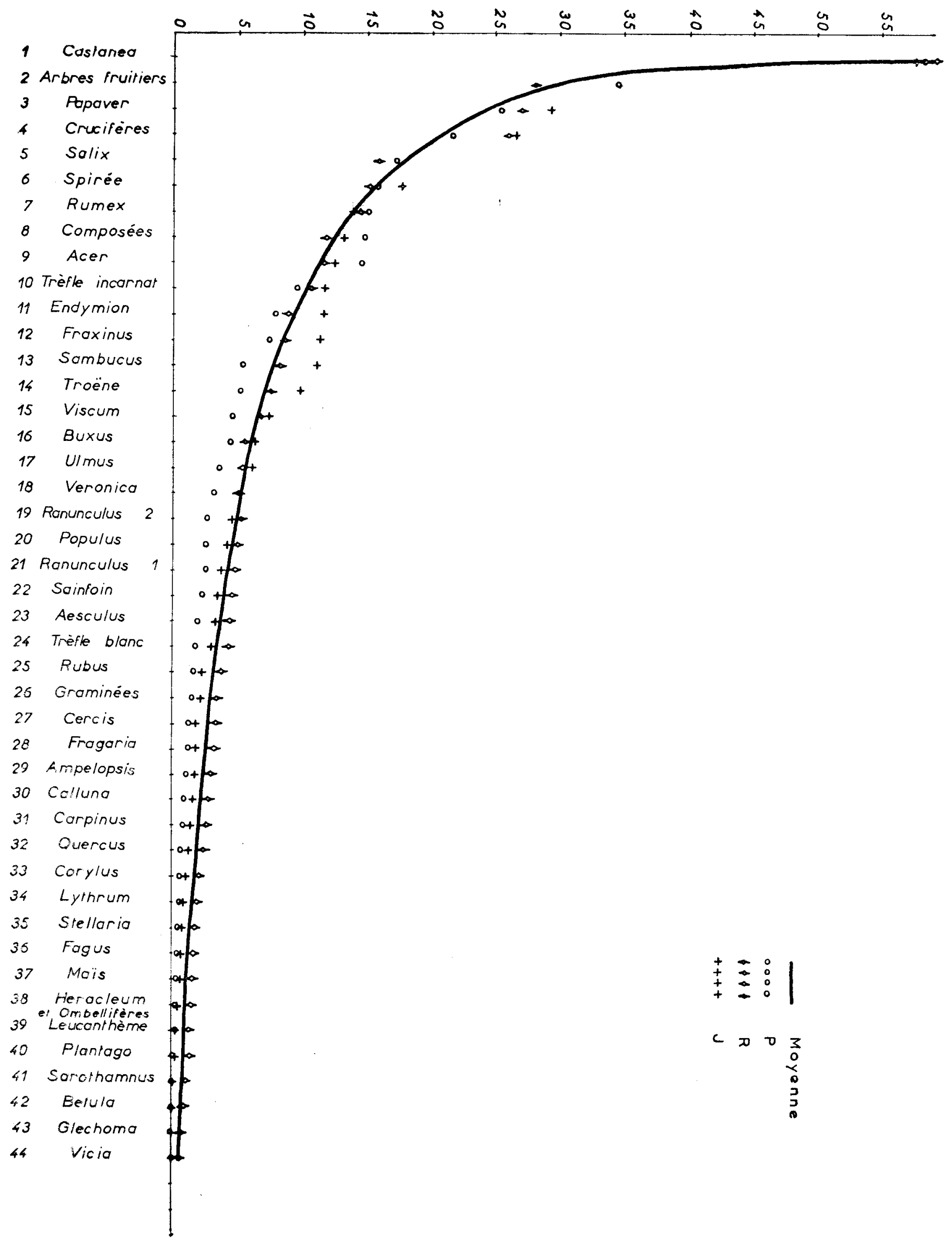

FIG. รั0. 
différences constatées et des facteurs susceptibles d'avoir une certaine importance. Nous avons étudié successivement les facteurs suivants :

-- Rémanence d'un comportement antérieur, c'est-à-dire adaptation à une flore différente de celle de la Région parisienne. L'abeille choisirait dans la flore les plantes qui étaient présentes dans son ancien biotope.

- Facteur d'ordre écologique, l'abeille visitant de préférence un m1lieu végétal déterminé : prairie, forêt, etc.

- Facteur d'ordre spatial : l'abeille choisirait un certain secteur de butinage autour du rucher.

- Facteur d'ordre nutritionnel, 1'abeille butinant en fonction des besoins nutritifs de la colonie.

Examinons successivement ces facteurs et recherchons leur importance particulière.

Io Rémanence d'un comportement antérieur. - Si nous admettons une adaptation des colonies à un certain type de flore nous sommes en droit de supposer qu'après un déplacement important les abeilles feront dans la flore locale nouvelle mise à leur disposition une sélection leur permettant de retrouver au moins partiellement la flore antérieure et ceci tant que l'acclimatation ne sera pas achevée. Cette supposition implique que l'abeille soit capable de retrouver dans une flore donnée des plantes qu'elle n'a jamais visitées elle-même mais qui étaient à la base de l'alimentation de la colonie depuis de nombreuses générations dans le lieu d'origine. Nous aurions par exemple des abeilles du Romarin ou des abeilles de la Bruyère, ces plantes étant sélectivement recherchées par suite d'un mécanisme inscrit dans le comportement inné de l'insecte.

Cette hypothèse est assez séduisante et nous avons minutieusement cherché à trouver parmi les données que nous possédons les éléments nécessaires pour lui donner corps.

Examinons par exemple le comportement des ruches du groupe P, d'origine strictement méditerranéenne. Si notre hypothèse était exacte nous devrions constater chez les abeilles de ce groupe une préférence marquée pour des plantes telles que Buxus sempervirens, qui jouent dans la zone méditerranéenne un rôle plus important que dans le nord de la France. Or, le groupe $\mathrm{P}$ s'intéresse peu à Buxus mais au contraire beaucoup à des plantes telles que le Sainfoin, le Colza, la Moutarde, le Trèfle incarnat moins fréquentes dans le Midi méditerranéen que dans notre région. Acer pseudo-platanus, plante de montagne, n'est pas spécialement recherchée par les ruches du groupe J.

L.es ruches du groupe $\mathrm{R}$ provenant de l'Indre, région typiquement productrice de Sainfoin, sont peu intéressées par le Sainfoin à Buressur-Yvette.

Nous trouvons bien quelques exemples qui vont dans le sens de notre hypothèse mais ils sont rares et assez peu significatifs. L,es ruches du 
groupe J (Jura) sont les seules à s'intéresser au Crocus, plante importante dans leur région d'origine. I,es ruches du groupe $\mathrm{P}$ visitent beaucoup Cercis silicastrum plante méridionale.

Même en tenant compte des exemples favorables à l'hypothèse d'une rémanence il ne semble pas que l'on puisse sérieusement s'arrêter à cette théorie qui ne trouve pas de points d'appui solides. Le choix des pollens récoltés ne se fait donc certainement pas en fonction d'une mémoire de la flore antérieurement utilisée. Par contre il est possible que la dispersion sur un nombre plus ou moins grand d'espèces soit en rapport avec un comportement antérieur. En effet, il est possible que la flore de l'Indre soit plus riche en espèces intéressantes pour l'abeille que celle des Bouchesdu-Rhône ou du Jura. Mais rien ne nous permet d'être vraiment affirmatifs sur ce point qui demanderait pour être vérifié une étude très serrée sur place.

$2^{\circ}$ Facteur écologique. - I,hỵpothèse de l'existence d'une abeille inféodée à une flore déterminée ne pouvant être retenue, nous pouvons supposer que le choix des abeilles se fait sur une base écologique. Nous aurions alors une abeille de la forêt butinant en hauteur sur la cîme des arbres, une abeille travaillant de préférence à ras de terre sur plantes basses, etc.

Pour vérifier cette hypothèse classons les plantes visitées d'après les strates auxquelles elles appartiennent et voyons comment se comportent les différents groupes vis-à-vis de la flore ainsi classée.

Nous avons classé dans le tableau XIII les plantes visitées par les abeilles d'après leur hauteur moyenne en trois catégories :

a) La strate arborescente comprenant tous les grands arbres.

b) La strate arbustive comprenant les arbustes et arbrisseaux.

c) La strate des chamaephytes, hémicryptophytes, cryptophytes et thérophytes.

Si nous plaçons en regard l'ordre décroissant des pourcentages de pollen de ces plantes dans les récoltes des différents groupes (valeurs des moyennes pondérées tirées du tableau XII), nous constatons que l'ordre dans lequel se placent les différents groupes est à petı près quelconque. Nous ne trouvons donc pas de groupes de ruches se plaçant toujours au premier rang pour la récolte d'un ensemble de pollens provenant de plantes ayant un caractère écologique commun. Un facteur écologique lié à la hauteur des floraisons est donc à exclure.

$3^{\circ}$ Facteur spatial. - Une étude d'un facteur spatial s'avère particulièrement délicate si on veut la généraliser. Èn effet, nous n'avons pas, dans le biotope des environs du laboratoire, des aires bien délimitées présentant une flore strictement définie. De nombreux secteurs sont inaccessibles étant constitués par des propriétés privées; tout au plus peut-on définir pour certaines plantes des secteurs privilégiés oì nous les trouverons avec une plus grande densité qu'ailleurs. 


\section{TABLEAU XIII}

\begin{tabular}{|c|c|c|c|c|c|}
\hline \multirow{2}{*}{ Glantes } & \multicolumn{5}{|c|}{$\begin{array}{c}\text { Ordre décroissant des valeurs } \\
\text { des moyennes pondérées des groupes } \\
\text { (voir tableau I } 2 \text { ) } \mathrm{M}_{\mathrm{P}} \mathrm{RR}, \mathrm{M}_{\mathrm{P}} \mathrm{JJ}, \mathrm{M}_{\mathrm{P}} \mathrm{PP} \text {, etc... }\end{array}$} \\
\hline & I & 2 & 3 & 4 & 5 \\
\hline Uhnus $\ldots \ldots \ldots \ldots \ldots \ldots \ldots \ldots$ & $\mathrm{B}$. & $\mathrm{L}$ & $J$ & R & $P$ \\
\hline Arbres fruitiers $\ldots \ldots \ldots \ldots \ldots \ldots$ & $\mathrm{P}$ & $\mathrm{B}$ & $\mathrm{J}$ & $\mathrm{R}$ & $\mathrm{L}$ \\
\hline 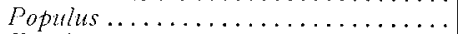 & $\mathrm{B}$ & $\mathrm{L}$ & R & 了 & $\mathrm{J}$ \\
\hline 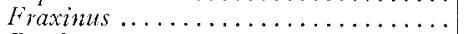 & $\mathrm{J}$ & $\mathrm{l}$. & B & $\mathrm{R}$ & $\stackrel{j}{p}$ \\
\hline Betula $\ldots \ldots \ldots \ldots \ldots \ldots \ldots \ldots \ldots$ & $\frac{1}{\mathrm{~L}}$ & $\mathrm{R}$ & $B$ & $\mathrm{~J}$ & $\mathrm{P}^{\prime}$ \\
\hline 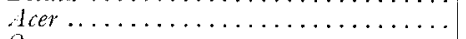 & $\mathrm{P}$ & $\mathrm{L}$ & $\mathrm{J}$ & $R$ & \\
\hline Ouercus ................... & $\mathrm{R}$ & $\mathrm{l}$ & $\mathrm{J}$, & $\mathrm{J}$ & \\
\hline 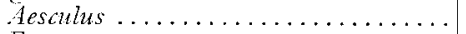 & $\mathrm{I}$ & P & $\mathrm{J}$ & $\mathrm{R}$ & \\
\hline Faguts $\ldots \ldots \ldots \ldots \ldots \ldots \ldots \ldots$ & $\mathrm{L}$ & $\mathrm{P}$ & J & $\mathrm{R}$ & \\
\hline Cercis....................... & $\mathrm{P}$ & $\mathrm{R}$ & j & & \\
\hline 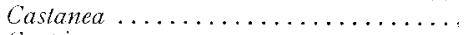 & $\mathrm{R}$ & $\mathrm{P}$ & J & & \\
\hline 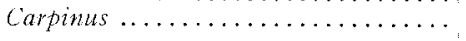 & $\mathrm{I}$ & $B$ & R & $\mathrm{J}$ & $\mathrm{P}$ \\
\hline Arbustes : & & & & & \\
\hline Salix $\ldots \ldots \ldots \ldots \ldots \ldots \ldots \ldots$ & $P$ & $\mathrm{I}$ & $\mathrm{J}$ & $\mathrm{k}$ & $\mathrm{B}$ \\
\hline 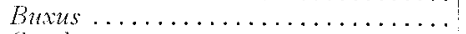 & $\mathrm{L}$ & $\mathrm{J}$ & K & $\mathrm{P}^{\prime}$ & \\
\hline Corylus $\ldots \ldots \ldots \ldots \ldots \ldots \ldots \ldots$ & R & i. & p & $\mathrm{J}$ & \\
\hline 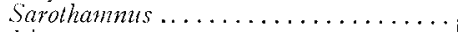 & $\mathrm{k}$ & $\mathrm{l}$ & $\mathrm{J}$ & $\stackrel{3}{P}$ & \\
\hline Ligustmum $\ldots \ldots \ldots \ldots \ldots \ldots \ldots$ & $\mathrm{I}$ & $\mathrm{R}$ & (3) & $\mathrm{P}$ & \\
\hline 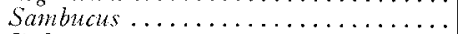 & $\mathrm{R}$ & $\mathrm{P}$ & 'j & & \\
\hline 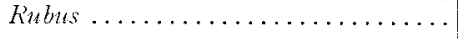 & $\mathrm{R}$ & $P$ & j & & \\
\hline Plantes basses et très basses : & & & & & \\
\hline Vicia $\ldots \ldots \ldots \ldots \ldots \ldots \ldots \ldots \ldots$ & J & $\mathrm{R}$ & l' & & \\
\hline Tritolinm repens ............... & J & $\mathrm{R}$ & P & & \\
\hline Veronica .................... & lik & $\mathrm{H}$ & $\mathrm{I}$ & $\mathrm{J}$ & $\mathrm{P}$ \\
\hline Kanunculus I ................ & $\mathrm{B}$ & $\mathrm{R}$ & J. & $\mathrm{J}^{\mathrm{J}}$ & $\mathrm{P}^{2}$ \\
\hline Ranunculus II ................. & $R$ & $\mathrm{~L}$ & $\mathrm{~J}$ & Pr & \\
\hline Stellaria.......................... & $\mathrm{B}$ & $\mathrm{R}$ & $\mathrm{J}$ & $\mathrm{J}$ & $P^{\prime}$ \\
\hline Endymion ................. & J & $\mathrm{L}$ & $P^{3}$ & $\mathrm{k}$ & $B$ \\
\hline 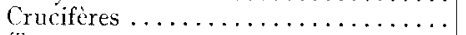 & $\mathrm{J}$ & $\mathrm{P}$ & $\mathrm{R}$ & $\mathrm{L}$ & \\
\hline Taraxacum $\ldots \ldots \ldots \ldots \ldots \ldots \ldots \ldots$ & K & B & $\mathrm{J}$ & J & J" \\
\hline Glechoma $\ldots \ldots \ldots \ldots \ldots \ldots \ldots \ldots$ & $\mathrm{R}$ & J3 & $\mathrm{I}$, & 光 & $\mathrm{P}$ \\
\hline Graminées................ & $\mathrm{R}$ & J & $\mathrm{P}^{\prime}$ & & \\
\hline Fragaria............. & $\mathrm{J}$ & $\mathrm{R}$ & $\mathrm{p}$ & & \\
\hline Trifolium incamalum ............. & ly & $\mathrm{J}$ & $\mathrm{L}$ & $\mathrm{R}$ & \\
\hline 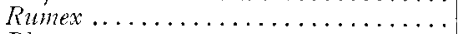 & $\mathrm{R}$ & $j$ & $\mathrm{P}$ & & \\
\hline Plantago ........... & L. & $\mathrm{k}$ & $\mathrm{P}$ & $\mathrm{J}$ & \\
\hline Sainfoin. ......... & $\mathrm{J}$ & $\mathrm{P}$ & $\mathrm{R}$ & & \\
\hline Leucanthemum $\ldots \ldots \ldots \ldots \ldots \ldots$ & R & $\mathrm{J}$ & $\mathrm{P}$ & & \\
\hline Papaver ............ & $\mathrm{J}$ & $\mathrm{R}$ & $P$ & & \\
\hline Composées diverses .. & $\ddot{\mathrm{P}}$ & $\mathrm{J}$ & $\mathrm{R}$ & & \\
\hline 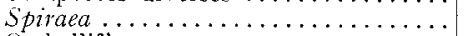 & $\mathrm{P}$ & $\mathrm{R}$ & $\mathrm{J}$ & & \\
\hline Ombellifères ..................... & $\mathrm{R}$ & $\mathrm{P}$ & J & & \\
\hline
\end{tabular}

Examinons en détail deux secteurs très différents par leur flore et qui représentent pour l'abeille partant du laboratoire le choix entre deux directions opposées : les bords humides de l'Yvette d'une part, la forêt bordant le plateau d'autre part (voir carte fig. 7). Si nous ne retenons que les plantes les plus caractéristiques et qui ne peuvent guère être représentées à la fois dans ces deux secteurs nous pouvons, comme dans l'hypothèse d'un facteur écologique établir deux listes de plantes et comparer ces listes à l'ordre d'importance des moyennes pondérées des différents groupes (valeurs du tableau XII).

Il ne se dégage rien de net du tableau XIV. Les plantes d'un même secteur ne sont pas visitées d'une façon uniforme par les abeilles des 
TABLEAU XIV

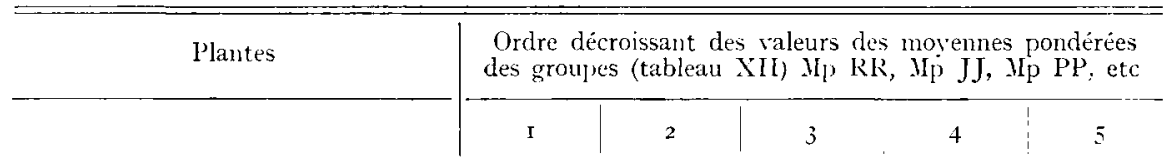

Vallée de l'Yvette (plantes du bord des caux)

\begin{tabular}{|c|c|c|c|c|}
\hline 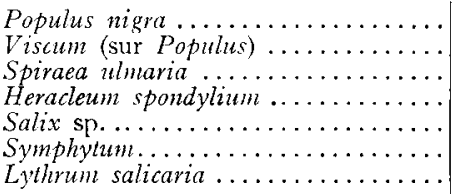 & $\begin{array}{l}\mathrm{B} \\
\mathrm{J} \\
\mathrm{H} \\
\mathrm{R} \\
\mathrm{I} \\
\mathrm{I}\end{array}$ & $\begin{array}{l}\mathrm{I}, \\
\mathrm{I}^{\prime} \\
\mathrm{R} \\
\mathrm{I} \\
\mathrm{I} \\
\mathrm{P} \\
\mathrm{P}\end{array}$ & R & $\begin{array}{l}\text { l' } \\
\mathrm{L}\end{array}$ \\
\hline
\end{tabular}

Foret et terrains voisius laissés en friche.

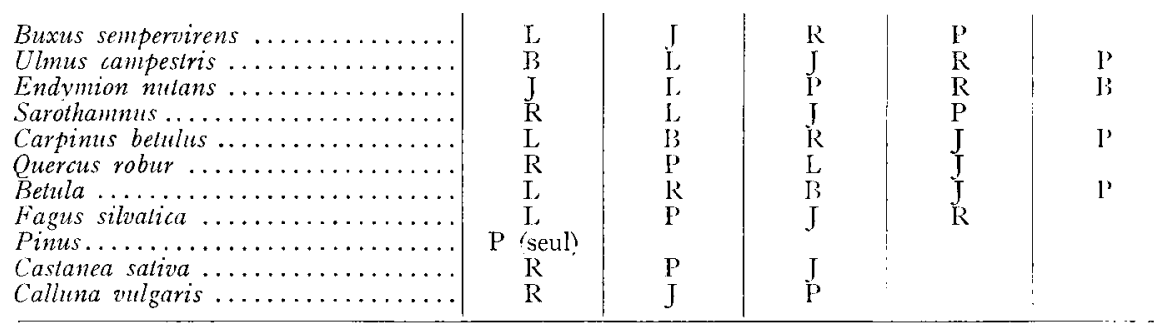

différents groupes. Le fait est particulièrement net pour Populus et Viscum. Le Gui parasite surtout les Peupliers noirs du bord de 1'Yvette. La floraison des deux plantes se fait à un intervalle de temps très court. S'il existait des secteurs de butinage bien définis on devrait voir les abeilles d'un même groupe se polariser de façon sensiblement parallèle sur le Gui et sur le Peuplier noir. Or il n'en est rien ; nous constatons même que le groupe $J$ qui occupe le premier rang pour le Gui est celui qui visite le moins le Peuplier noir.

I1 est certain, comme l'a montré LEComte (I956) que les abeilles suivent des chemins privilégiés et ne partent pas au hasard à la recherche de leur nourriture. Mais il ne suffit pas probablement qu'une plante se trouve dans une direction privilégiée de butinage pour que les abeilles s'y intéressent spécialement. Les choses ne sont pas aussi simples et d'autres facteurs doivent intervenir pour la fixation des butineuses sur les sources de nourriture.

$4^{\text {o }}$ Facteur nutritionnel. - N'ayant rien trouvé de positif du côté des facteurs écologiques ou spaciaux nous avons cherché dans une autre direction qui est celle d'un facteur mutritionnel. Nous avons pris pour hypothèse de base que les butineuses opèrent un choix parmi les pollens qui se trouvent à leur disposition dans la nature en fonction de leur valeur nutritive ou, tout au moins en fonction de certaines propriétés biologiques liées à la composition chimique du pollen. On sait déjà depuis les travaux de Maurizio (I954) que tous les pollens n'ont pas le même intérêt pourr la colonie. Certains d'entre eux ont une action marquée sur des organes 
tels que les ovaires, le corps adipeux, les glandes pharyngiennes ; ce sont des pollens doués d'une valeur physiologique élevée. D'autres pollens sont beaucoup moins actifs sur le plan physiologique. On pourait donc supposer que le choix des abeilles se porte sur les différentes plantes en fonction de la valeur nutritive du pollen qu'elles fournissent. Le travail que nous avons fait dans cette direction représente un développement assez long pour que nous lui consacrions un chapitre entier; les conclusions que l'on peut en tirer sont importantes et nous pouvons indiquer dès maintenant qu'elles sont de nature à nous faire placer au premier rang des facteurs agissant sur le choix des pollens leur valeur nutritive.

\section{CHAPITRF, V}

\section{RAPPORTS ENTHE LA VARIATION QUALITATIVE ET LA NUTRITION}

Pour pouvoir comparer facilement les différentes récoltes de pollen quant à leur valeur nutritive nous avions besoin d'un test simple et maniable, tout au moins dans un prenier stade de l'expérimentation. Il ne pouvait en effet, être question de tester biologiquement sur abeilles encagées les centaines d'échantillons dont nous avions à connaître la valeur nutritive. Ces tests biologiques, tels qu'ils ont été pratiqués par MaUrizio, PAIN, DE GrooT, etc. ne renseignent d'ailleurs que sur l'activité physiologique à l'égard des glandes pharyngiennes, des ovaires et des corps adipeux. Il n'est pas certain que leur valeur alimentaire pour les larves soit liée rigoureusement aux constituants qui agissent sur l'abeille adulte. Nous aurions risqué en ne tenant compte que de l'activité physiologique sur l'abeille adulte de négliger un aspect important dı problème, celui de la nutrition de la larve. Nous avons donc adopté un test, non pas biologique trop difficile à manier, mais chimique, celui de la teneur en azote total.

La teneur en azote total constitue, non pas le seul élément intéressant pour juger de la valeur nutritive du pollen mais sans aucun doute l'un des principaux. Le pollen constitue en effet la seule source d'azote assimilable de la ruche et, en dosant l'azote dans le pollen nous avions quelques chances de déceler des liaisons importantes entre ce facteur et ce que nous savons déjà de la récolte du pollen.

\section{A. - MÉTHODE}

Pour doser l'azote total dans le pollen nous avons utilisé la méthode du microdosage de Kjeldahl. Dans un matras de 50 cc on pèse environ $200 \mathrm{mg}$ de pollen sommairement séché. On ajoute $3 \mathrm{cc}$ d'acide 
sulfurique et une trace de catalyseur au sélénium ( $\mathrm{S} \mathrm{O}_{4} \mathrm{Cu}$ : I partie, $\$ \mathrm{SO}_{4} \mathrm{~K}_{2}$ : I partie, Se : 0,2 partie). On minéralise avec précaution car les produits moussants sont assez gênants. Dans un appareil classique pour le dosage de l'azote on neutralise l'acide sulfurique à la soude concentrée; on entraîne l'ammoniac à la vapeur d'eau; on recueille l'ammoniac sur une solution d'acide borique à $20 \mathrm{~g}$ par litre et on dose en retour par une solution titrée d'HClN/20. I,indicateur coloré utilisé est le rouge de méthyl. On fait le poids sec du pollen analysé par passage à l'étuve à $75^{\circ}$ pendant 24 h et on corrige ensuite le pourcentage d'azote obtenu pour le ramener à un pourcentage exprimé en fonction du poids sec.

\section{B. - VARIATION DU TAUX DAZOTE DANS LE POLLEN RÉCOLTÉ PAR LES ABEILLES}

Dans une première série de dosages portant sur des échantillons de la récolte totale de certaines ruches pendant des périodes de 24 heures nous avons mis en évidence une assez grande variabilité du taux d'azote dans les récoltes selon les ruches et selon le moment de l'année. I es dosages portaient sur des récoltes de l'année I95.3 (ruches de la série B). Voici quelques-uns des résultats obtenus.

'TABliat XV'

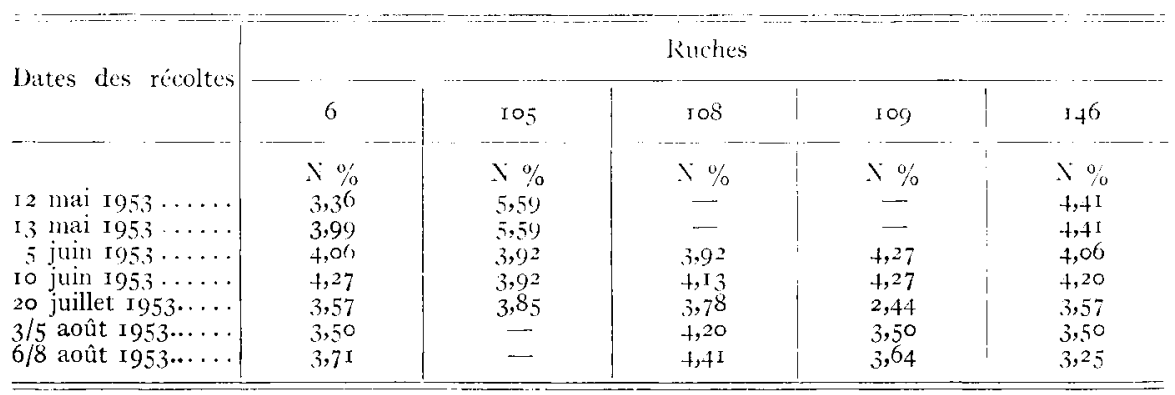

En mai r 953 des dosages d'azote effectués dans les récoltes de différents ruchers autour du laboratoire dans un rayon de $30 \mathrm{~km}$ environ ont donné les résultats suivants :

\begin{tabular}{|c|c|}
\hline Choisel & $3,85 \%$ \\
\hline Rambouillet .. &,$+ 06 \%$ \\
\hline Saint-Aubin I. . &,$+ 27 \%$ \\
\hline Saint-Aubin II. & $\therefore, 99 \%$ \\
\hline La Jinière $\ldots \ldots \ldots \ldots \ldots \ldots \ldots$ & $4,27 \%$ \\
\hline La Martinière. . . . . . . . . . . . . & $3,09 \%$ \\
\hline Bures ...................... & $3,25 \%$ \\
\hline
\end{tabular}

Ces premiers résultats montraient que le taux d'azote dans les récoltes de pollen présente une grande variabilité $(2,44 \mathrm{p}$. Ioo à $5,59 \mathrm{p}$. Ioo sur 70 échantillons analysés environ) selon les ruches, selon les époques et selon les localités. En appliquant le coefficient 6,25 au taux d'azote 
pour évaluer la teneur en protéines brutes on trouve donc une variation de 1.5,2:j à $34,9.3$ p. 100 dans le taux des protides, ce qui est considérable.

Aucune loi ne pouvait évidenment se dégager de ces premières expériences qui ne reposaient que sur un sondage rapide.

Nous savons déjà par l'étude de la bibliographie que le taux d'azote du pollen est susceptible de variations importantes; il n'y avait donc pas lieu d'être surpris de constater des variations notables mais il nous apparut important d'étudier systématiquement de quelle façon se fait cette variation car nous avions déjà cru trouver quelques directions intéressantes dans les premiers chiffres obtenus.

I,es essais furent repris en 19:5; et cette fois toutes les récoltes furent systématiquement soumises à l'analyse en vue de doser l'azote. Pour chaque ruche un dosage d'azote fut fait pour chaque semaine de façon à pouvoir établir une courbe de la variation du taux de ce corps. Par ailleurs on procéda au calcul de l'azote total représenté par la nuasse de pollen récoltée en multipliant pour chaque semaine et pour chaque ruche la quantité de pollen par le pourcentage d'azote.

I es figures 5 I à 54 expriment la variation du taux d'azote dans les récoltes au cours de l'année I955 dans nos différentes ruches expérimentales. Pour plus de clarté nous arons regroupé sur la figure 5.5 les moyennes du taux d'azote dans les différents groupes. Sur cette même figure nous avons fait mention des floraisons les plus importantes et qui sont responsables des fluctuations du taux d'azote.

I a lecture de ce dernier graphique est particulièrement intéressante car elle met clairement en évidence une variation annuelle du taux d'azote des récoltes, variation qui est imposée par le milieu extérieur, c'est-à-dire par la flore, et qui intéresse toutes les colonies. Les premières floraisons pu printemps ne fournissent que des pollens pawrres en azote; avec l'apparition du saule et ensuite des arbres fruitiers la teneur en azote atteint un maximum en mai. Après le mois de mai la teneur en azote est en baisse constante avec des variations momentanées telles que celles qu'on observe

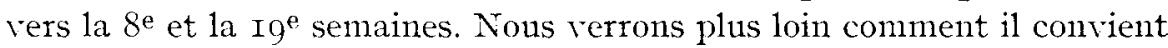
d'interpréter les divers accidents constatés de mai à octobre et qui apportent des modifications importantes au taux d'azote.

Nous constaterons encore que le taux d'azote dans les récoltes varie selon les colonies. Lies ruches du groupe $\mathrm{P}$ présentent un taux très élevé jusqu'en juillet et moyen à partir de ce mois. Celles du groupe J ont tout d'abord des taux inférieurs à celles du groupe $P$ puis elles s'alignent sensiblement sur ce groupe et sonvent le dépassent. Quant aux ruches du groupe $\mathrm{R}$, le taux d'azote de leurs récoltes est presque toujours plus faible que celui des autres groupes sauf en juillet oì elles passent

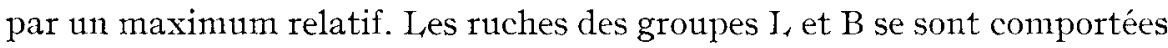




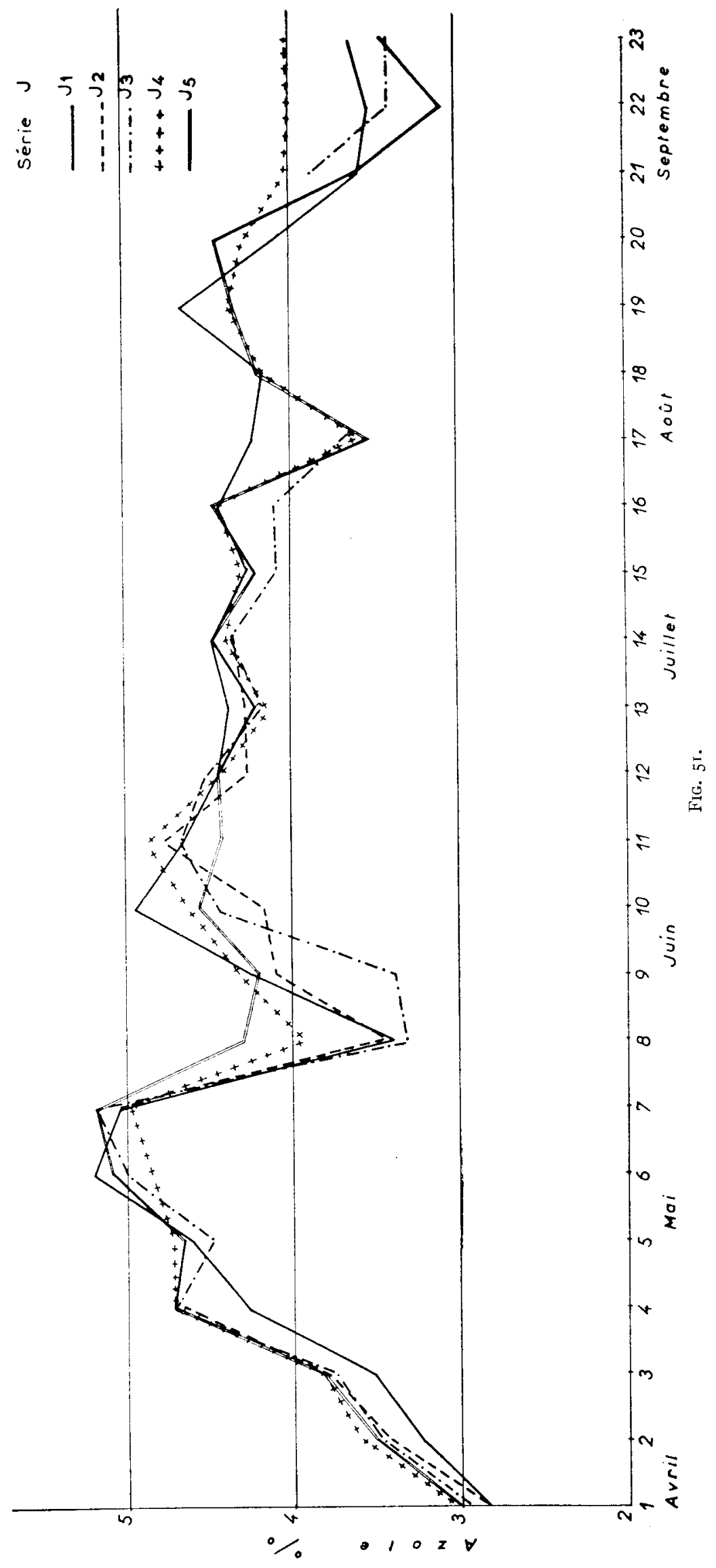




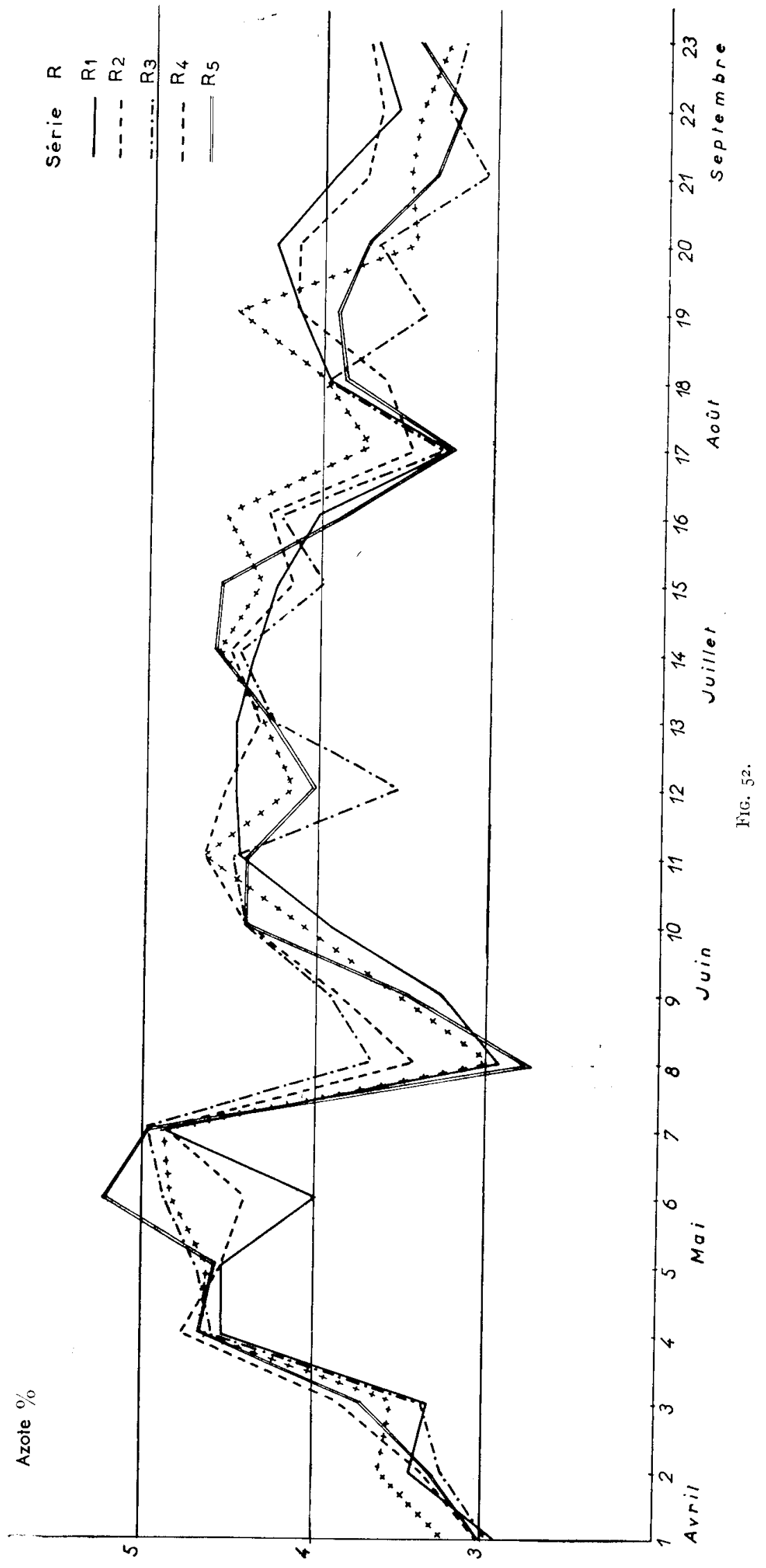




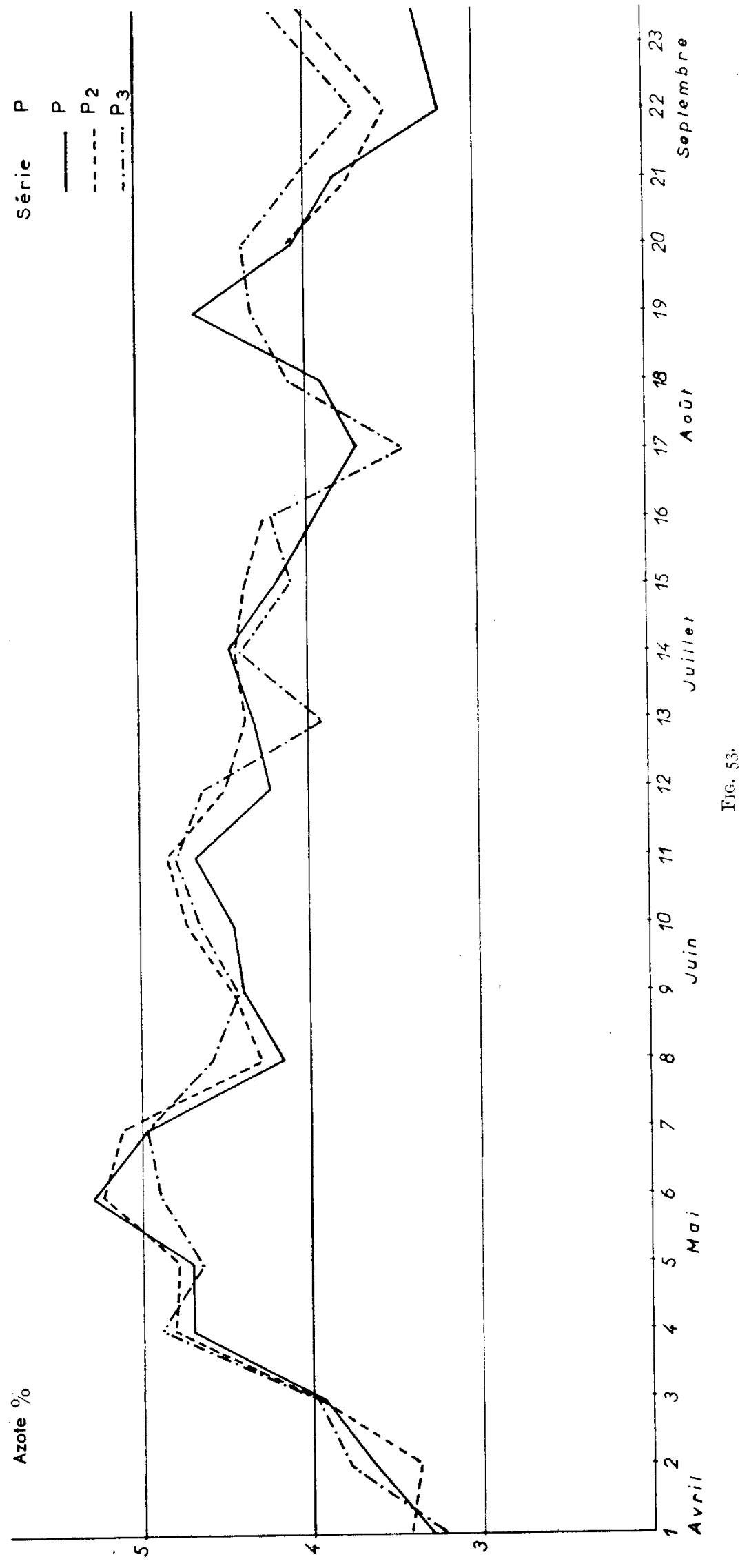




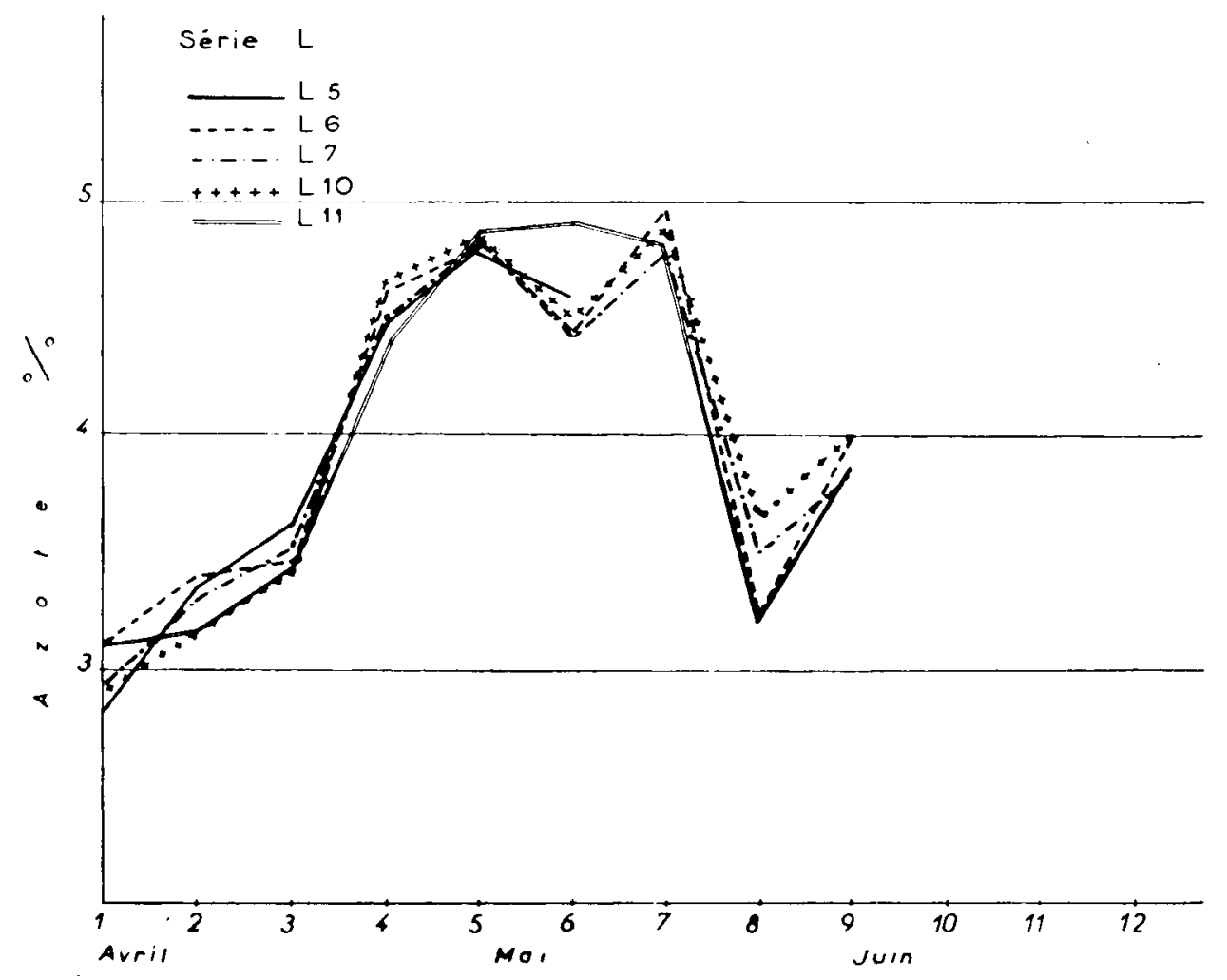

I'IS. 54.

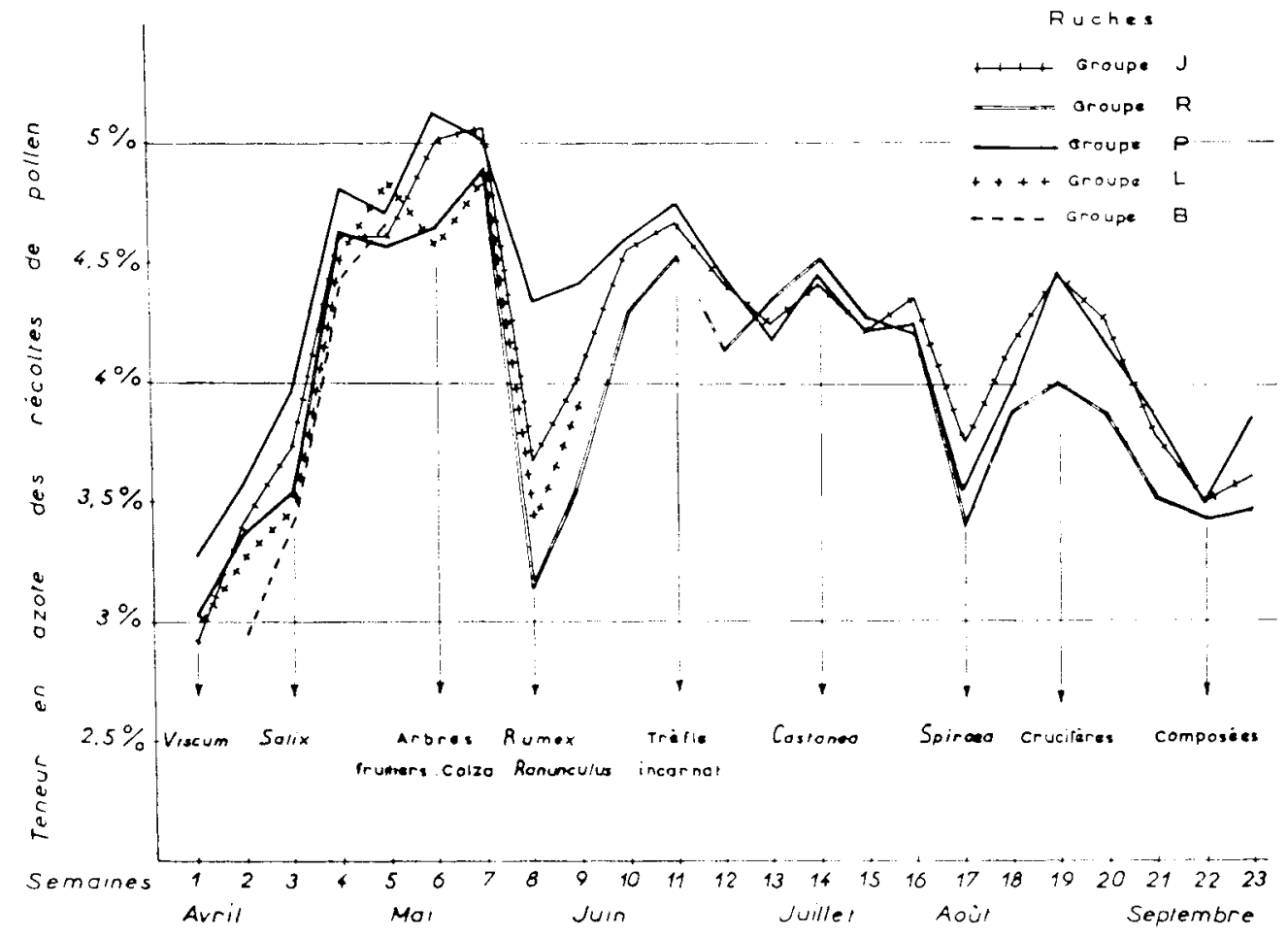

Frg. 55. 
pendant la période où elles ont été observées sensiblement comme les ruches du groupe $R$.

Devant ces résultats fort importants il nous a paru utile de procéder à certains contrôles. Il fallait tout d'abord vérifier les résultats en procédant à l'analyse d'échantillons de pollen purs, soigneusement triés dans les récoltes, de façon à connaître les pollens responsables des variations enregistrées.

Nous avons donc repris dans tout le matériel disponible des échantillons susceptibles de nous fournir des pollens purs en quantité suffisante. Les résultats figurent au tableau XVI.

On remarquera tout d'abord que chaque fois qu'il nous a été possible de faire deux dosages successifs sur un même échantillon nous avons obtenu une précision satisfaisante puisque les résultats sont acquis à \pm o, Io p. Ioo dans les cas les plus défavorables et à o,o I p. roo dans les cas les plus farorables. L'erreur systématique sur les pourcentages est donc relativement faible. Par contre lorsque nous considérons deux dosages portant sur un même pollen pris dans des ruches différentes nous relevons une différence qui peut atteindre 0,3 à 0,4 p. Ioo. Cette différence peut être due au fait que le triage, bien qu'il soit fait soigneusement et vérifié au microscope, ne peut pas être parfait. Un pourcentage de 5 p. roo de pollens étrangers peut se présenter du fait des grains de pollen étrangers collés aux pelotes et du fait des mélanges opérés parfois par les abeilles dans la confection des pelotes. Fille peut aussi provenir du fait que les abeilles ont utilisé plus ou moins de nectar pour agglomérer les pelotes. Cette dernière hypothèse semble confirmée par le fait que,de deux pourcentages d'azote obtenus sur un même pollen pour une ruche des groupes $P$ ou $J$ ou pour une ruche du groupe $R$, c'est presque toujours celui du groupe $\mathrm{R}$ qui est le plus faible.

Afin d'approfondir un peu ce phénomène nous avons procédé à 1'analyse du pollen de Saule dans toutes les ruches pendant la $2^{\mathrm{e}}$ semaine de I955. Le tableau XVII donne le résultat de ces analyses.

La moyenne générale inter-groupes pour la teneur en azote du pollen de Saule ressort à 4 , or p. Ioo.

Il existe donc une certaine variabilité du taux d'azote dans les pelotes d'un même pollen provenant de ruches différentes; elle provient pour une part des mélanges inévitables avec des pollens étrangers, d'autre part d'un taux de sucre plus ou moins élevé provenant du nectar qui sert à agglomérer le pollen. Il semble bien que les ruches P et J présentent le caractère commun de mêler peu de nectar au pollen qu'elles récoltent.

Nous devrons tenir compte de ce fait dans l'avenir mais son importance pratique ne paraît pas considérable étant donnée l'ampleur des variations dues à la teneur en azote des différents pollens. 
TABLEAU XVI

\begin{tabular}{|c|c|c|c|}
\hline Pollens & Ruches & Années & Azote $\%$ \\
\hline Viscum & $\mathrm{B}$ & $53 / 54$ & $3, \mathrm{II}$ \\
\hline (n & $\mathrm{J} 3$ & $\begin{array}{c}33754 \\
55\end{array}$ & $2,91 \pm 0,10$ \\
\hline 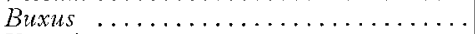 & $\mathrm{B}^{3}$ & $53 / 54$ & 3,25 \\
\hline Veronica ..................... & $\mathrm{R}_{3}$ & 55 & $3,12 \pm 0,02$ \\
\hline Ulmus $\ldots \ldots \ldots \ldots \ldots \ldots \ldots \ldots \ldots \ldots$ & $B 109$ & 55 & $3,92 \div 0,02$ \\
\hline Ranunculus $1 . \ldots \ldots \ldots \ldots \ldots \ldots$ & I) 105 & 55 & $3, \mathrm{II} \pm 0,0 \mathrm{I}$ \\
\hline Ranunculus $1 \ldots \ldots \ldots \ldots \ldots \ldots \ldots$ & $\mathrm{R}^{\mathrm{I}}$ & 55 & $3,04 \div 0,03$ \\
\hline Ranunculus $I I \ldots \ldots \ldots \ldots \ldots \ldots \ldots$ & $\mathrm{R}$ & 55 & $2,8 \mathrm{I} \div 0,04$ \\
\hline 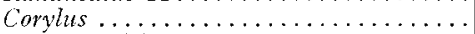 & $\mathrm{R}_{\mathrm{I}}$ & 55 & 2,53 \\
\hline Arbres fruitiers..$\ldots \ldots \ldots \ldots \ldots$ & $B$ & 54 & 4,72 \\
\hline " $\quad \ldots \ldots \ldots \ldots \ldots \ldots \ldots$ & $B$ & $53 / 54$ & 4,74 \\
\hline$\ldots \ldots \ldots \ldots \ldots \ldots$ & $I_{2}$ & 55 & $4,97 \div 0,01$ \\
\hline (Promis) $\ldots \ldots \ldots \ldots$ & $B$ & 54 & 4,56 \\
\hline (Prunus) ........... & $\mathrm{J} 3$ & 55 & $4,78 \pm 0,08$ \\
\hline Endymion ....................... & 3 & 55 & $5,39 \div 0,05$ \\
\hline 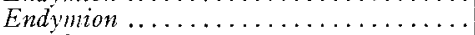 & $\mathrm{R}$ & 55 & $5,29 \pm 0,04$ \\
\hline 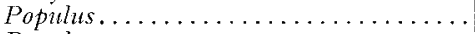 & - & 55 & $2,5 \mathrm{I}$ \\
\hline Populus ...................... & 136 & 55 & $2,92 \pm 0,03$ \\
\hline Colza $\ldots \ldots \ldots \ldots \ldots \ldots \ldots \ldots$ & $\mathrm{B}$ & 54 & 4,87 \\
\hline 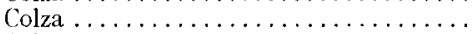 & B 6 & 55 & $4,86 \div 0,10$ \\
\hline 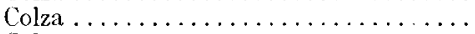 & $\mathrm{P}_{\mathrm{I}}$ & 55 & $4,9 \mathrm{I}-0,0 \mathrm{I}$ \\
\hline Colza . . . $\ldots \ldots \ldots \ldots \ldots \ldots \ldots$ & P I & 55 & $4,94 \div 0,10$ \\
\hline  & $\mathrm{J} 5$ & 55 & $4,46=0,10$ \\
\hline Cruciféres .................... & Ji & 55 & $4,30 \div 0,05$ \\
\hline 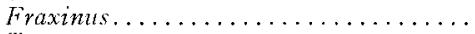 & $\mathrm{j}$ & 55 & $3,72+0,07$ \\
\hline 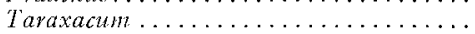 & 3 & 54 & 2,55 \\
\hline Taraxacum $\ldots \ldots \ldots \ldots \ldots \ldots \ldots$ & $\mathrm{R}_{1}$ & 55 & $2,66 \quad 0,0 \mathrm{I}$ \\
\hline 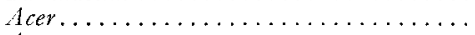 & 13 & $5.3 / 54$ & 5,04 \\
\hline 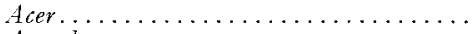 & $\mathrm{R}_{2} / \mathrm{R}_{3}$ & 55 & $5,02 \pm 0,01$ \\
\hline desculus & $\mathrm{J}_{\mathrm{I}}$ & 55 & $5,85 \pm 0,05$ \\
\hline$\ldots \ldots \ldots \ldots \ldots$ & $\mathrm{k} 2$ & 55 & $5,70 \pm 0,03$ \\
\hline 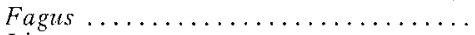 & L 5 & 55 & $2,30 \pm 0,05$ \\
\hline 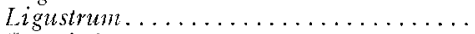 & $\mathrm{L} 7 / 1.10$ & 55 & $4,15 \div 0,05$ \\
\hline 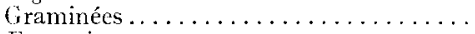 & $\mathrm{R}+$ & 55 & $3,09 \pm 0,04$ \\
\hline Fragaria $\ldots \ldots \ldots \ldots \ldots \ldots \ldots \ldots \ldots$ & $\mathrm{J} \mathrm{J} / \mathrm{J}_{4}$ & 55 & $3,49 \pm 0,01$ \\
\hline Trifolium incarnatum........... & $\mathrm{J} 2$ & 55 & $5,03 \pm 0,03$ \\
\hline Runex ............ & R 5 & 55 & $2,09 \pm 0,02$ \\
\hline Plantago......... & $\mathrm{P} 3$ & 55 & $3,67 \pm 0,07$ \\
\hline Composées $\ldots \ldots \ldots \ldots \ldots \ldots \ldots \ldots$ & R 5 & 55 & $3,01+0,02$ \\
\hline Composées....................... & $\mathrm{R} 3$ & 55 & $3,73 \pm 0,03$ \\
\hline 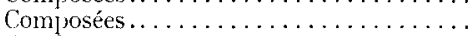 & $\mathrm{Pi}$ & 55 & $3,70 \pm 0,07$ \\
\hline 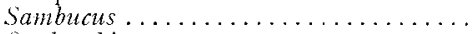 & $J$ et $R$ & 55 & $4,28 \pm 0,06$ \\
\hline Onobrychis $\ldots \ldots \ldots \ldots \ldots \ldots \ldots \ldots$ & $J+$ & 55 & $5,35+0,05$ \\
\hline Vicia...... & 13 & 53 & 5,27 \\
\hline Trifolium repens ................ & B3 & $5 3 \longdiv { 5 4 }$ & 4,50 \\
\hline Trifolium repens.................. & $j .3$ & 55 & $4,4 r-0,03$ \\
\hline Leucanthemum .................. & $\mathrm{R}_{3}$ & 54 & $2,83-0,04$ \\
\hline 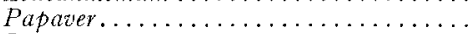 & 15 & 55 & $4,60+0,07$ \\
\hline 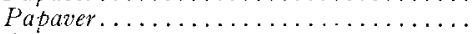 & $B$ & 55 & $4,57-1$, \\
\hline 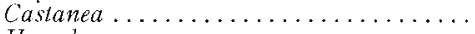 & J 1 & 55 & $4,27+0,05$ \\
\hline 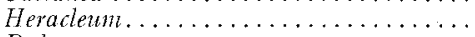 & $\mathrm{R} 1 / \mathrm{R} 2$ & 55 & $4,90 \pm 0,06$ \\
\hline 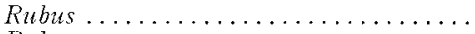 & $\mathrm{P}^{1} 2$ & 55 & $4,63 \doteqdot 0,04$ \\
\hline 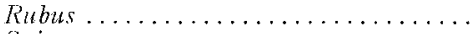 & $R_{5}$ & 55 & $4,49+0,03$ \\
\hline 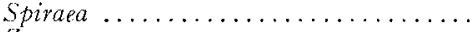 & $\mathrm{R}$ & 55 & $3,01+0,07$ \\
\hline$Z e a \ldots \ldots \ldots \ldots \ldots \ldots \ldots \ldots \ldots \ldots$ & $\mathrm{R} 3$ & 35 & 3,0 I $\quad$ o,o6 \\
\hline 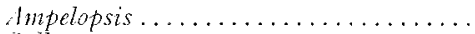 & $\mathrm{R}$ & 55 & $3,92 \div 0,01$ \\
\hline Calluna ............... & R & 55 & $4,23-i 0,0,3$ \\
\hline Polygonum fagopyrzin ............... & - & 55 & 2,60 \\
\hline 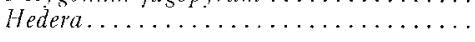 & $\mathrm{J}$ & 53 & 4,50 \\
\hline
\end{tabular}

Revenons au graphique 55 et examinons-le en tenant compte des teneurs en azote des pollens purs que nous venons de déterminer et du spectre pollinique des récoltes.

Les faibles pourcentages de la première période de l'année s'expli-

Annales des Abeilles. -1959 . 
quent par l'abondance de Viscum (3 p. roo), Veronica (3,12 p. Ioo), Buxus (3,25 p. I00), Ranunculus I (3,04 p. I00). La montée rapide correspond ensuite à l'apparition de Salix (4,0I p. Ioo) et surtout des Arbres fruitiers (4,90 p. I00). Aesculus (5,85 p. IOo) et Colza (4,94 p. IOo) contribuent encore à élever la moyenne jusqu'à la $7^{\mathrm{e}}$ semaine. La chute de 1a $8 \mathrm{e}$ semaine est due à Rumex (2,09 p. Ioo) et Ranunculus II (2,8I p. IOO).

TABLEAU XVII

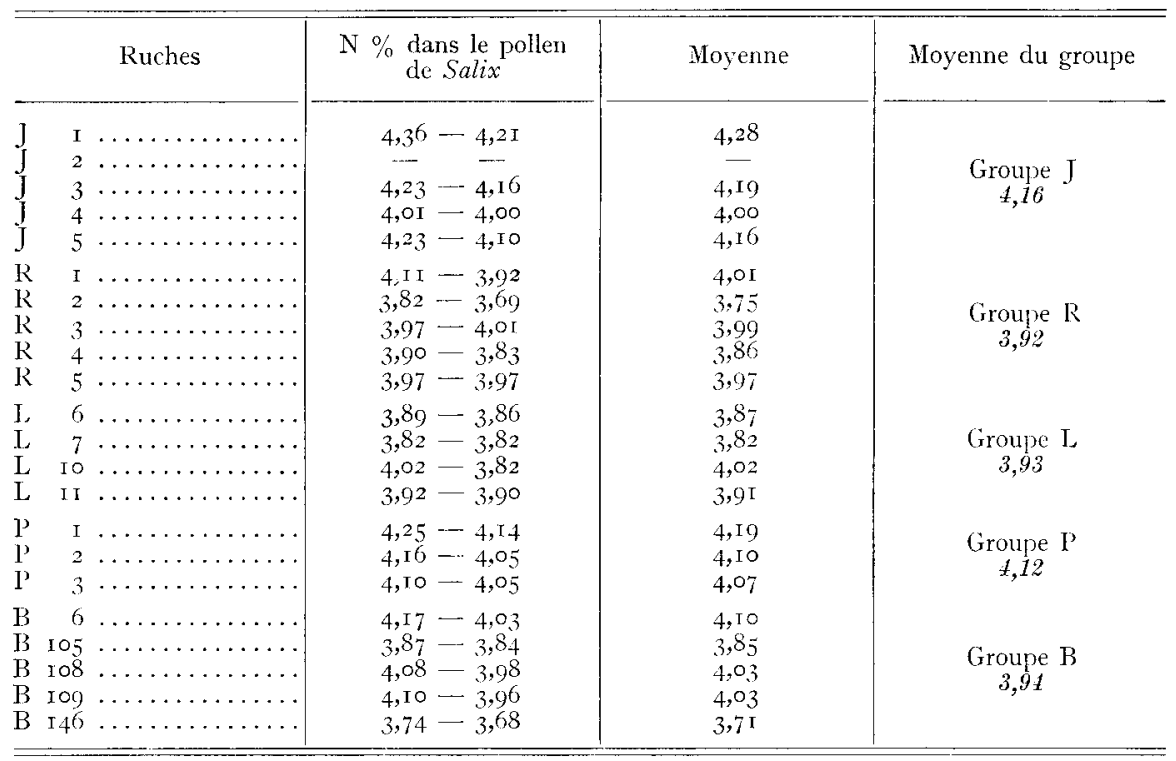

La remontée de la II $^{\mathrm{e}}$ semaine peut être attribuée à Trifolium incarnatum (5,03 p. Ioo), celle de la $\mathrm{I}_{4}^{\mathrm{e}}$ semaine à Castanea $(4,27 \mathrm{p}$. 10o) et Trifolium repens $\left(4,4 \mathrm{I} \mathrm{p}\right.$. IOO). La chute de la $\mathrm{I} 8^{\mathrm{e}}$ semaine est due à l'abondance du pollen de Spiraea (3,OI p. IOo) tandis que la remontée de la $\mathrm{I}^{\mathrm{e}}$ semaine est attribuable aux Crucifères (4,30 p. Ioo). La fin du graphique montre le mélange des Composées ( 3 à 3,70 p. Ioo) et des Crucifères.

Nous pouvons aussi expliquer les différences entre les ruches du groupe $R$ et celles du groupe $P$ par le fait que leur spectre pollinique est différent. Alors que Rumex et Ranunculus sont abondants chez les ruches $\mathrm{R}$ dans la $8^{\mathrm{e}}$ semaine ils sont plus rares dans le groupe $\mathrm{P}$. Nous allons d'ailleurs reprendre en détail cette question un peu plus loin.

En conclusion de cette étude de la variation du taux d'azote dans les récoltes de pollen nous noterons qu'il existe une variation saisonnière de ce taux, variation qui n'avait jamais été mise en évidence. Nous avons retrouvé le même phénomène en 1956 . Ayant dosé l'azote dans les récoltes 
provenant de différentes ruches en avril et mai nous avons constaté la progression suivante :

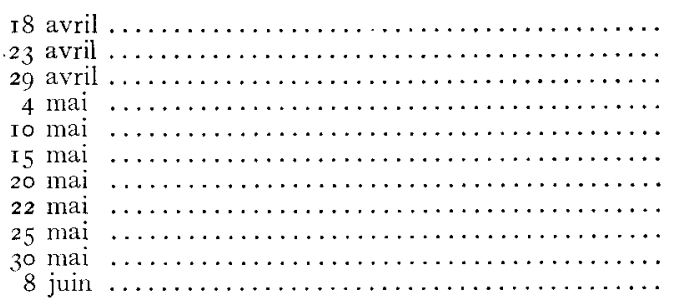

$$
\begin{aligned}
& 3,03 \% \\
& 4,42 \% \\
& 4,28 \% \\
& 4,71 \% \\
& 4,03 \% \\
& 4,42 \% \\
& 4,39 \% \\
& 4,83 \% \\
& 4,84 \% \\
& 5,41 \% \\
& 4,75 \%
\end{aligned}
$$

Il ne s'agit donc pas d'un phénomène accidentel particulier à l'année I955 mais au contraire d'une évolution qui paraît normale et constante. En I953 nous avions aussi trouvé en mai des valeurs plus fortes du pourcentage d'azote dans les récoltes que pendant les autres mois.

\section{C. - SIGNIFICATION DE LA VARIATION DU TAUX D'AZOTE DANS LES RÉCOLTES DE POLLEN}

Nous avons maintenant la possibilité de reprendre où nous l'avions laissée notre étude sur l'origine des variations qualitatives constatées dans les récoltes des différentes ruches. Ainsi que nous le signalions en terminant le chapitre IV, un facteur lié à la nutrition de la colonie semble bien présider au choix opéré par les abeilles à l'intérieur d'une flore donnée. Nous avons précédemment constaté que le taux d'azote dans les récoltes est différent selon les groupes de ruches (Expériences de I955). Voyons donc dans quelle mesure il est possible de relier le choix opéré par les abeilles dans la flore et la teneur en azote des différents pollens utilisés. Deux méthodes peuvent être appliquées.

Ire méthode. - Nous classerons tout d'abord les plantes les plus importantes en 3 catégories :

a) Les plantes pour lesquelles les ruches du groupe $\mathrm{J}$ ont marqué une préférence.

b) Les plantes pour lesquelles les ruches du groupe $\mathrm{R}$ ont marqué une préférence,

c) Ires plantes pour lesquelles les ruches du groupe P ont marqué une préférence.

Nous opérons ce classement en nous basant, comme pour les précédentes comparaisons, sur les valeurs des moyennes pondérées du tableau XII qui constituent une base très sûre.

Nous éliminerons volontairement les groupes $\mathrm{I}_{1}$ et B qui n'ont pu être suivis toute l'année et qui, de ce fait, ne peuvent nous apporter que des données par trop incomplètes. Nous verrons d'ailleurs qu'il est possible de rattacher le groupe $\mathrm{L}$ au groupe $\mathrm{R}$ quant au comportement général. 
Nous classerons les plantes dans l'ordre de leur floraison et nous distinguerons une période printanière et une période estivale (tableau XVIII).

\section{TABLEAU XVIII}

\begin{tabular}{|c|c|c|}
\hline Groupe J & Groupe R & Groupe P \\
\hline \multicolumn{3}{|c|}{ Printemps } \\
\hline $\begin{array}{l}\text { Viscum } \\
\text { Buxus } \\
\text { Ulmus } \\
\text { Endymion } \\
\text { Crucifères } \\
\text { Fraximus } \\
\text { Fragaria } \\
\text { Onobrychis } \\
\text { Vicia }\end{array}$ & $\begin{array}{l}\text { Veronica } \\
\text { Ranunculus I } \\
\text { Ramunculus II } \\
\text { Stellaria } \\
\text { Corylus } \\
\text { Populns } \\
\text { Taraxacum } \\
\text { Carpinus } \\
\text { Betula } \\
\text { Glechoma } \\
\text { Sarothamnus } \\
\text { Quercus } \\
\text { Ligustrum } \\
\text { Graminés } \\
\text { Rumex } \\
\text { Plantago } \\
\text { Leucanthemum }\end{array}$ & $\begin{array}{l}\text { Salix } \\
\text { Arbres fruitiers } \\
\text { Acer } \\
\text { Aesculus } \\
\text { Fagus } \\
\text { Trifol. incarn. } \\
\text { Cercis }\end{array}$ \\
\hline \multicolumn{3}{|c|}{ Été } \\
\hline $\begin{array}{l}\text { Trifolium repens } \\
\text { Papaver } \\
\text { Lyihrum }\end{array}$ & $\begin{array}{l}\text { Castanea } \\
\text { Sambucus } \\
\text { Rubus } \\
\text { Zea } \\
\text { Ampelopsis } \\
\text { Heracleum } \\
\text { Calluna }\end{array}$ & $\begin{array}{l}\text { Composées } \\
\text { Spiraea }\end{array}$ \\
\hline
\end{tabular}

Le nombre des plantes recherchées de préférence par les ruches du groupe $\mathrm{R}$ est plus élevé que les deux autres. Ceci est la conséquence du phénomène que nous avons déjà analysé de la plus grande dispersion des activités des ruches du groupe $\mathrm{R}$.

Nous avons porté sux les graphiques 56 à $5^{8}$, en abscisses les pollens les plus spécialement recherchés par les ruches des différents groupes en les ordonnant en fonction de la valeur des moyennes pondérées calculées et en commençant par les plus fortes. Ces valeurs sont celles du tableau XII qui continue à nous servir de base.

La figure 56 représente la courbe obtenue (trait plein) lorsqu'on ordonne dans l'ordre décroissant ies pourcentages maxima atteints par les différents pollens dans le groupe J (Mp JJ (X), Mp JJ (Y), Mp JJ (Z) etc.). I,es points se rapportant aux valeurs correspondantes des groupes $\mathrm{P}$ et $\mathrm{R}$ (Mp PP (X), Mp PP (X), Mp RR (X), Mp RR (Y) etc...) se situent très au-dessous. Si nous portons sur un autre axe d'ordonnées la teneur en azote des pollens considérés nous constatons qu'ils sont tous compris à très peu de chose près entre 3 et 5 p. Ioo. Il s'agit donc, dans l'ensemble, de pollens riches en azote. Ise taux d'azote du pollen de Lythrum nous 
manque malheureusement pour compléter le graphique mais il s'agit d'une plante peu importante et qu'on peut éventuellement négliger.

I a figure 57 est établie de la même façon que la précédente mais cette fois nous ordonnons le graphique par rapport aux valeurs décroissantes des moyennes pondérées du groupe $\mathrm{P}(\mathrm{MpP}(\mathrm{X}), \operatorname{Mp} \mathrm{PP}(\mathrm{Y})$ etc...). La figure comporte deux parties différentes, l'une où sont portés les pol-

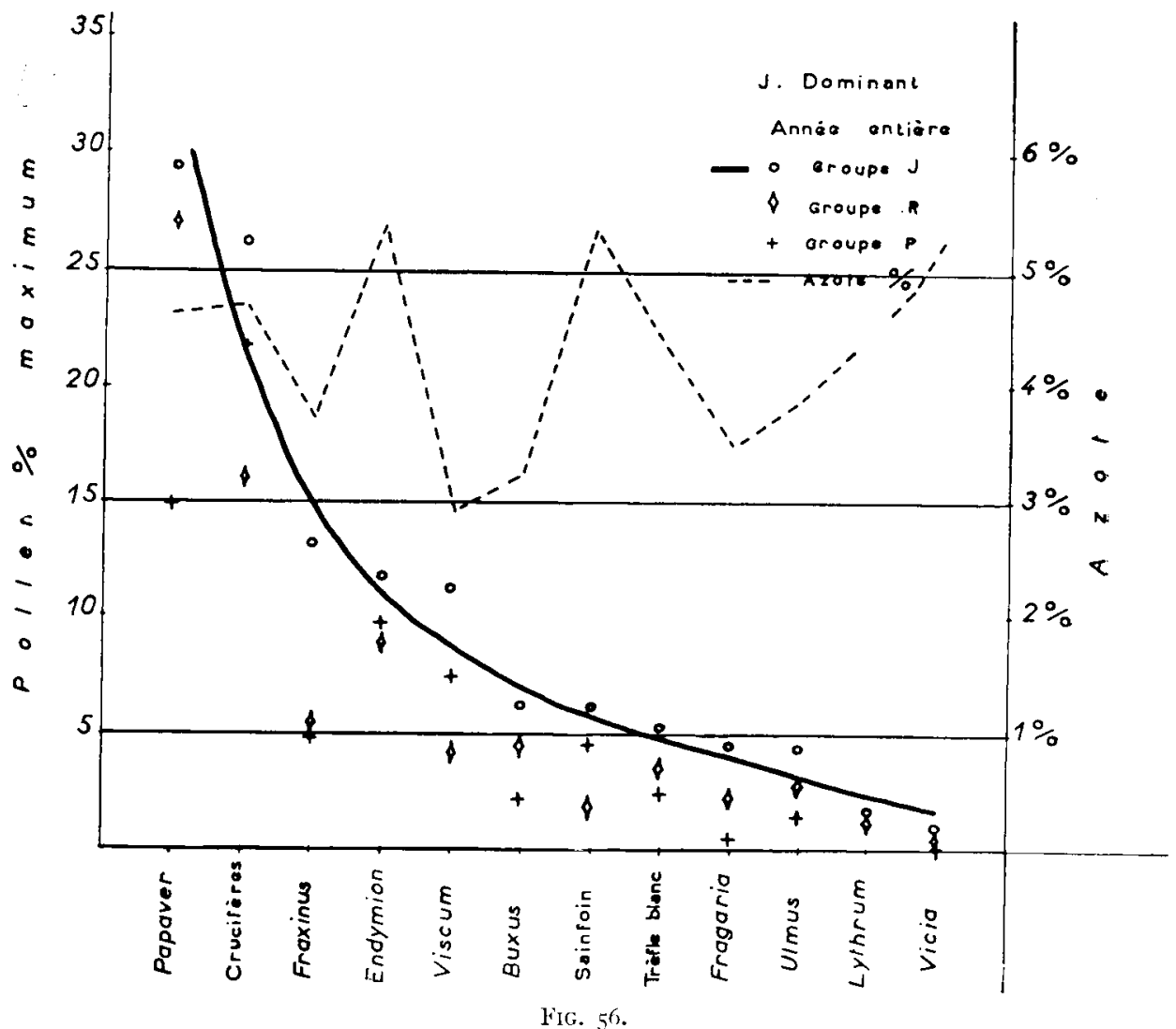

lens de printemps, l'autre où sont portés les pollens d'été. Comme dans la figure précédente, la courbe du groupe $\mathrm{P}$ se situe très au-dessus des deux autres. Nous avons cette fois une pente beaucoup plus forte car les plantes sont peu nombreuses. Si nous portons en seconde ordonnée le pourcentage d'azote des pollens considérés (trait discontinu) nous constatons qu'ils sont tous riches en azote (entre 4 et 6 p. Ioo). Seul Fagus qui ne joue qu'un rôle très secondaire ( $\mathrm{I}$ p. roo des récoltes) apporte une discordance négligeable. Par contre pour les pollens récoltés en été les teneurs en azote sont inférieures à 4 p. roo. Nous reviendrons plus loin sur cette particularité qui mérite une attention spéciale.

En fin, la figure 58 est établie, toujours sur le même principe, en fonc- 
tion des maxima du groupe $\mathrm{R}$. Comme pour le groupe $\mathrm{P}$ il convient de séparer deux périodes, l'une printanière, l'autre estivale. Dans la période printanière les ruches du groupe $\mathrm{R}$ présentent leurs maxima d'activité sur des plantes dont le pollen a un taux d'azote compris entre 2 et $4 \mathrm{p}$. roo. Quelques valeurs nous manquent mais elles correspondent à des plantes

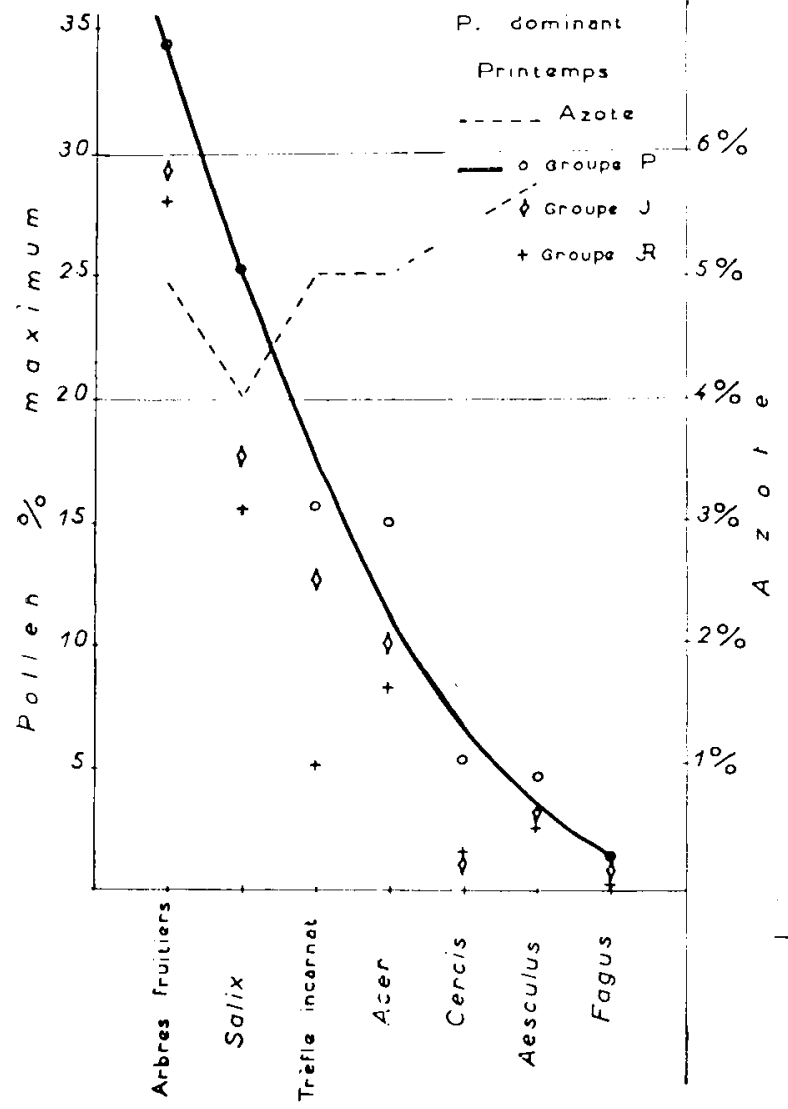

FIG. 57 .

peu importantes. Par analogie nous sommes d'ailleurs fondé à croire que ni Quercus, ni Betula ni Carpinus ne fournissent des pollens riches en azote ; ce sont en effet, botaniquement parlant, des plantes voisines de Corylus et Fagus dont le pollen est pauvre en azote.

Dans la période estivale les pourcentages d'azote sont plus élevés et s'échelonnent entre 3 et 5 p. Ioo.

Notons encore que sur la figure 58 , à la courbe ordonnée par rapport au groupe $\mathrm{R}$ correspondrait une courbe du groupe $\mathrm{P}$ qui serait presque inversée, ce qui montre clairement l'antagonisme entre les deux groupes. Là où $\mathrm{R}$ présente des maxima de récolte (Rumex, Veronica, Ranunculus) 
le groupe $P$ présente des valeurs très faibles alors que le groupe J présenterait seulement un écart important mais non une courbe inversée.

En ce qui concerne le groupe $I_{1}$ et le groupe B que nous avons négligés dans cette étude nous remarquerons simplement que les données dont nous disposons sont trop peu nombreuses pour donner des résultats intéressants. Les valeurs des pourcentages du groupe $\mathrm{L}$, sont souvent voisines

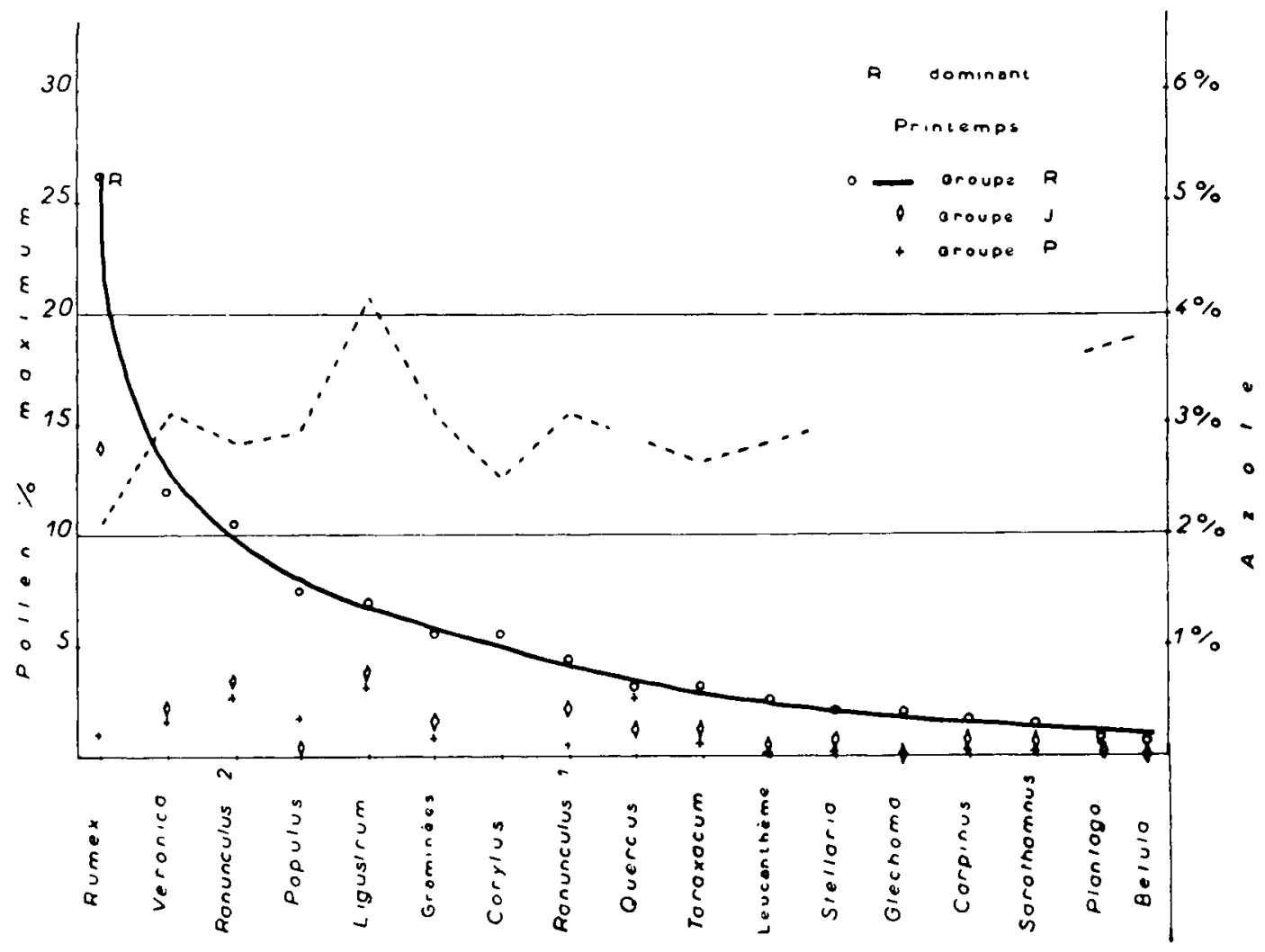

Fig. 58 .

de celles du groupe $\mathrm{R}$ mais nous ne pouvons pas nous prononcer ent toute certitude.

$2^{\mathrm{e}}$ méthode. - Nous pouvons encore rendre compte d'une façon différente des résultats obtenus en opérant de la même façon que lorsque nous avons voulu rechercher une liaison entre le biotope et le choix des pollens. Si nous classons les pollens d'après leur teneur en azote en distinguant les floraisons printanières et les floraisons estivales nous obtenons une liste en regard de laquelle nous pouvons aligner les groupes de ruches dans l'ordre d'importance des moyennes pondérées (tableau XII). Nous obtenons alors le tableau XIX qui met parfaitement en relief le 


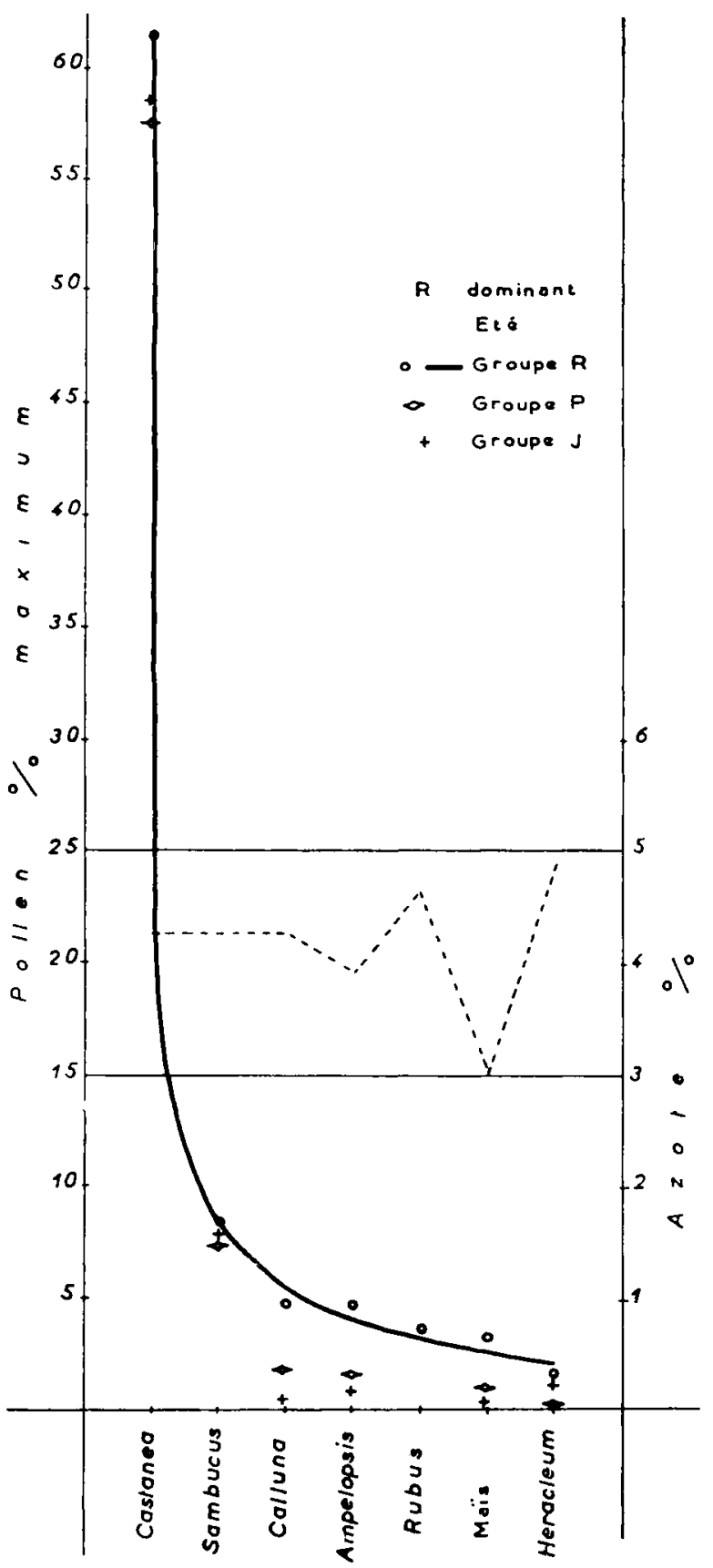

FIG. 38 (stite).

fait qu'au printemps la première et la deuxième colonnes sont occupées par les groupes $J$ et $P$ jusqu'à 4 p. Ioo d'azote, le groupe $R$ restant en $3^{\mathrm{e}}$ position ; de 3 , I p. IOO à $2 \mathrm{p}$. Ioo c'est presque toujours le groupe $\mathrm{R}$ 
qui occupe la première place. Enn été la situation est inverse, le groupe R étant fréquemment au premier rang (9 fois sur I3), les groupes $\mathrm{J}$ et $\mathrm{P}$ ayant perdu la première place même pour les pollens riches en azote.

\section{TABLEAT XIX}

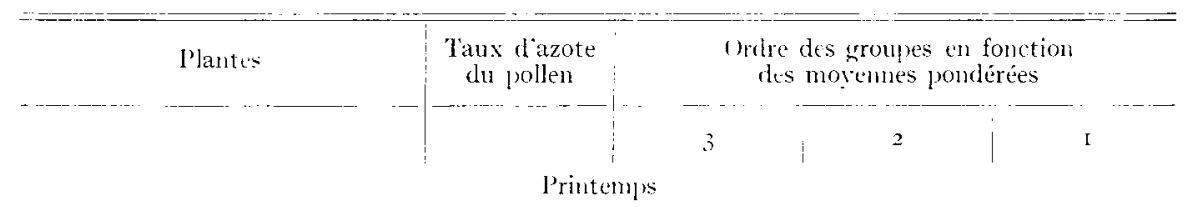

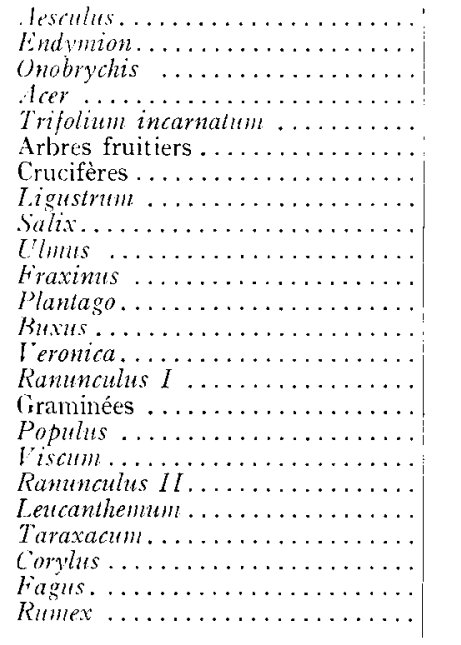

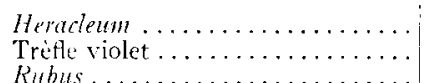

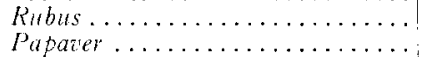

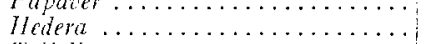

Tritolium repens

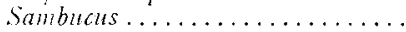

C'alluna...................

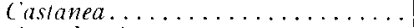

Ampelopsis...

Composées

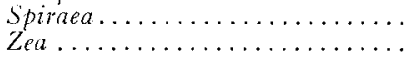

5,75
5,39
5,35
5,04
$5,0,3$
4,97
4,60
4,15
4,01
3,02
3,72
3,67
3,25
3,12
3,11
3,09
3,09
2,02
2,91
2,81
2,81
2,66
2,53
2,30
2,09

4,90

4,79

4,63

4,60

4,50

4,4 I

4,28

$4,2,3$

4,27

3,92

3,37

3,01

3,01

Été

Il ressort de cet ensemble de constatations que la teneur en azote des pollens semble jouer un rôle essentiel dans le choix des butineuses et que les différences qualitatives peuvent s'expliquer par une recherche d'une certaine qualité de nourriture, cette qualité étant assez étroitement liée à la teneur en azote du produit récolté. Il est bien évident qu'on ne peut supposer chez l'abeille une connaissance du taux d'azote des pollens qu'elle récolte; il est donc beaucoup plus vraisemblable que l'abeille soit capable d'apprécier par une voie sensorielle la "qualité alimentaire" du produit qu'elle recherche.

Un problème reste à élucider. Pourquoi ne retrouve-t-on pas unifor- 
mément chez toutes les colonies une préférence identique pour tous les pollens? Quel est donc le mécanisme qui conduit certaines colonies à rechercher des pollens spécialement riches en protéines alors que d'autres se contentent d'un régime moins substantiel ?

Il nous semble qu'il faille rechercher la réponse à cette question dans l'examen du cycle biologique de la colonie et que l'examen des résultats quantitatifs puisse nous aider à résoudre le problème.

$\mathrm{Si}$ nous nous reportons aux courbes de récolte des ruches caractéristiques (fig. 9) nous constaterons que dans la période printanière pen-

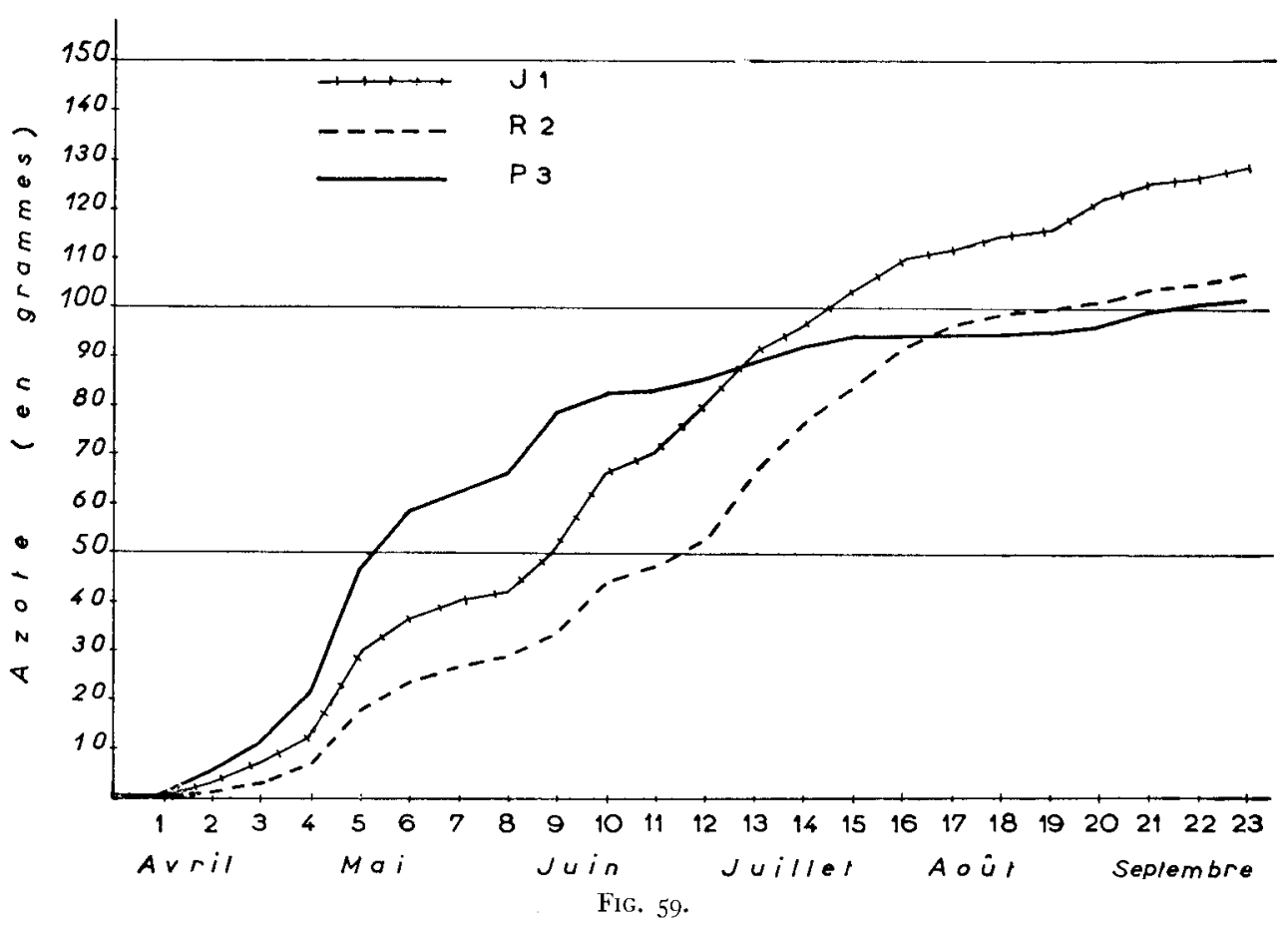

dant laquelle nous savons que les groupes $P$ et $J$ recherchent les pollens les plus riches en azote ces deux mêmes groupes sont ceux qui présentent les récoltes pondéralement les plus fortes. Ce sont aussi les ruches les plus développées. Par contre les ruches dı groupe $\mathrm{R}$ ne recherchent qu'un pollen plus pauvre en azote et en récoltent moins ; leur développement est par ailleurs moins avancé. Dans la période estivale on notera une baisse du taux d'azote dans les récoltes des groupes $\mathrm{P}$ et $\mathrm{J}$ et une diminution des apports de pollen ainsi qu'une baisse de développement de la colonie. De leur côté, les ruches du groupe $\mathrm{R}$ continuent à récolter des quantités appréciables de pollen plus riche en azote et leur développement ne manifeste pas de baisse importante. On examinera utilement la figure 59 qui représente la courbe cumulative des apports d'azote à la 
ruche (produit de la quantité de pollen par le taux d'azote) pour 3 colonies caractéristiques de chaque groupe. Cette figure montre très nettement le phénomène que nous venons de décrire.

\section{Conclusions des chapitres IV et V}

Nous sommes ainsi amenés à concevoir que l'activité de récolte d'une colonie est fonction de ses qualités potentielles et de son état physiologique. Une colonie en bon état physiologique et dotée des qualités requises récolte beaucoup d'un pollen à haute teneur en éléments nutritifs tandis qu'une colonie moins douée ne récolte qu'un pollen de moindre valeur et en quantités plus faibles. Ceci nous montre déjà qu'il n'y a pas régulation mais au contraire aggravation systématique d'un déficit de nourriture et développement exponentiel des colonies les mieux douées.

L'état momentané d'une colonie est la résultante d'un grand nombre de facteurs externes et internes; on peut d'abord distinguer des facteurs qui sont liés à l'histoire de la colonie, aux vicissitudes antérieures qui ont pu la marquer en bien ou en mal et qui n'ont qu'un caractère accidentel. Ensuite, des facteurs qui dépendent du patrimoine héréditaire et qui conditionnent ses aptitudes les plus diverses. Une colonie peut être dans un mauvais état physiologique par suite de conditions météorologiques défavorables par exemple mais posséder à l'état potentiel les qualités nécessaires pour surmonter rapidement 1'adversité. Réciproquement, une colonie disposant d'excellentes réserves à la suite d'une saison favorable dont elle n'a pas pu ne pas profiter restera une non-valeur d'un point de vue apicole si ses caractères génétiques d'aptitude au travail sont médiocres.

Nous trouverons 1'illustration de cette idée dans quelques exemples faciles à trouver au cours de notre travail. La colonie $\mathrm{J} \mathrm{2}$, accidentellement orpheline, donc en mauvais état physiologique, a fait preuve de qualités remarquables malgré son état. Qualitativement, ses récoltes sont peu différentes de celles des autres ruches du groupe J. Quantitativement, elles sont déficientes par suite du faible nombre des butineuses; dès que nous avons remédié à l'orphelinage par l'introduction de cadres de couvain nous avons provoqué un regain d'activité considérable qui dénotait une faculté élevée de rétablir la situation. Ia ruche 108 en I 953 s'est trouvée handicapée au départ par un hivernage défectueux mais ce handicap a été surmonté rapidement. I a ruche $J 5$ privée d'une partie de son couvain pour permettre le remèrage de la ruche $\mathrm{J} 2$ a récupéré très rapidement la substance perdue et a réussi à se trouver en excellent état en fin de saison. Toutes ces ruches, mises en mauvais état physiologique pour des raisons diverses ont réagi dans un sens favorable grâce à de bonnes qualités d'aptitude au travail. 
On peut envisager qu'une colonie possède un cycle annuel de développement qui est conditionné par l'adaptation au milieu extérieur dans lequel elle vit habituellement. Ce nilieu lui offre en quantités variables des aliments de qualité variable, le tout selon un cycle qui dépend de la flore et du climat du lieu. I'adaptation parfaite du cycle animal au cycle végétal aboutit à un équilibre qui assure à la colonie sa pérennité. Si l'adaptation est défectueuse les deux cycles ne coïncident plus parfaitement et la colonie se trouve en difficulté.

I a colonie travaillerait donc au cours de l'année selon un programme " préétabli en fonction du cycle annuel des floraisons du climat et du rythme de ponte de la reine. Des accidents peuvent se produire (accidents d'ordre météorologique ou d'ordre physiologique) qui amènent des décalages de ces cycles qui se traduisent par des retards ou des avances dont les conséquences peuvent être très importantes.

Dans les ruches du groupe $\mathrm{P}$ il y a indéniablement persistance d'un rythme de développement antérieurement acquis dans une région précoce. Ia période printanière est marquée par des apports massifs d'un pollen riche en azote favorisant à l'extrême le développement du couvain. La reine pond selon le rythme acquis. Les colonies s'assurent une avance importante sur les autres pendant quelques semaines. Par contre, en été, on assiste à une véritable estivation avec diminution de la ponte, réduction des apports de pollen et polarisation sur des plantes fournissant un pollen moins riche en azote.

Les ruches du groupe J sont un peu moins précoces et paraissent adaptées à un rythme des floraisons plus voisin de celui de la région parisienne. On ne trouve pas en été la même tendance à l'estivation que dans. le groupe $P$.

Quant aux ruches du groupe $\mathrm{R}$, nous pensons qu'il s'agit de colonies présentant un rythme très tardif, en tout cas mal adapté aux floraisons du bassin parisien bien que leur origine géographique soit relativement. proche.

Récolte du pollen par un essaim naturel. - Nous trouverons dans le comportement de l'essain une confirmation de notre hypothèse d'une adaptation à un rythme biologique prédéterminé. Il s'agit, avec l'essaim. naturel, d'un cas tout à fait particulier. Nous avons comparé le comportement de la souche et de l'essaim naturel qui en provient, les deux colonies restant à proximité l'une de l'autre. Nous avions tout d'abord pensé trouver dans les deux colonies sours un comportement rigoureusement semblable, l'essaim continuant à risiter les mêmes plantes que la souche. Le 5 juin I953, une de nos colonies ayant essaimé, nous avons posé une trappe à pollen sous la souche et sous l'essaim mis en place à quelque distance de la souche. Nous avons alors constaté un total changement dans la flore visitée par l'essaim par rapport à la souche. La quantité de pollen 
récoltée d'abord très faible chez l'essaim a cru rapidement et a, vers le ro juin dépassé celle de la souche. Le taux d'azote dans les récoltes s'est révélé très différent, l'essaim ayant une tendance marquée à récolter des pollens plus riches en azote que la souche (tableau XX). Or, on sait que l'essaim naturel est caractérisé par des besoins très importants puisqu'il ne possède aucune réserve et aussi par un dynamisme considérable qui 1'amène à réaliser des performances très supérieures à celles d'une ruche établie. I1 représente donc un organisme neuf qui rompt totalement avec la souche dont il provient et qui adopte immédiatement un rythme nouveau adapté à des besoins différents. La plus grande aptitude à récolter du pollen et le choix des pollens à haute valeur nutritive sont le signe d'une adaptation à une situation nouvelle et du commencement d'un nouveau cycle décalé par rapport au précédent. Il y a là une sorte de rajeunissement.

\section{TABLEAU XX}

\begin{tabular}{|c|c|c|c|c|}
\hline \multirow{2}{*}{ Dates } & \multicolumn{2}{|c|}{ Souche } & \multicolumn{2}{|c|}{ Essaim } \\
\hline & Poids de la récolte & $N \%$ & Poids de la récolte & $N \%$ \\
\hline juin 1953 & $g$ & & $g$ & \\
\hline $5 \ldots \ldots \ldots \ldots \ldots$ & 10,85 & 3,36 & 0 & \\
\hline $6 \ldots \ldots \ldots \ldots \ldots$ & $\mathrm{I}_{3,20}$ & 357 & o & \\
\hline $7 \ldots \ldots \ldots \ldots \ldots$ & 5,40 & - & 0,08 & \\
\hline$s \ldots \ldots \ldots \ldots$ & $\mathrm{T}, 75$ & - & 0,30 & \\
\hline $9 \ldots \ldots \ldots \ldots$ & 3,47 & - & 0,35 & \\
\hline Io........ & 17,60 & 3,57 & 2,80 & 3,50 \\
\hline I $1 \ldots \ldots \ldots \ldots$ & 16,10 & $2,4+4$ & 3,35 & \\
\hline $12 \ldots$ & 10,55 & $2,+4$ & 8,35 &,+ 06 \\
\hline $14 \ldots \ldots \ldots \ldots \ldots$ & 29,25 & 3,08 & 28,35 &,+ 06 \\
\hline & 7,40 & $.3,85$ & $25, \mathrm{I} 5$ & 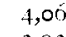 \\
\hline $10 \ldots \ldots \ldots \ldots$ & 9,35 & 3,85 & 20,00 & 3,93 \\
\hline${ }_{1}{ }_{7} \ldots \ldots \ldots \ldots$ & 7,20 & 3,85 & 26,50 & 4,20 \\
\hline $18 \ldots \ldots \ldots \ldots \ldots$ & 7,85 & 3,99 & 16,70 & 4,00 \\
\hline $19 \ldots \ldots \ldots \ldots \ldots$ & 19,40 & 3,64 & 26,82 & 4,00 \\
\hline
\end{tabular}

Ce retour à un état primitif que constitue l'essaimage naturel est un phénomène brutal. Brusquement, un groupe d'abeilles se trouve devant des nécessités nouvelles, des bescins différents de ceux de la veille. L'adaptation est rapide et même pratiquement instantanée. Les butineuses qui ont perdu le sens de l'orientation de la souche présentent une grande plasticité pour se polariser sur de nouvelles sources de nourriture mieux adaptées à leurs besoins nouveaux. Il n'en est pas de même si artificiellement nous créons une situation nouvelle; la réponse n'est pas immédiate. Nous avons opéré le 3 aôit I953 une permutation de deux ruches situées à quelques mètres l'une de l'autre et munies chacune d'une trappe à pollen. Ces deux ruches présentaient depuis plusieurs jours des récoltes profondément différentes ; un simple coup d'œil sur le contenu des trappes montrait dans l'une des colonies un pollen gris (Vicia) presque pur alors que dans l'autre ce pollen était absent et remplacé par un mélange de 
Crucifères et de Composées. A près la permutation opérée le matin de bonne heure, nous avons constaté que cette opération qui n'avait pas affecté les butineuses puisque celles-ci reviennent toujours au même emplacement, avait laissée intacte la composition des récoltes. I a ruche $\mathrm{A}$, possèdant les butineuses de $\mathrm{B}$ présentait les mêmes récoltes que $\mathrm{B}$ la veille de la permutation et réciproquement. Il n'y a donc pas eu une adaptation immédiate à une situation nouvelle; cette expérience ne pouvait malheureusement pas être poursuivie puisque nous ne pouvions pas savoir a priori comment auraient évolué dans les jours suivants les récoltes de chaque ruche. Mais il est intéressant de noter que notre opération de permutation réalisant une situation totalement artificielle n'a provoqué aucune réaction immédiate dans la polarisation des butineuses.

Ceci nous confirme encore dans notre opinion selon laquelle il existe pour chaque colonie un rythme interne dépendant peut-être de la reine et qui constitue un véritable programme qui ne peut se modifier que sous l'action de facteurs externes ou internes puissants.

Si nous comparons entre elles nos expériences de I950 à I955 nous constatons plusieurs faits importants :

- Les expériences portant sur un petit nombre de ruches (5 en I950, $53,54)$ sont insuffisantes pour mettre en lumière les phénomènes que nous avons découverts en I955 en travaillant sur des ruches plus nombreuses et constituant des lots distincts. Certains faits tels le rapport entre la valeur nutritive des pollens et le choix qui en est fait par les abeilles ne peuvent apparaitre clairement que sur un nombre suffisant de colonies. Aucune règle n'est absolue et nous dégageons surtout des tendances générales qui n'apparaissent pas sur un tout petit lot de ruches.

- Nous noterons encore un autre fait qui pent avoir une grande signification : c'est l'existence en 195:5 d'une densité de ruches exceptionnelle sur le territoire de la Station. Nous avons dénombré en juin I955 plus de Ioo ruches dans un périmètre de quelques centaines de mètres alors que dans les autres années nous n'avions guère que 30 à 40 ruches au même endroit pendant la saison apicole. Il s'est donc produit en I955 une concurrence beaucoup plus intense entre les colonies, ce qui peut avoir contribué à accentuer les différences constatées entre les ruches et, en même temps, à étendre la gamme des plantes visitées par les abeilles, gamme relativement moins étendue dans les années précédentes. Ainsi, l'utilisation massive par les abeilles des pollens de Rumex, Ranunculus, Graminées, etc. pauvres en azote s'expliquerait comme la conséquence d'une concurrence importante entre ruches et d'un manque de pollen de bonne qualité à certains moments. I a faiblesse des récoltes de miel dans une année considérée comme bonne s'expliquerait de même par le nombre trop élevé des ruches dans le secteur expérimental. Nous devrions donc, 
dans une certaine mesure, au hasard d'une surpopulation, d'avoir vu prendre un caractère particulièrement net à un phénomène qui peut, en bonne année et avec une faible densité de ruches, passer à peu près inaperçu.

\section{Chapitre: VI}

\section{RECHERCHES SUR LES SUBSTANCES ATTRACTIVES I) U POLLEN}

Nous avons vu précédemment que l'abeille paraît orienter son choix vers tel ou tel pollen en fonction de données précises et complexes qui font intervenir l'abondance de la plante qui le fournit dans le milieu environnant, la présence ou l'absence de nectar et certains caractères liés très probablement à la valeur nutritive du pollen dont le critère semble être la teneur en azote. Nous ignorons encore le mécanisme de la perception de la valeur nutritive d'un pollen. Il est possible que ce mécanisme fasse intervenir le goût et l'odorat puisque les pièces buccales, riches en terminaisons sensorielles, interviennent dans la confection des pelotes. Certaines observations peuvent nous permettre de confirmer cette hypothèse. ItINDAUEr (I948) a montré le rôle de l'odeur dans la récolte du pollen mais ses observations restent fragnentaires. Il a pu inhiber les danses des récolteuses de pollen de Corylus en y ajoutant du poivre en concentrations croissantes; par ailleurs, il a réussi à faire récolter de la fécule en lui communiquant l'odeur des feuilles d'Absinthe.

Mais les observations essentielles sont les suivantes:

a) En cas de disette, l'abeille peut récolter ì peu près n'importe quel corps pulvérulent non répulsif. Les observations à ce sujet sont légion. On a vu les abeilles récolter de la sciure de bois, du poussier, des fibres de pâte à papier, des farines et même la poussière des chemins. RFNnER (communication orale) a même observé dans ses chambres d'élevage des abeilles totalement dépourvues de pollen et qui exécutaient à vide, sur le sol, les mouvements typiques de récolte.

b) I orsqu'on offre aux abeilles des produits de remplacement du pollen, levures, farines diverses ou mélanges complexes on peut facilement obtenir la confection des pelotes pourru que le pollen naturel fasse plus ou moins défaut. Dès que les sources normales de pollen apparaissent, le butinage sur les surrogats cesse totalement, quelles que soient la qualité et la valeur nutritive des produits, valeur que l'on peut mettre en évidence au laboratoire par des tests physiologiques sur les glandes pharyngiennes, les ovaires, le corps adipeux, la longévité.

c) Lorsque l'abeille peut, dans la nature, exercer son choix dans la récolte du pollen, elle utilise de préférence certains pollens à d'autres. 
Le pollen des Conifères, par exemple, pourtant fort abondant et facile à récolter ne l'est qu'en cas de pénurie et dans des proportions relativement très faibles. On sait par ailleurs que le pollen des Conifères est biologiquement inactif.

d) Lorsque l'abeille visite certaines plantes telles que les Cucurbitacées (Percival, I955) ou Helianthus annuns, il arrive fréquemment que la collecte du nectar la couvre de pollen. Au lieu de récolter ce pollen abondant et d'en faire des pelotes on la voit s'en débarrasser soignentsement comme si ce pollen la gênait. Ce comportement est inexplicable si l'on n'admet pas que l'abeille opère un choix.

e) La corrélation entre la quantité de pollen offerte par fleur et par jour et la récolte ou non récolte par les abeilles est à peu près nulle ainsi que l'a montré PERCIVAL (1955). Ce n'est done pas la facilité de récolte qui guide la butineuse contrairement à ce qui se passe pour le nectar, tout au moins en partie.

f) I,orsque, dans des conditions artificielles, on donne le choix aux abeilles entre différents pollens, elles opèrent une sélection en se polarisant sur certains d'entre eux. C'est ce qu'ont montré SiyNGE (I947) ainsi que I,EVIN et BOHAR'T (I95.5) qui n'ont d'ailleurs tronvé aucune corrélation entre le choix et la teneur en azote des pollens.

Toutes ces observations nous amènent à considérer que le pollen constitue pour l'abeille un produit parfaitement défini, possédant des qualités qui forment une cascade de stimuli sensoriels hiérarchisés. On peut déjà affirmer que la qualité de pulvérulence est la qualité de base. I,orsque toutes les autres qualités ont disparu, tout corps pulvérulent est assimilé à du pollen si le besoin en pollen est impérieux et l'offre nulle : c'est le cas du butinage des poudres inertes. Apparaissent ensuite deux qualités qui sont, par ordre d'importance :

I $^{\mathrm{O}}$ Le gônt ou l'odeur, ou bien le goût et l'odeur du pollen ;

$2^{\circ}$ La valeur biologique et nutritive du produit.

Un produit nutritif riche en azote et biologiquement actif est délaissé au profit du plus mauvais pollen, mais qui "sent " le pollen. Un bon pollen est préféré à un mauvais pollen pourvu que sa qualité de pollen soit reconnue par l'abeille.

I'abeille cherche donc un corps pulvérulent, à odeur ou saveur de pollen, aussi nutritif ou biologiquement actif que possible. Cette hypothèse permet d'expliquer pourquoi certains pollens riches en azote (Medicago sativa) sont négligés et pourquoi des pollens de valeur nutritive médiocre (Composées en général) sont malgré tout régulièrement récoltés. Les premiers sont considérés par l'abeille à l'égal des meilleurs succédanés qui sont pel1 attractifs parce que dépourrus de la substance qui caractérise la matière pollen, les seconds sont attractifs parce que riches en cette substance mais ils seront toujours détrôniés au profit 
du pollen idéal qui comporte à la fois la substance attractive et une haute valeur nutritive, et dont le prototype nous semble être le pollen d'Onbrychis. Rien ne prouve d'ailleurs que la substance attractive soit dépourvue d'un rôle biologique dans la ruche.

'our vérifier cette hypothèse générale il nous fallait isoler la substance attractive du pollen. C'est ce que nous avons fait en utilisant les niiéthodes que nous allons maintenant décrire.

\section{A. -- MÉTHODES}

Nos expériences sur la substance attractive du pollen se sont déroulées à l'extérieur en dressant des butineuses à venir récolter du pollen sur une tablette dressée en plein air. Notre dispositif comporte une plaque de verre de to $\times 30 \mathrm{~cm}$ sur laquelle on colle arec du miel des anneaux de verre de $2 \mathrm{~cm}$ de diamètre et de $\mathrm{I} \mathrm{cm}$ de hauteur. Ces anneanx peuvent etre remplis de pollen ou de poudres à tester. I'anırçage du dispositif a lieu par capture de butineuses au trou de vol d'une ruche ; ces butineuses ne sont relâchées qu'après s'être gorgées de miel à l'endroit de la tablette. Lorsque l'amorçage est réalisé on substitue à la coupelle de miel le dispositif à anneaux de verre. Un scul amorçage suffit en général pour plusieurs semaines car les abeilles dressées reviennent régulièrement si les expériences ont lieu chaque jour.

Ne retrouvant plus la coupelle de miel, les butineuses sucent le miel à la base des anneaux et découvrent rapidement qu'ils contiennent du pollen. I,eur comportenent est alors caractéristique : posées sur le bord de l'anneau elles plongent leurs pièces buccales dans le pollen ainsi que la première paire de pattes et, d'un nouvement très vif, se couvrent la partie antérieure du corps de pollen ; ensuite elles s'envolent et exécutent les mouvements classiques décrits par CasTFEL (IgI2), formant des pelotes parfaitement régulières. Dès que le niveau du pollen baisse dans l'annean elles y pénètrent en entier et se frottent par toute la partie inférieure du corps contre la surface du pollen. Le travail est parfois interrompu pour un léchage des traces de niel qui subsistent à la base des anneaux.

I orsque le pollen est du pollen entier, non fractionné, récolté à la main (Zea, Pinus, Corylus, Alnus) ou provenant du broyage des pelotes récoltées à la trappe, les mourements de récolte sont très vifs, ne comportant ancune hésitation; î semble qu'à distance, sans doute grâce à l'odorat, le produit soit parfaitement reconnu comme du pollen. I,orsqu'il s'agit d'une poudre inerte on d'un pollen épuisé l'abeille tourne en volant autour de l'anneau, s'en approche mais se pose rarement. Si elle se pose, elle plonge inmédiatement son proboscis dans la poudre et s'envole aussitôt en se débarrassant du produit comme si elle venait de commettre 
une erreur. Si l'on tasse soigneusement la surface de la poudre à l'intérieur de l'anneau on retrouve au bout d'un certain temps les marques laissées par les pattes et les mandibules des quelques butineuses qui ont fait des essais infructueux de récolte. I a surface de verre est suffisamment grande pour y coller de I6 à 25 anneaux que l'on dispose, comme il se doit, en carré latin, chaque produit comportant 4 on 5 répétitions. On observe bien entendu la consommation du pollen qui est le critère de l'attractivité. Cette consommation est rapide lorsque le produit est très attractif. Deux témoins sont toujours ménagés : 1'un est un pollen entier totalement attactif, l'autıe, un pollen épuisé, totalement uu presque totalement inacpif. I a consommation commençant toujours par le pollen entier on n'observe en général le butinage sur les éléments noins attractifs provenant de fractionnements qu'avec un certain retard et lorsque le pollen entier commence à s épuiser.

On pourrait, à la rigueur, tester par cette méthode la valeur attractive des différents pollens naturels mais nous verrons plus loin qu'une autre méthode s'est révélée plus intéressante pour cet objet. En effet, on constate pendant les périodes oì le dressage est possible, c'est-à-dire par temps de disette relative, que tous les pollens naturels exercent une attraction suffisante pour que la distinction devienne trop difficile. Si la disette relative cesse, ce sont les fleurs qui deviennent beaucoup plus attractives que notre platean et celui-ci est rapidement déserté. Par contre, la méthode est parfaite pour tester la valeur attractive des divers produits de fractionnement obtenus à partir du pollen entier. La principale difficulté est d'obtenir tout d'abord un pollen rigoureusement inactif ou tout au moins d'une activité suffisamment faible pour que le butinage soit pratiquement inhibé. Une fois ce pollen obtenu il est facile de lui restituer en proportion convenable les différentes fractions qu'on lui a enlevées et ainsi, de proche en proche, de connaitre, à un état de pureté de plus en plus grand, les fractions attractives du pollen c'est-à-dire celles qui restituées à du pollen inactif lui rendent son attractivité.

\section{B. - RESULTATS DES ESSAIS DE FRACTIONNEMENT DU POLLEN}

Ainsi que nous l'arons signalé plus haut, la première difficulté était d'obtenir un pollen totalement inactif. Nous y sommes parvenus par extraction par l'alcool éthỵlique au soxhlet pendant huit jours. I. e résidu brun obtenu, remis en poudre fine est totalenent inactif nuais il est butiné normalement si on lui restitue des portions actives de l'extrait. Tin opérant l'extraction au Soxhlet pendant huit jours on obtient donc un pollen inactif mais l'extrait l'est aussi car le chauffage prolongé détruit les substances intéressantes. I1 faut donc séparer les deux opérations ; on pré- 
pare d'une part le pollen inactif servant aux tests, et on ne peut l'obtenir que par chauffage prolongé; d'autre part il faut procéder à des extractions plus ménagées pour obtenir les fractions actives sans les altérer.

Nous résumerons les opérations de fractionnement effectuées, la méthode de test employée étant toujours la mêne. I a réponse obtenue des abeilles étant toujours claire et sans équivoque, la fraction testée est, soit d'inactivité totale, et comparable à celle du témoin inactif, soit active et les abeilles la récoltent pour en faire des pelotes. Nous arons constaté que plus le fractionnement est poussé plus il est difficile d'obtenir un butinage actif en restituant le produit à la dose supposée normale. Cela tient certainement à des pertes importantes de substance en cours de fractionnement, surtout par chromatographie. Sur la fraction active $n^{\circ}$ I6 par exemple nous avons pu montrer que l'attractivité est proportionnelle à la concentration.

On peut dire à la suite des essais de fractionnenent que la partie attractive du pollen est une substance passant dans l'éther sulfurique à troid, distillant sous $0,04 \mathrm{~mm}$ à $190^{\circ}-2 \mathrm{IO}^{\circ}$, insaponifiable, présentant un test de Lieberman positif et qui purifiée par chromatographie sur alumine se présente comme une substance ou un groupe de substances susceptibles d'être voisines des stérols.

On savait déjà que le pollen est riche en stérols, notamment en phytostérols; le résultat obtenu n'est done pas tellement surprenant. I1 s'agit certainement de substances spécifiques car tous les essais que nous avons pu faire avec différents stérols purs et de substances roisines. (Acide cholique, Coprostanone, Vitanine Ii, Coprostérol, Zymostérol, 1)ihydrocholestéro1, Eupicoprostérol, Iqquiline) sont restés totalenent négatifs. I,es recherches se poursuivent actuellenent pour acherer de déterminer plus précisément la formule chinic|ue du ou des corps anxiuels le pollen doit son attractivité pour l'abeille.

\section{C. - AUtRES ESSAIS}

Dans une seconde série d'essais nous avons opéré en nilien clos. Tne ruchette normale de 3 calres étant placée clans une petite serre de $10 \times 3 \times 2,5$ m, les abeilles sont labituées à venir chercher da sirop de sucre dans des nourrisseurs. Après quelques jours de perturbation causée par l'installation en milieu clos, les butinenses finissent par s'habituer à visiter les coupelles et le butinage devient actif. On peut alors disposer des coupelles pleines de pollen et hâter leur déconverte en les juxtaposant à des nourrissenrs. Is butinage commence avec quelque difficulté puis il devient très régulier et plusienrs centaines de grammes de pollen sont ainsi absorbés chaque jont. I a rucle présente nu convain 
normal et peut même dans une certaine mesure se développer. Ces résultats confirment ceux de Mac GREGOR (1952).

Placée fin mai I956 en serre avec un cadre de couvain unique notre ruchette avait trois cadres au bout de deux mois de ce régime et une population normale. Iaute de pouvoir chauffer la serre nous avons dû interrompre nos essais en octobre mais pendant tout l'été nous avons disposé d'une population très suffisante pour nos essais qui portèrent surtout sur le choix de différents pollens par les abeilles.

Nous avons testé différents mélanges de pollen présentant toutefois une dominante florale très nette; il s'agissait de pollens récoltés à la trappe et remis en poudre très fine soigneusement tamisée. Si l'on place aux quatre coins de la serre quatre coupelles identiques contenant une quantité égale d'un pollen uniforme on obtient un butinage égal sur les quatıe coupelles. I1 n'y a done pas de site préférentiel. Lorsqu'on remplace le mélange habituel par des pollens à forte dominance d'une espèce (Ciste, Eucalyptus, Bruyère arborescente, Sainfoin) on constate que la répartition des butineuses cesse rapidement d'être uniforme, une coupelle étant rapidement 1'objet d'un butinage intense et les autres pratiquement délaissées. Dans notre expérience, les pollens de Ciste, de Bruyère et d'Eucalyptus sont délaissés au profit du pollen de Sainfoin qui fait preuve d'une attractivité considérable et qui capte toutes les butineuses à son profit. Si l'on permute les coupelles il suffit de quelques minutes pour que les abeilles qui ront jusqu'à suivre la coupelle dans son déplacement retrouvent le nouvel emplacement. Il existe donc des pollens exerçant une forte attractivité et qui concurrencent très efficacement d'autres pollens moins attractifs. Nous remarquerons au passage que le pollen dc Sainfoin est plus riche en azote que les autres pollens testés au cours de 1'essai. Ia figure 60 montre la différence d'activité des butinetises sur pollen de Sainfoin et sur pollen de Ciste.

\section{CHAPI'TE VII}

\section{LA FLOIRE UTILSEE PAR I.ES ABEILLES}

Si les rapports entre l'abeille et les plantes qu'elle visite ont fait l'objet de nombreuses études de la part des spécialistes de la biologie florale et de la pollinisation, on peut dire que les relations qui existent entre la flore d'un lieu, prise dans son ensemble et la population des abeilles de ce même lieu sont beaucoup moins bien connues.

Ces deux aspects du problème des relations fleurs-insectes sont bien distincts. Dans le premier cas on ne considère que l'activité déployée par une abeille déterminée au niveau de l'individu végétal ; dans le second 


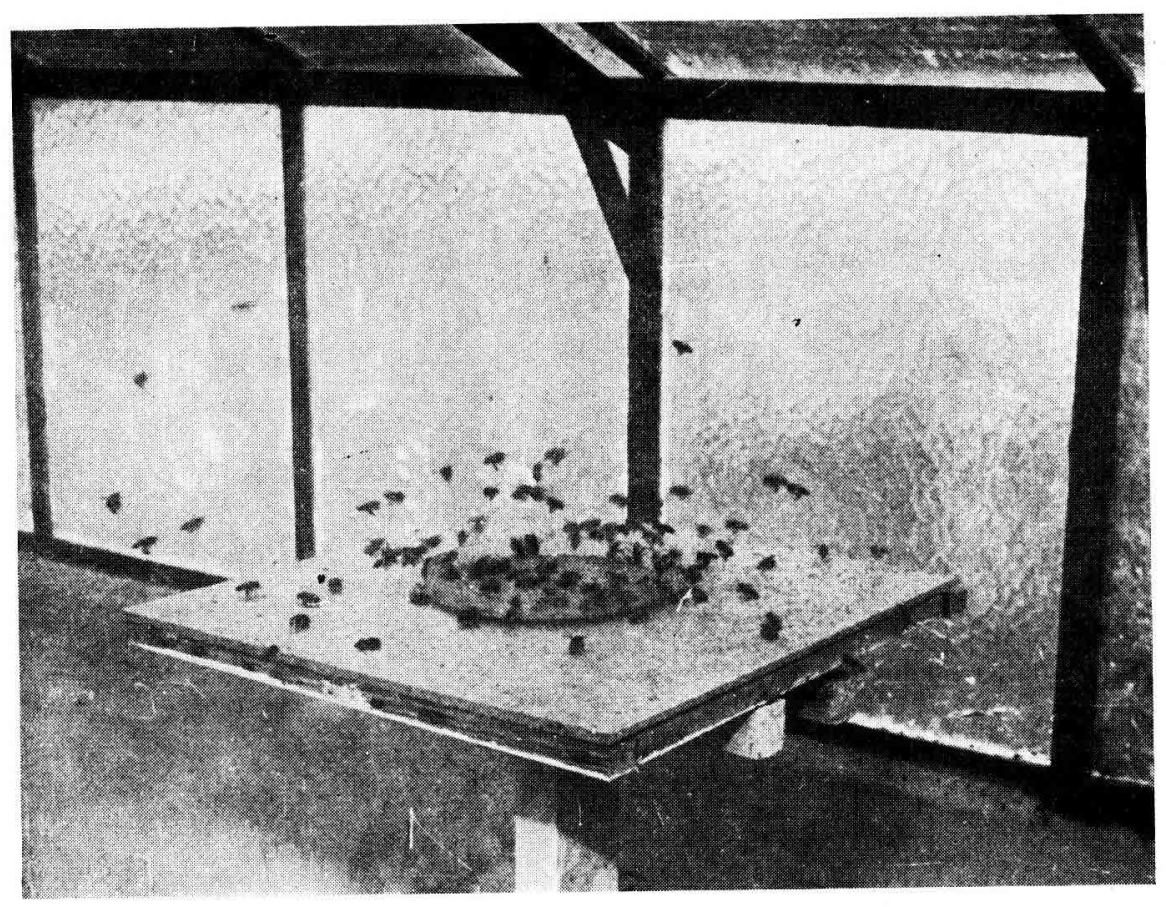

Sainfoin

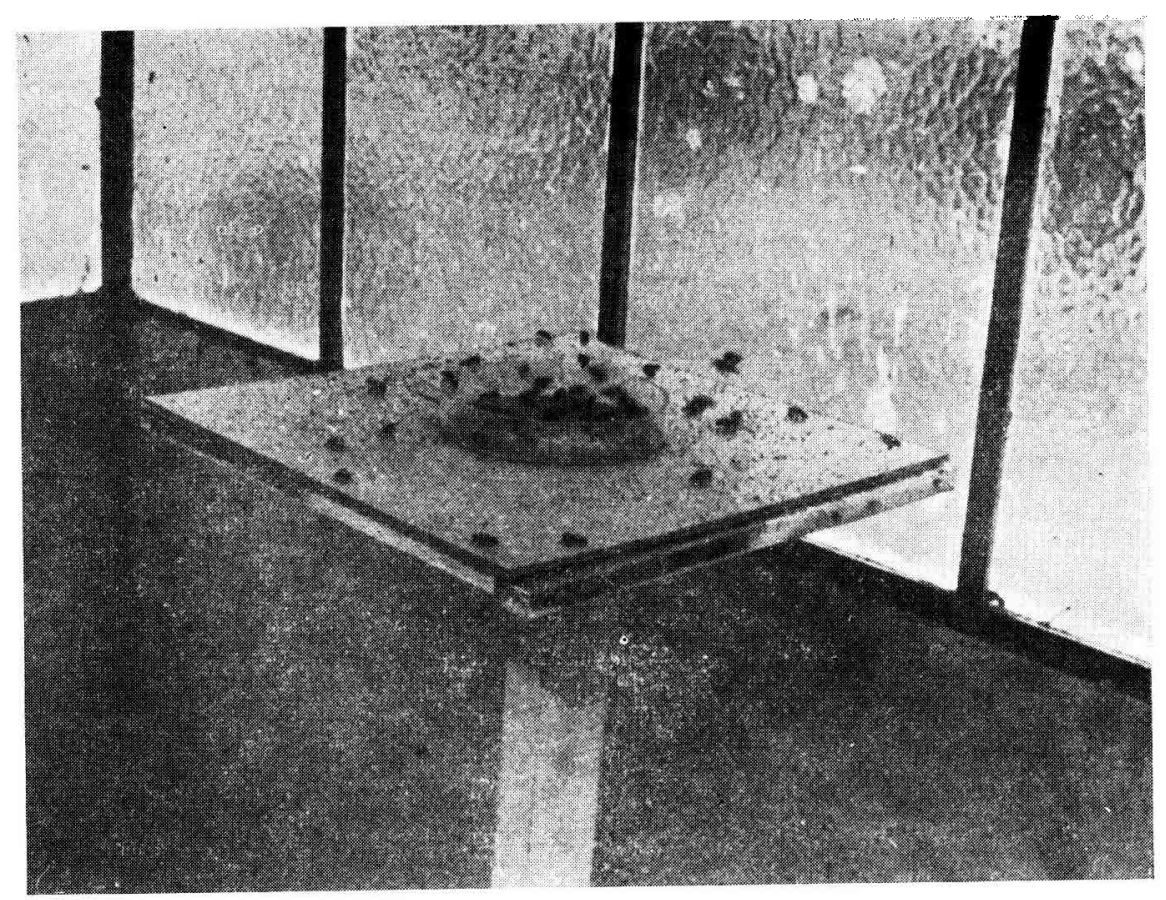

Ciste

Fig. 60. 
cas c'est toute la flore qui est en cause et ceci met en jeu tous les processus de choix de la part des abeilles et de concurrence entre plantes du côté des végétaux. Nous savons déjà qu'au sein de la flore disponible l'abeille opère un choix et n'utilise qu'une partie des plantes pour sa nourriture. Nous savons aussi que ce choix semble s'opérer dans un sens bien déterminé et que la valeur nutritive des produits récoltés est à sa base. II est donc intéressant de faire l'inventaire des plantes que l'abeille recherche et de voir dans quelle mesure il est influencé par la localité. Nous possédons une masse suffisante de documents provenant de plusieurs points de l'Europe pour pouvoir nous permettre quelques comparaisons utiles. D'autre part nous essayerons de comparer entre eux différents biotopes bien définis tels que la forêt, la garrigue, la lande, la prairie quant à leur valeur pour l'abeille et nous nous efforcerons d'en tirer diverses conclusions théoriques et pratiques.

\section{A. - LA FLORE UTILISÉE PAR L'ABEILLE EN DIVERS PAYS D'EUROPE DONT LA FRANCE}

I a prenière constatation que nous ferons, en accord avec les différents auteurs qui ont étudié la récolte du pollen par les abeilles, c'est que le nombre des espèces végétales utilisées est très faible si on le compare au nombre des espèces présentes en un lieu. I.es espèces et groupes d'espèces rraiment essentiels sont en nombre infime et ne dépassent pas quelques dizaines.

Percival (I947) ayant recensé autour de son rucher expérimental 225 espèces botaniques différentes n'en a retrouvé que 86 qui soient utilisées par les abeilles. Parmi ces plantes, dont 66 identifiées avec certitude, I7 seulement ont une importance réelle et fournissent la plus grosse masse du pollen récolté. Parmi ces I7 plantes, I2 sont très communes, 5 sont seulement communes. I,es plantes dont le pollen est récolté en abondance sont presque toutes des plantes livrant du nectar.

Malirizio (i953) arrive aux mêmes conclusions. Selon des observations faites pendant plusieurs années et en divers points de la Suisse, le nombre des espèces végétales ayant une signification pour la récolte du pollen par les abeilles n'atteint tout au plus que quelques dizaines. Par contre, le nombre des plantes secondaires est assez élevé mais au total ces plantes restent très peu importantes.

Srage (I947), Jaxthimer (I949), Schwan et Mar'inov (I954), Parker (I926), I ordre. Il est donc intéressant de rechercher dans quelle mesure leurs données se recoupent quant à l'importance des différentes plantes. Mais pour pouvoir faire utilement cette comparaison il est indispensable que nous 
- 5 Production de moins de $100 \mathrm{kgs.}$

066 - $\quad$ - plus de $100 \mathrm{kgs}$.

- Limites des régions de production.

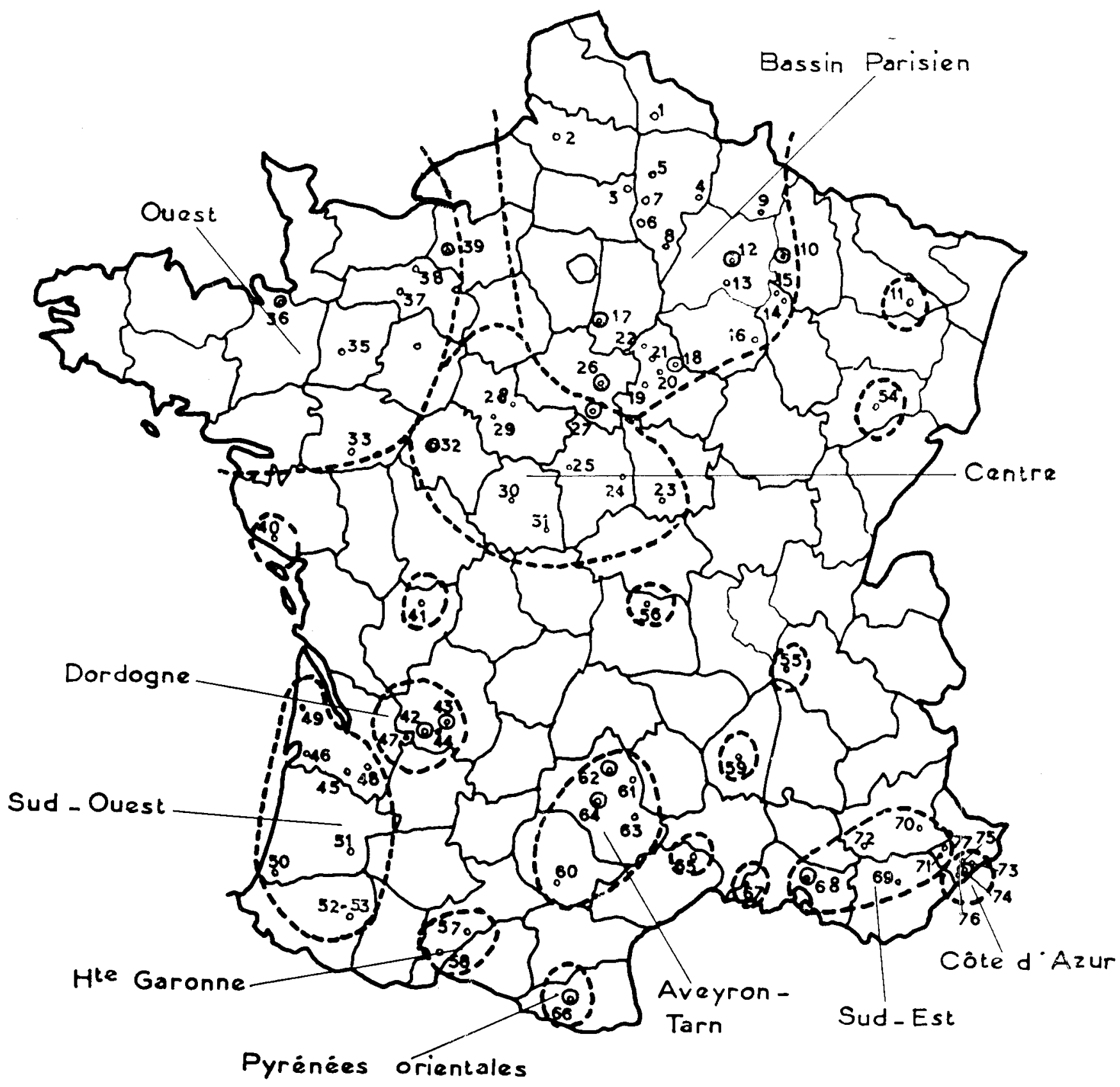

PRODUCTION DE POLLEN - ANNÉE APICOLE 1955 

regroupions nos propres données qui ont été acquises tant à Bures que sur l'ensemble du territoire français.

Nous disposons en ce qui concerne la flore ntilisée en lirance par l'abeille pour la récolte du pollen d'un nombre de données relativement iêmportant. Fin effet, nous connaissons les plantes utilisées à Bures-surYvette au cours des années 1950 (Cévrier à juin), I953 (mars-avril) r954, (mars avril), I955 (mars à fin septembre). I'tune année à l'autre nous avons retrouré sensiblement les mêmes plantes, tout au moins en ce qui concernc les floraisons essentielles; une plus grande variabilité a été notée dans les plantes secondaires.

Nous disposons en outre des résultats d'une vaste enquête portant sur l'ensemble du territoire français et sur quelques points de l'Afrique du Nord. Une société de produits diététiques nous ayant confié de I954 à I956 le contrôle botanique des lots de pollen qu'elle achetait en lirance aux apiculteurs pour la préparation d'un produit diététique, nous avons eu entre les mains un nombre important d'échantillons représentant des milliers de récoltes faites à la trappe à pollen sur une grande partie du territoire. Nous avons étudié ces échantillons par les méthodes décrites an chapitre II et sur lesquelles nous ne reviendrons pas.

Le tableau XXI résume les données relatives aux analyseseffectuées pendant les trois années de l'enquête.

\begin{tabular}{l} 
TABLEAU XXI \\
\hline
\end{tabular}

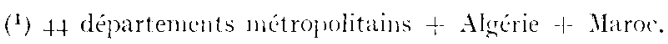

La lecture de ce tableau permet de constater que la récolte du pollen par les apiculteurs a tendance à se concentrer dans un petit nombre de régions et dans un petit nombre de mains. I a quantité produite par apiculteur récoltant indique que les plus gros producteurs ont tendance à augnenter chaque année leur production tandis que les petits producteurs ou les producteurs mal équipés renoncent à la récolte du pollen, ce qui prouve que toutes les régions 11'ont pas la nlême valeur pour la récolte et que la technique de récolte n'est pas encore parfaitement connue à l'heure actuelle des apiculteurs amateurs.

Pour confronter nos résultats et ceux des auteurs etrangers nous avons dressé 1e tableau XXII qui regroupe par familles botaniques les plantes citées par Matrizio pour la suisse, 'ERCIVAI, et SYNGE pour la 
Grande-Bretagne, Schwan et Martinov pour la suède, Jaxtheimer pour 1'Allemagne et par nous-mêmes pour la Iirance (Bures seul, France entière). Nous avons enfin ajouté en dernière colonne les indications données par PARkfr sur la récolte du pollen dans l'état d'Iowa (U. S. A.). Le travail de PARkER ne comportant pas d'estimation des importances relatives des différentes plantes nous avons seulenent porté des astérisques en regard des genres qu'il cite. Si la plupart des travaux tiennent compte de la flore pollinifère dans son ensemble, le travail de PERCIVAL se rapporte exclusivement à la période allant du 28 avril au 23 aồt I 945 .

\section{TABIEAU XXII}

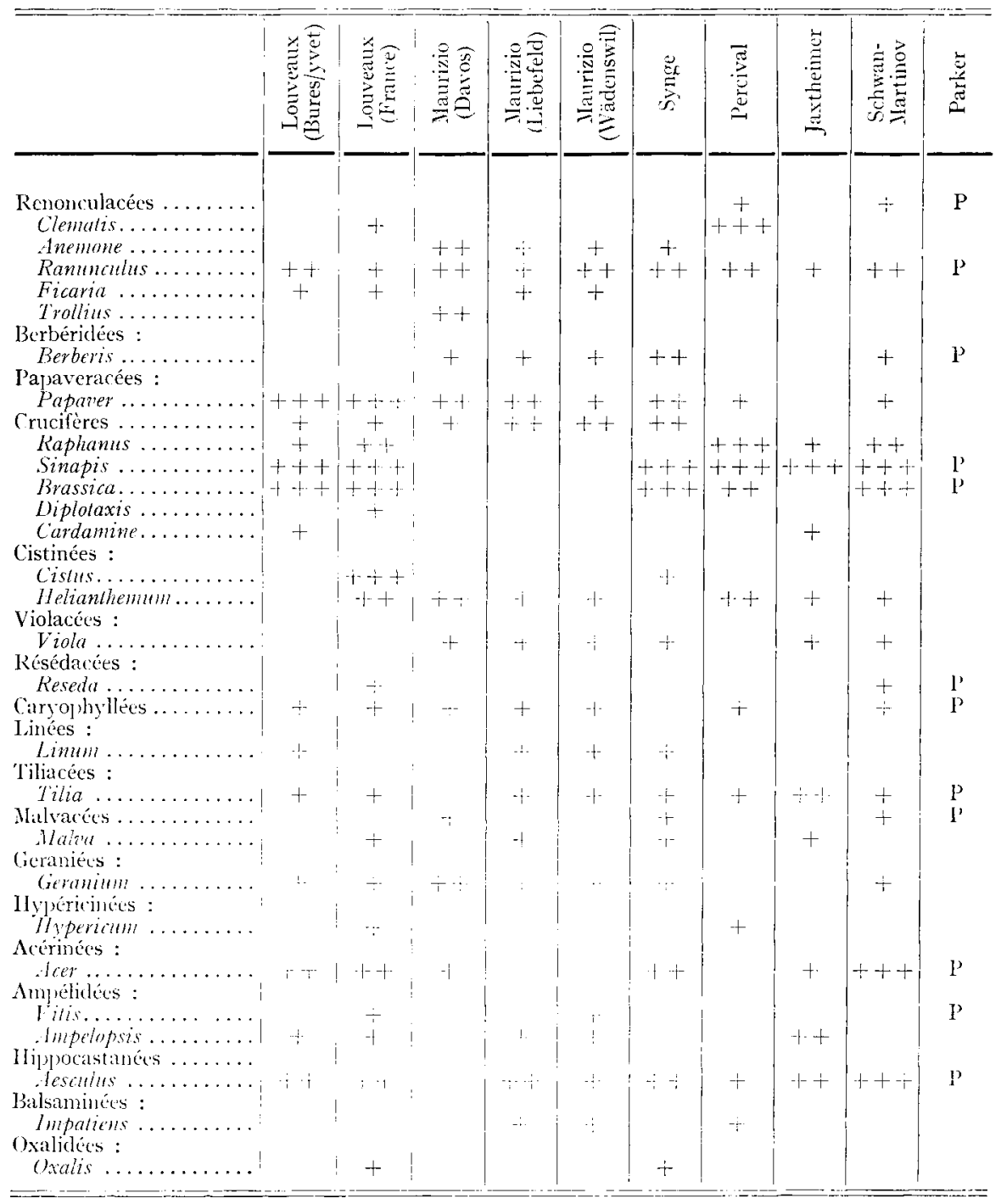


TABLEAU XXII (suite)

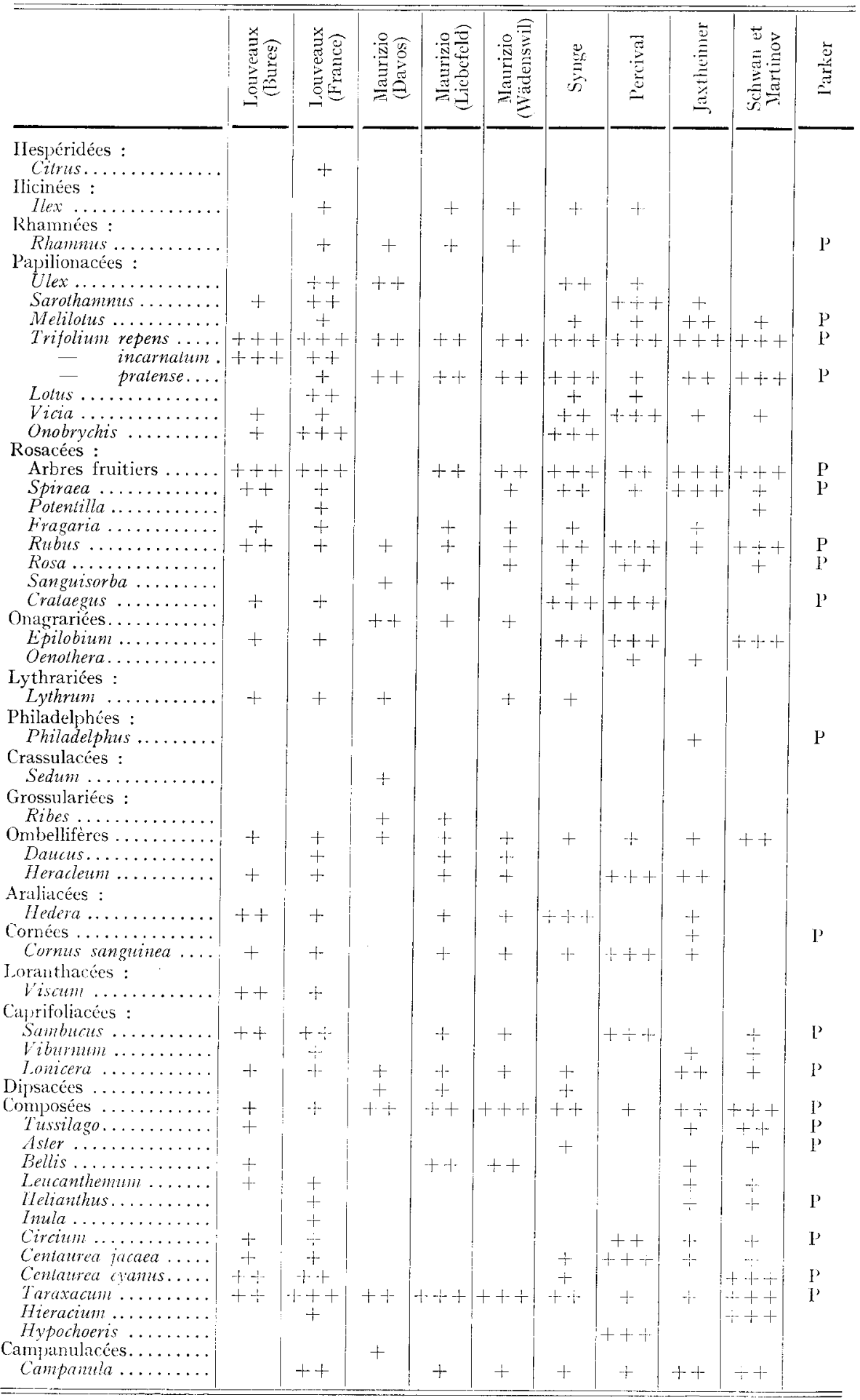


TABLEAU XXII (swite)

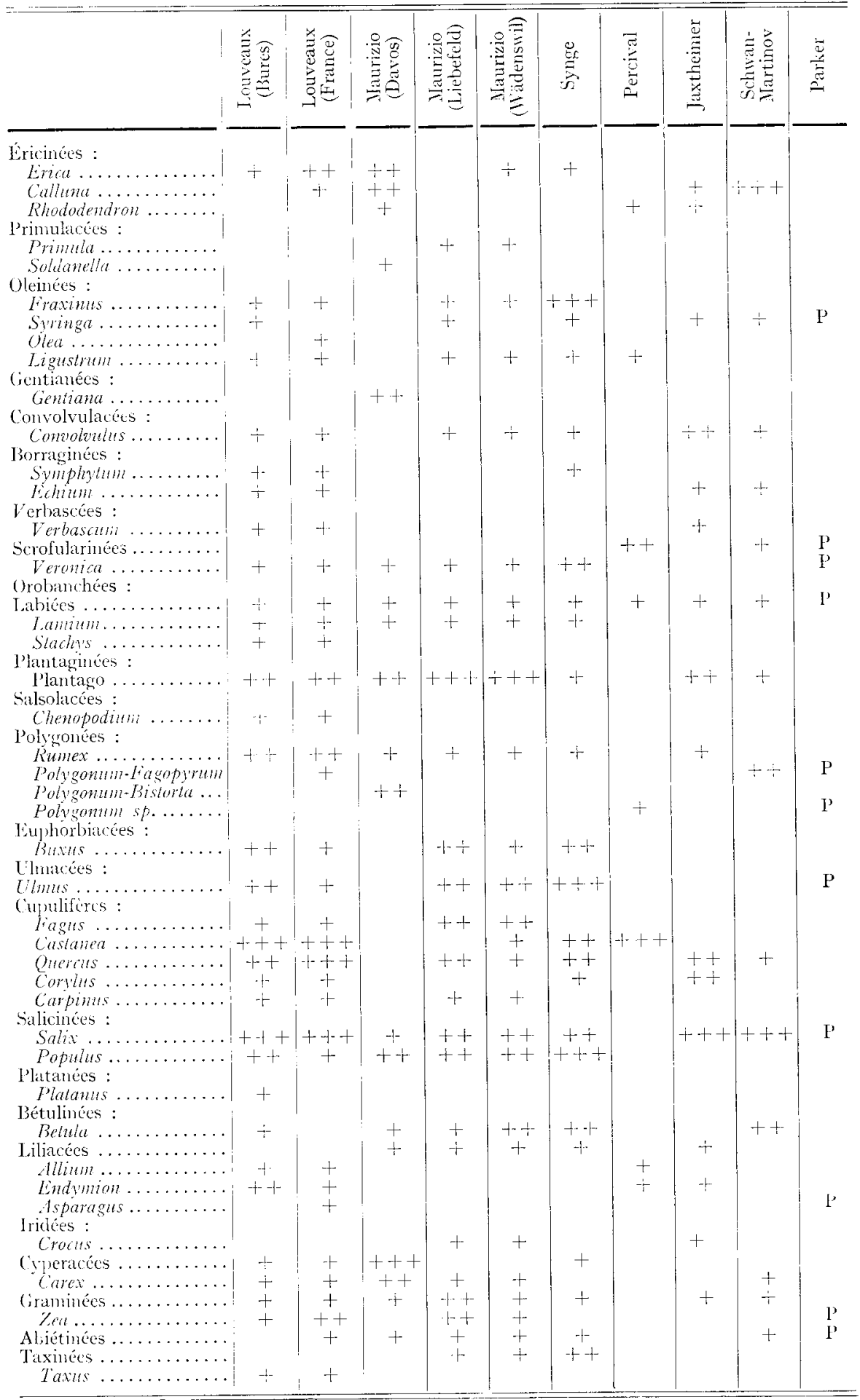


On comprend donc (que les plantes du premier printemps soient absentes de la colonne réservéc à cet auteur.

Totons encore que chaque auteur ayant calculé d'une façon dilférente limportance des pollens nous arons dil interpréter les résultats pour abontir à me codification unique de la représentation des différentes plantes pollinifères. Cette codification comprend trois degrés d'importance : $++-+-+_{-}$pollen très important, $+--=$pollen moyennement important, t. - pollen secondaire. Certaines plantes citées par différents auteurs ont été volontairement onis's; il s'agit minquement de plantes de moindre importance, très souvent horticoles et décoratives (Catalpa, Magnolia, etc.) on d'espèces qui ne sont pas caractéristiques et cont 1'utilisation par les abeilles a été visiblennent tont à fait accidentelle (Chelidonium majus par exemple). Notre tableau ne comporte que tris peu d'indications d'espèces et presque uniquenent des indications de genres on de familles. Nons avons opéré ainsi pour plus de clarté. lùn effet, au seul genre Rínunculus par exemple nous aurions dîn citer 5 on 6 espèces différentes du fait que les Renoncules dominantes à Daros ne sont pas forcément celles de Cardiff ou d'Ultuna. Ie genre Ranunculus est suffisamment homogène pour qu'on puisse, dans notre cas, préférer la notion de genre à celle d'espèce à chaque fois que la chose a un sens. I'armi les 'I'rèfles, par contre, il est utile de faire la distinction des espèces car elles acquièrent nue haute signification.

I iexamen attentif du tableau XXII nous montre un accord remarquable entre les différents auteurs en ce qui concerne les plantes essentielles. On peut dire que, pratiquement, ces plantes sont les mênes d'un bout à l'autre de l'Hurope. Ia divergence s'accentue dès que l'on passe aux plantes secondaires et aussi dès qu'on passe de la grande plaine du Nord an massif alpin. C'est I)avos pui présente les caractères floristiques les plus originaux, ce qui n'a d'ailleurs rien de surprenant.

I.a base de l'alimentation de l'abeille présente done une assez grande stabilité. Mème aux U. S. A. oì la flore est cependant fort différente de celle de l'Europe, nous retrouvons sensiblement les mémes familles et les mêmess genres dans les plantes de base. Il est intéressant de noter les substitutions qui s'opèrent d'un lieu à l'antre et qui sont très nettes chez les Composées. On peut dire que cette très vaste famille n'est que moyennement utilisée par les abeilles; cependant elle est toujours présente dans les récoltes. Taraxacum dens-leonis semble utilisé partout sur une grande échelle ainsi que les centaurées des types Jacaea et cyanus. Iin été et en automne les abeilles recherchent ce qu'on pourrait appeler vulgairement les "Composées jaunes " et sclon les lieux c'est tel ou tel genre qui est risité: Hieracinm, Hypocheris, Senecio, Solidago, Helianthus, etc. qui paraissent pouvoir se remplacer et jouer un róle semblable dans l'approvisionnement estival des abeilles. 
présente des lacunes importantes auquel cas on voit 1'utilisation massive du groupe Plantago, Rumex, Craminées, Quercus, Fagus.

Enfin l'été voit la disparition presque totale des plantes anémophiles et la dominance des plantes entomophiles, la plupart fournissant du nectar.

Nous constaterons enfin que les plantes les plus utilisées par l'abeille sont des plantes très communes, susceptibles de se présenter en peuplements denses. Mais il ne suffit pas qu'une plante soit très commune pour qu'elle soit utilisée, ni même qu'elle fournisse beaucoup de pollen. Nous arons des exemples précis avec les Conifères, les Graminées, les Cypéracées, beaucoup de Composées et bien des Papilionacées qui, quoique très communes ne foumissent que peu ou pas de pollen aux abeilles.

Si l'on veut caractériser la flore pollinifère importante pour l'abeille on dira qu'elle est essentiellement composée de plantes :

- conmunes ou trés communes,

- appartenant aux familles les plus diverses et sans qu'il soit possible d'établir une règle précise (des genres voisins peuvent être à ce sujet totalement différents),

- foumissant soit du pollen et du nectar, soit du pollen seul, sans que là $n o n$ plus une règle puisse être établie,

- dont le pollen présente pour l'abeille certaines qualités que nous ne pourons pas encore définir avec une très grande précision mais que nous savons faire intervenir la composition chimique et la caleur nutritive.

La notion de plante pollinifêre est fluctuante. - Is choix des abeilles au sein de la flore reflète vraisemblablement les besoins de la ruche qui peuvent conduire les butineuses à récolter des pollens habituellement rejetés lorsque les pollens de base que nous avons détrminés plus hant viennent à faire défaut.

I a nature offre le plus souvent un régime varié aux abeilles. Il est en effet exceptionnel qu'une récolte soit pure. Nous avons personnellement constaté dans des ruches placées au milieu de dizaines d'hectares de Colza en pleine floraison, la récolte de pollens divers que les butineuses devaient aller chercher à plusieurs centaines de mètres alors que le voisi1lage immédiat offrait du pollen en surabondance. Il est possible qu'un régime alimentaire varié soit indispensable à la santé de la ruche. MAurizIO (I944, I945) a montré que des mortalités penvent apparaître en cas de butinage à peu près exclusif sur Aesculus et sur Ranunculus puberulus Koch. On pense actuellement que la I,oque européenne pourrait bien être une maladie de carence par suite de la déficience de certains pollens; on constate en effet une disparition rapide et spontanée de la maladie par suite d'un changement de régime suivant un déplacement des ruches. 


\section{B. - LA VALEUR POLLINIFÈ DES DIFFÉRENTS ASPECTS DU TAPIS VÉGÉTAL EN FRANCE}

Si la flore utilisée par l'abeille présente une certaine homogénéité à l'échelle d'un très vaste territoire il n'en reste pas moins vrai que localement le spectre pollinique moyen peut subir des déformations plus ou moins considérables par disparition d'éléments essentiels ou par apparition d'une dominante exclusive. C'est le cas par exemple des forêts lorsqu'elles sont composées de grands arbres d'une espèce unique ou d'un nombre très réduit d'espèces (Pins, Sapins). I.e rayon d'action de l'abeille pouvant atteindre II à I2 $\mathrm{km}$ dans les cas exceptionnels et couramment 5 à $6 \mathrm{~km}$ en cas de nécessité, il faudrait supposer une forêt immense, très dense et très homogène, sans clairières ni sous-bois fleuri pour que l'abeille ne puisse pas y vivre faute de ressources en nectar et en pollen. Le cas est certainement très rare en I'rance et ce n'est qu'en certains points au cœur de la forêt vosgienne qu'on peut peut-être trouver quelques secteurs où la vie soit impossible pour les abeilles qui y seraient maintenues en permanence. I orsqu'on songe que Paris possède des ruches et qui sont prospères, on imagine facilement la quantité d'obstacles qu'il faut accumuler pour rendre impossible la vie aux abeilles dans un pays comme la lirance.

Mais entre l'impossibilité de vivre par manque de nourriture et la vie entièrement normale, il existe bien des transitions et les différents aspects du tapis végétal sont de valeur apicole très variable. Lne étude de la garrigue languedocienne dans ses rapports arec l'apiculture a été faite par RENatD (I946) de l'Ecole nationale d'Agriculture de Montpellier. Il a montré que dans l'évolution d'une friche vers le climax qui est la forêt de Chêne vert, le stade arbustif à Romarin est de loin le plus intéressant pour l'apiculture car il comporte tout un cortège de plantes de grande valetur apicole.

L'étude que nous avons faite des rapports qui existent entre l'abeille et le tapis végétal est d'un autre ordre que celle de RwnarD. Nous nous sommes servis des analyses de récoltes de pollen que nous possédons et qui portent sur l'ensemble de la lirance, pour en tirer des conclusions quant à l'utilisation du tapis régétal par l'abeille dans les différentes régions.

Chaque échantillon de pollen en soi possède peu de valcur puisqu'i1 n'est que le reflet de la flore d'un lieu bien délinité dans une certaine période de l'année. Par contre, l'accumulation d'échantillons en provenance d'une même région permet d'obtenir des chiffres moyens qui acquièrent une valeur statistique. Nous avons donc regroupé les échantillons jar affinités après avoir porté sur une carte (fig. 6I) les localités d'origine. 
Nous avons ainsi délimité des régions qui groupent des aspects divers du tapis végétal mais qui présentent malgré tout quelques caractères dominants : garrigues du Sud-Est, landes de la Gironde, terres labourables du Bassin parisien, etc.

Parallèlement à ce regroupement par régions des résultats des analyses, nous avons procédé à un regroupement des pollens identifiés en fonction de leur origine botanique et en tenant compte du biotope végétal d'origine. Nous avons ainsi défini 8 groupes de plantes pollinifères. Chacun de ces groupes correspond à un type d'utilisation du sol ou à un type de végétation particulier; ce sont:

- 1es terres labourables,

- les prairies,

- 1es herbages,

- les terres incultes,

- les forêts, bois, parcs, avenues plantées d'arbres,

- les vergers,

- les cultures florales,

- divers.

Les terres labourables nous apportent des plantes cultivées telles que les Oléagineux (Colza, Navette, Moutarde), 1e Maïs, le Sarrasin, ainsi que les plantes adventices dont les principales sont les Crucifères (Sinapis, Raphanus, Diplotaxis) ou des Composées (Centaurea cyanus et Papaver Rhaeas).

Les prairies naturelles ou artificielles sont caractérisées par une forte dominance des Légumineuses: Trèfles (T. incarnatum T. pratense), Sainfoin, Mélilots, Vesces, auxquelles il convient d'ajouter Hedysarum coronarium cultivé en Algérie.

Les herbages, qu'il est difficile de séparer nettement des prairics car les plantes qu'on y trouve sont parfois communes aux deux types d'utilisation du sol, nous apportent surtout te Trèfle blanc (T. repens), divers Lotiers dont le plus important est Lotus comiculatus, le Pissenlit (Taraxacum dens-leonis), des Centaurées dont le type est voisin de (ontaurea jacaea et enfin des Renoncules (Rammoulus repens, $R$. bulbosus, etc.).

I.es terres incultes apportent un échantillonnage très important de plantes tort diverses dont les unes sont caractéristiques de la friche, d'autres de la garrigue, d'autres de la lande, d'autres enfin des talus et bords de routes qui représentent parfois des surfaces notables. Nous citerons parmi les principales de ces plantes les Ronces (Rubus fruticosus), des Campanulacées (Campanula, Jasione), des Plantaginées (Plantago maior, $P$. lanceolata), des oseilles sauvages (Rumex sp.), plusieurs Ombellifères (Daucus, Heracleum), des Composées des types Leucanthemn et Inula (Inula viscosa en Algérie), des Vipérines (Echium vulgave, E. plantagi- 
TABleaU X

\begin{tabular}{|c|c|c|c|c|c|c|c|c|c|}
\hline & 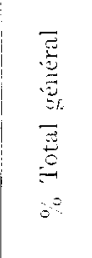 & 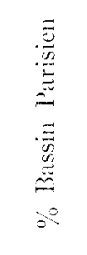 & 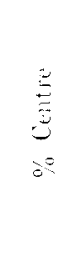 & 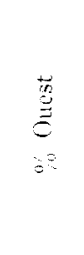 & 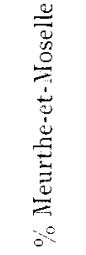 & 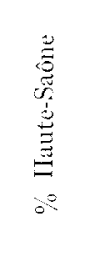 & $\frac{\ddot{3}}{3}$ & $\underset{3}{\stackrel{3}{\Xi}}$ & 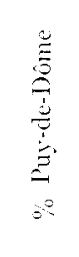 \\
\hline 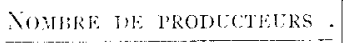 & 80 & 22 & 9 & 7 & 1 & I & I & I & $\mathbf{I}$ \\
\hline Nowibre ols eghanTlLLoxs. & 178 & 37 & 17 & 16 & 1 & I & 3 & 3 & 2 \\
\hline 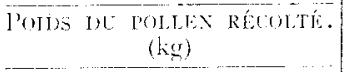 & 4539 & $\widetilde{\mathrm{r}} 145$ & 300 & 302 & 7 & I 4 & 39 & 48 & 16 \\
\hline 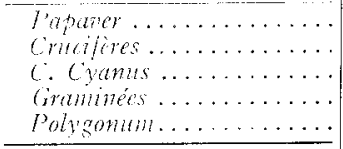 & $\begin{array}{r}0,985 \\
\text { T I,008 } \\
0,566 \\
0,488 \\
0,241 \\
\end{array}$ & $\begin{array}{r}9,37 \\
17, \mathrm{I} 4 \\
\mathrm{I}, 66 \\
0,30 \\
0,10 \\
\end{array}$ & $\begin{array}{r}19,21 \\
18,49 \\
0,56 \\
0,88 \\
0,66 \\
\end{array}$ & $\begin{array}{l}5,85 \\
6,12 \\
0,10 \\
0,54 \\
2,25\end{array}$ & $\begin{array}{c}2,00 \\
32,00 \\
- \\
1,00 \\
-\end{array}$ & $\begin{array}{c}- \\
29,00 \\
2,00 \\
2, \\
\end{array}$ & $\begin{array}{r}\mathrm{I}, \mathrm{I}, 3 \\
1.7,33 \\
0,38 \\
10,05 \\
\end{array}$ & $\begin{array}{l}22,54 \\
11,05 \\
1,04 \\
0,21 \\
\end{array}$ & $\begin{array}{c}1+, 62 \\
1,3,56 \\
\cdots \\
\cdots\end{array}$ \\
\hline TOTAT, TERRES LAMUURABLES & 19,288 & 28,57 & 39,80 & 24,86 & 35,00 & 31,00 & 38,89 & 34,89 & 28,58 \\
\hline $\begin{array}{l}\text { Trefles (incarnat et violet) } \\
\text { Sainfoin } \ldots \ldots \ldots \ldots \ldots \ldots \\
\text { Helilot } \ldots \ldots \ldots \ldots \ldots \ldots \\
\text { Hedysarm } \ldots \ldots \ldots \ldots \ldots \\
\text { Vesces } \ldots \ldots \ldots \ldots \ldots \ldots \\
\end{array}$ & $\begin{array}{l}1,416 \\
9,386 \\
0,827 \\
0,049 \\
0,067\end{array}$ & $\begin{array}{r}\mathrm{I}, 75 \\
2 \mathrm{I}, 37 \\
0,42 \\
-0,06\end{array}$ & $\begin{array}{l}2,42 \\
6,3^{5} \\
-- \\
- \\
-\end{array}$ & $\begin{array}{l}6,48 \\
2,74 \\
-- \\
\cdots- \\
0,13 \\
\end{array}$ & $\begin{array}{l}-- \\
27,00 \\
- \\
- \\
1,00\end{array}$ & $\begin{array}{l}1, \infty 0 \\
-- \\
-- \\
- \\
\end{array}$ & $\begin{array}{l}- \\
19,26 \\
- \\
- \\
\end{array}$ & $\begin{array}{r}2,21 \\
15,04 \\
\cdots \\
-- \\
\end{array}$ & $\begin{array}{r}8,94 \\
24,37 \\
- \\
- \\
-\end{array}$ \\
\hline TOTAL PRAERIES $\ldots \ldots \ldots \ldots$ & $I I, 745$ & $2,3,60$ & 8,78 & 9,35 & 28,00 & 1,00 & 19,26 & 17,25 & $3,3,3 \mathbf{r}$ \\
\hline 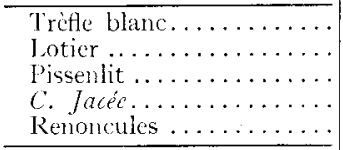 & $\begin{array}{l}3,602 \\
0,869 \\
3,469 \\
0,163 \\
0,2,36 \\
\end{array}$ & $\begin{array}{l}3,3 \mathrm{I} \\
0,33 \\
5,00 \\
0,10 \\
0,21\end{array}$ & $\begin{array}{l}2,23 \\
0,98 \\
2,42 \\
0,40\end{array}$ & $\begin{array}{r}29,64 \\
8,21 \\
2,66 \\
0,68 \\
0,84 \\
\end{array}$ & $\begin{array}{l}6,00 \\
3,00 \\
1,00 \\
\end{array}$ & $\begin{array}{c}- \\
- \\
13,00 \\
-\cdot \\
\end{array}$ & $\begin{array}{l}4,26 \\
3,44 \\
0.18 \\
0 .- \\
\end{array}$ & $\begin{array}{l}0,2 \mathrm{I} \\
6,92 \\
0,40 \\
-\end{array}$ & $\begin{array}{l}1,6,3 \\
\frac{1}{0,75} \\
-\overline{-81} \\
2,81\end{array}$ \\
\hline TOTAL HERRAGES $\ldots \ldots \ldots$ & 8.339 & 8,95 & $0,0,3$ & 42,03 & 10,00 & $1,3,00$ & 7,88 & 7,53 & 5,19 \\
\hline 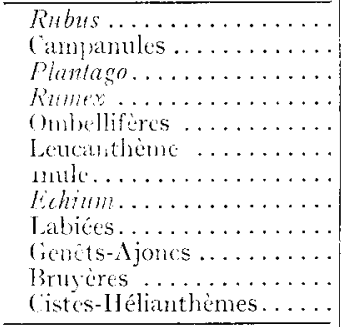 & $\begin{array}{l}2,250 \\
0,935 \\
1,192 \\
1,456 \\
0,326 \\
0,279 \\
0,085 \\
0,292 \\
0,240 \\
4,064 \\
4,719 \\
18,036 \\
\end{array}$ & $\begin{array}{l}1,25 \\
1,06 \\
1,71 \\
3,72 \\
0,24 \\
0,48 \\
-, \\
- \\
0,02 \\
0,57 \\
0,09 \\
0,08 \\
\end{array}$ & $\begin{array}{l}4,02 \\
3,76 \\
0,78 \\
0,61 \\
0,87 \\
0,36 \\
- \\
- \\
0,07 \\
2,24 \\
0,02 \\
\end{array}$ & $\begin{array}{l}4,66 \\
0,07 \\
1,63 \\
0,79 \\
1,1,3 \\
\mathrm{~T}, 27 \\
\cdots \\
- \\
0,04 \\
3,07 \\
0,09 \\
-\end{array}$ & $\begin{array}{c}\cdots- \\
\cdots, \\
17,00 \\
- \\
-- \\
\mathrm{T}, \infty \\
\cdots \\
- \\
- \\
\cdots \\
- \\
-\end{array}$ & $\begin{array}{c}- \\
12,00 \\
1,00 \\
-\cdots \\
2, \infty \\
- \\
-- \\
- \\
\cdots \\
\cdots \\
-\end{array}$ & $\begin{array}{c}20,00 \\
-\ldots \\
- \\
-- \\
-- \\
- \\
\cdots \\
\cdots \\
11,87 \\
0,43 \\
\cdots \\
\cdots\end{array}$ & $\begin{array}{l}3, \mathrm{r} \\
\cdots- \\
\mathrm{T}, 25 \\
1,19 \\
4,17 \\
0,7 \mathrm{~T} \\
\cdots- \\
\cdots- \\
- \\
-- \\
0,62 \\
- \\
\end{array}$ & $\begin{array}{l}0,8 \mathrm{I} \\
0,8 \mathrm{I} \\
-- \\
7,3 \mathrm{r} \\
- \\
0,37 \\
0,3 \mathrm{I} \\
- \\
\end{array}$ \\
\hline TOTAI TERRISS INCULTES ... & 33,874 & 9,22 & $1,3,03$ & 12,75 & 18,00 & 15.00 & 32,30 & I I, I I & 10,11 \\
\hline 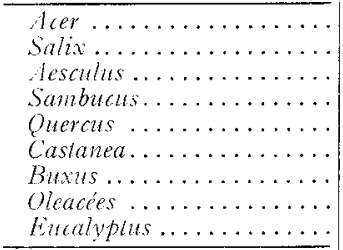 & $\begin{array}{l}0,164 \\
5,763 \\
0,285 \\
1,541 \\
1,075 \\
5,804 \\
0,885 \\
0,837 \\
0,850\end{array}$ & $\begin{array}{r}0,4 \mathrm{I} \\
\mathrm{1} 0,26 \\
0,79 \\
4,02 \\
0,37 \\
2,40 \\
\cdots \\
\cdots\end{array}$ & $\begin{array}{l}0,36 \\
5,31 \\
0,60 \\
2,80 \\
0,03 \\
5,39 \\
0,18 \\
-- \\
--\end{array}$ & $\begin{array}{l}0,34 \\
0,30 \\
- \\
0,24 \\
0,78 \\
4,86 \\
\end{array}$ & $\begin{array}{c}-\cdots \\
--\cdot \\
\cdots- \\
\cdots- \\
\cdots- \\
\cdots- \\
\cdots \\
\cdots \\
\cdots\end{array}$ & $\begin{array}{c}3,00 \\
\mathrm{I} 1,00 \\
- \\
3,00 \\
\cdots- \\
\cdots \\
-- \\
- \\
\end{array}$ & $\begin{array}{l}- \\
-- \\
-- \\
0,79 \\
- \\
- \\
- \\
-\end{array}$ & $\begin{array}{c}- \\
- \\
\cdots \\
1,3,44 \\
- \\
11,48 \\
\cdots- \\
\cdots- \\
\cdots-\end{array}$ & $\begin{array}{l}\cdots, 19 \\
6,19 \\
- \\
- \\
- \\
- \\
\mathrm{I}, 3 \mathrm{I} \\
\cdots-\end{array}$ \\
\hline TOTAL BOIS, FORETS, l'ARCS. & 17,204 & 18,25 & $\mathrm{~T} 4,67$ & $6,+2$ & 0 & 17,00 & 0,79 & 24,92 & 7,50 \\
\hline $\begin{array}{l}\text { Arbres fruitiers . . . . . . } \\
\text { Cilmas .............. }\end{array}$ & $\begin{array}{l}4,187 \\
0, \text { II I I } \\
\end{array}$ & $9,5,3$ & $\begin{array}{l}7,38 \\
-\end{array}$ & 4,82 & 8,00 & 19,00 & - & $\begin{array}{l}2,37 \\
\ldots \ldots .\end{array}$ & 7,94 \\
\hline TOTAL VERGERS $\ldots \ldots \ldots \ldots$ & 4.298 & $9,5,3$ & 7,38 & 4,82 & 8,00 & $\mathrm{~T} 9,00$ & 0 & 2,37 & 7,94 \\
\hline Mimosat $\ldots \ldots \ldots \ldots \ldots$ & 0,307 & - & $\cdots-$ & $\cdots$ & $-\cdots$ & $\cdots$ & $\cdots$ & - & 二 \\
\hline TOTAI CelTuRES FloRAILS.. & 0,307 & $\cdots$ & +-- & $\cdots$ & $\cdot-$ & - & 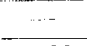 & $-\cdots$ & - \\
\hline Divers $\ldots \ldots \ldots \ldots \ldots$ & 4,945 & $\mathrm{I}, 88$ & $\mathrm{IO}, 3 \mathrm{I}$ & 0,23 & 1,00 & $4, \infty$ & 0,88 & 1,93 & 7,77 \\
\hline
\end{tabular}


(I, I959) RECHERCHISS SUR LA RÉCOLTI DU POLLEN

\section{née 1950}

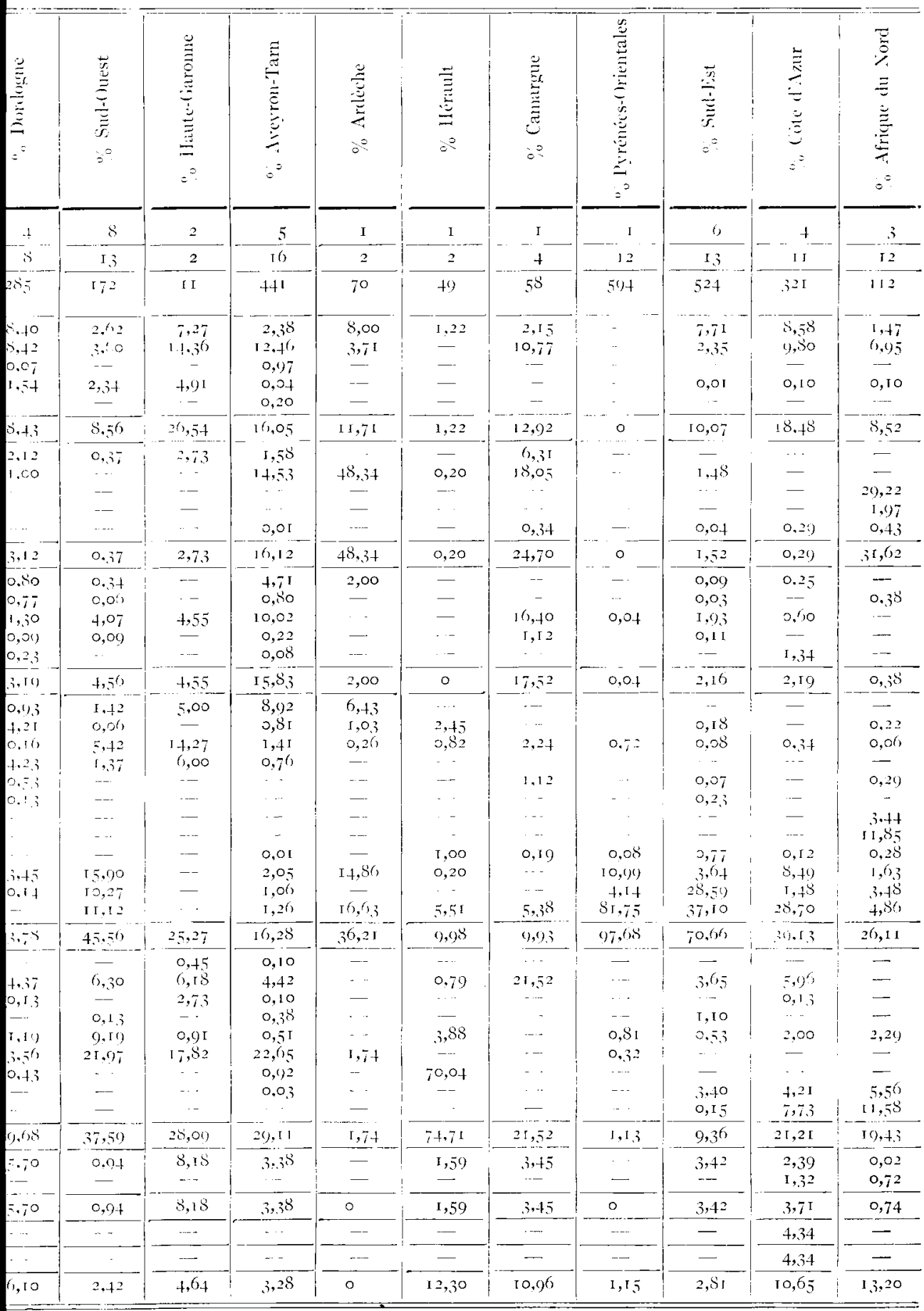

Amales des Abeilles, - ro59. 
TABLEAU XX

\begin{tabular}{|c|c|c|c|c|c|c|c|c|c|c|c|}
\hline & \multicolumn{2}{|c|}{ Bassin-Parisien } & \multicolumn{2}{|c|}{$\begin{array}{c}\text { Centre } \\
\text { (L.-et-C.) }\end{array}$} & \multicolumn{2}{|c|}{ Ouest } & \multicolumn{2}{|c|}{$\begin{array}{c}\text { Sud-Ouest I } \\
\text { Vendée-Charente }\end{array}$} & \multicolumn{2}{|c|}{$\begin{array}{c}\text { Sud-Ouest II } \\
\text { Gironde-Landes }\end{array}$} & \\
\hline & $\mathrm{kg}$ & $\%$ & $\mathrm{~kg}$ & $\%$ & $\operatorname{lig}$ & $\%$ & $\mathrm{~kg}$ & $\%$ & $\mathrm{~kg}$ & $\%$ & \\
\hline $\begin{array}{l}\text { NongRE } \text { de produc- } \\
\text { TEURS } \ldots \ldots \ldots \ldots \ldots \\
\end{array}$ & I I & & I & & $\therefore$ & & 2 & & 2 & & \\
\hline $\begin{array}{l}\text { Yonbre des échantiL- } \\
\text { LONs } . \cdots \cdots \cdots \cdots \cdots \cdots \\
\end{array}$ & 22 & & I & & I4 & & 5 & & 3 & & \\
\hline $\begin{array}{l}\text { POIDS TOTAL PAR RÉ- } \\
\text { GION } \ldots \ldots \ldots \ldots \ldots \\
\end{array}$ & $2 \circ 45$ & & 85,5 & & 605 & & 69 & & 79 & & \\
\hline 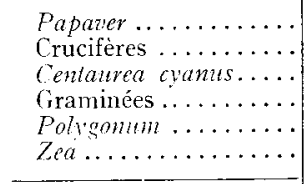 & $\begin{array}{r}3,3,7 \\
9 \mathrm{II,1} \\
25,8\end{array}$ & $\begin{array}{r}\mathrm{T}, 64 \\
44,53 \\
\mathrm{I}, 26\end{array}$ & $\begin{array}{r}1,7 \\
35,9\end{array}$ & $\begin{array}{r}2 \\
12\end{array}$ & $\begin{array}{r}24,6 \\
47,8 \\
0,2 \\
6,2\end{array}$ & $\begin{array}{l}4,06 \\
7,90 \\
0,03 \\
1,02\end{array}$ & $\begin{array}{r}8,8 \\
23,4 \\
0,3 \\
0,3 \\
0,3\end{array}$ & $\begin{array}{r}12,75 \\
33,90 \\
\\
0,43 \\
0,43 \\
0,43 \\
\end{array}$ & $\begin{array}{r}14,4 \\
6,3\end{array}$ & $\begin{array}{r}18,22 \\
7,97\end{array}$ & \\
\hline $\begin{array}{r}\text { TOTAL TERRFS } \text { LABOU } \\
\text { RARLES } \cdots \cdots \cdots \cdots \cdots \cdots \\
\end{array}$ & 970,6 & 47,43 & 37,6 & 14 & 78,8 & $\mathrm{I}, 3,01$ & $33, \mathrm{x}$ & 47,94 & 20,7 & 26,19 & \\
\hline $\begin{array}{l}\text { Trefle incamat et } \\
\quad \text { Trüfe violet } \ldots \ldots \\
\text { Sainfoin } \ldots \ldots \ldots \ldots \ldots \ldots \\
\text { Melelilot } \ldots \ldots \ldots \ldots \ldots \ldots \ldots \\
\text { Vesces } \ldots \ldots \ldots \ldots \ldots \ldots\end{array}$ & $\begin{array}{r}2,5 \\
542 \\
0,6 \\
0,3\end{array}$ & $\begin{array}{r}0,12 \\
26,50 \\
0,02 \\
0,01\end{array}$ & & & $\begin{array}{r}28,9 \\
4,4 \\
0,1\end{array}$ & $\begin{array}{l}4,76 \\
0,72 \\
0,01\end{array}$ & $\begin{array}{l}0,4 \\
\mathrm{r}, 7\end{array}$ & $\begin{array}{l}0,57 \\
2,4^{6}\end{array}$ & 4,8 & 6,07 & \\
\hline TOTAL PRAIRIEs......... & 545,4 & 26,65 & & & 33,4 & 5,49 & $2, \mathrm{I}$ & 3,03 & 4,8 & 6,07 & \\
\hline 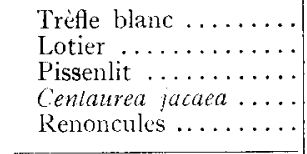 & $\begin{array}{r}3 \mathrm{I}, 9 \\
7,9 \\
10,1 \\
0,7\end{array}$ & $\begin{array}{l}I, 5,5 \\
0,38 \\
0,49 \\
0,03\end{array}$ & & & $\begin{array}{r}54,6 \\
0,4 \\
57,2 \\
2,0 \\
0,3\end{array}$ & $\begin{array}{l}9,02 \\
0,06 \\
9,45 \\
0,33 \\
0,04\end{array}$ & $\mathrm{I} 2,3$ & 17,82 & $\begin{array}{l}0,6 \\
2,1 \\
0,6 \\
0,4\end{array}$ & $\begin{array}{l}0,75 \\
2,65 \\
0,75 \\
0,50\end{array}$ & \\
\hline TOTAL HERBAGES $\ldots . .$. & 50,6 & 2,45 & & & $\mathrm{II} 4,5$ & 18,90 & 12,3 & 17,82 & $3, \bar{i}$ & 4,65 & \\
\hline 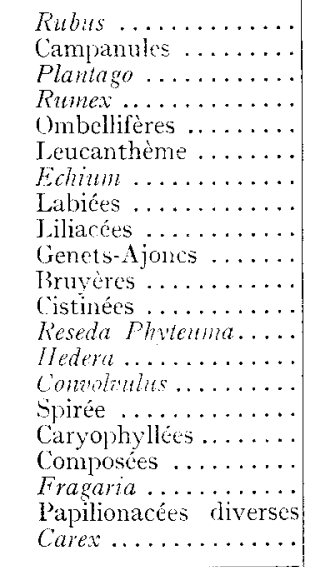 & $\begin{array}{r}0,1 \\
15,3 \\
53,3 \\
1,3 \\
1,7 \\
0,2 \\
3,1\end{array}$ & $\begin{array}{l}0,01 \\
0,74 \\
2,60 \\
0,06 \\
0,08 \\
0,01 \\
0,14\end{array}$ & $\begin{array}{r}2,6 \\
2,6 \\
25,6 \\
0,8\end{array}$ & $\begin{array}{r}3 \\
30 \\
30 \\
1\end{array}$ & $\begin{array}{l}2,3 \\
1,4 \\
0,2 \\
6,6 \\
1,5\end{array}$ & $\begin{array}{l}0,38 \\
0,23 \\
0,09 \\
1,08 \\
0,24\end{array}$ & $\begin{array}{l}0, \mathrm{I} \\
0,4 \\
0, \mathrm{~T}\end{array}$ & $\begin{array}{l}0, I_{4} \\
0,57 \\
0, I_{4}\end{array}$ & $\begin{array}{l}0,7 \\
0,7 \\
3,2 \\
0,4\end{array}$ & $\begin{array}{l}0,88 \\
0,88 \\
4,0,5 \\
0,50\end{array}$ & \\
\hline $\begin{array}{c}\text { TOTAL TERRES MNCLL } \\
\text { TES } \ldots \ldots \ldots \ldots \ldots \ldots \ldots\end{array}$ & I 20,8 & 5,86 & $3^{6,8}$ & 43 & \begin{tabular}{l|l} 
& $\mathrm{I} 3,8$ \\
\end{tabular} & 2,24 & I 5,7 & $22,7 \mathrm{I}$ & 5,2 & $6,5^{6}$ & \\
\hline
\end{tabular}




\section{Année 1950}

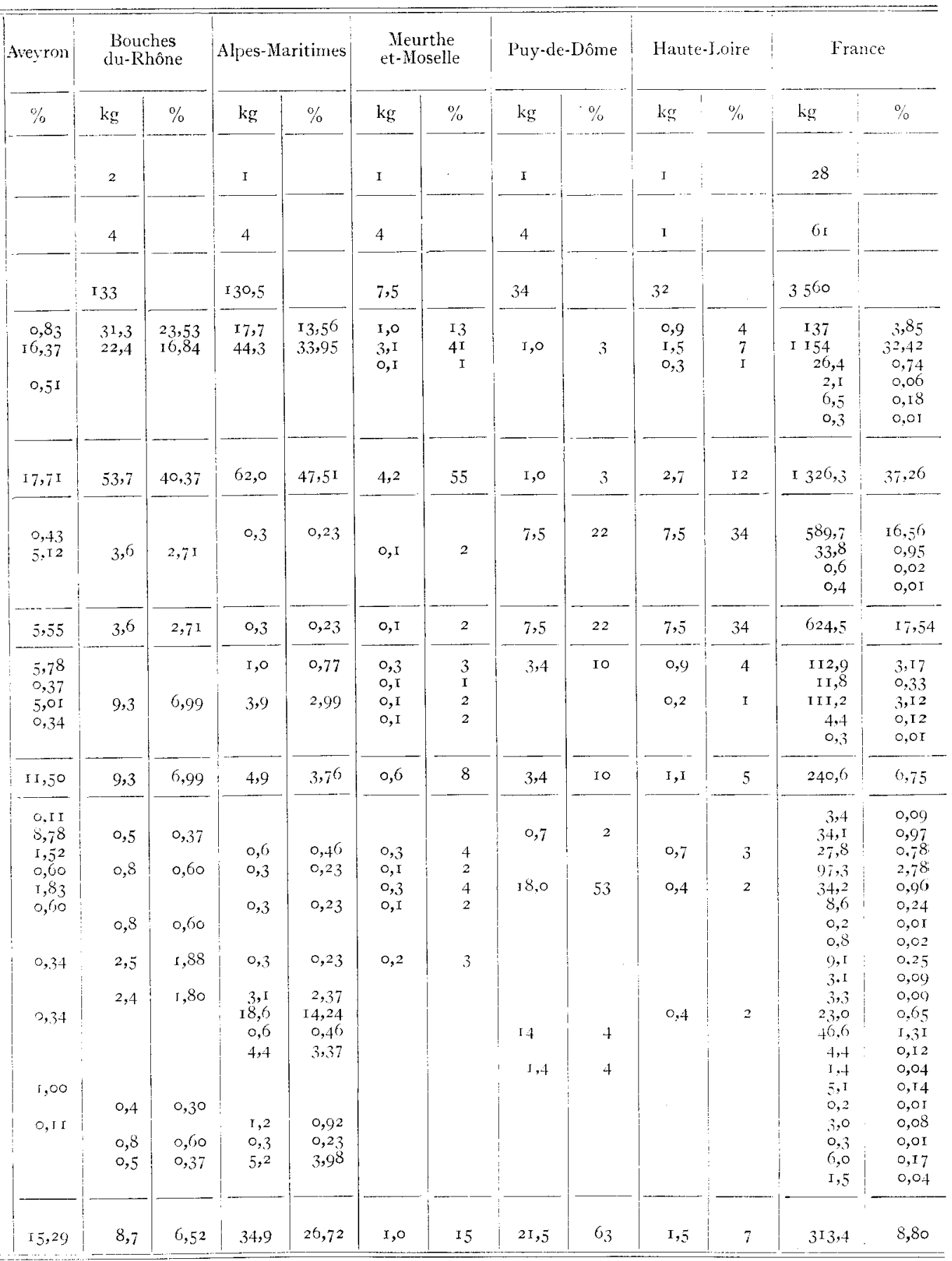


TABLEAU XXIV

\begin{tabular}{|c|c|c|c|c|c|c|c|c|c|c|c|}
\hline & \multicolumn{2}{|c|}{ Bassin-Parisien } & \multicolumn{2}{|c|}{$\begin{array}{l}\text { Centre } \\
\text { (L.-et-C.) }\end{array}$} & \multicolumn{2}{|c|}{ Ouest } & \multicolumn{2}{|c|}{$\begin{array}{c}\text { Sud-Ouest I } \\
\text { Vendée-Charente }\end{array}$} & \multicolumn{2}{|c|}{$\begin{array}{c}\text { Sud-Ouest II } \\
\text { Gironde-Landes }\end{array}$} & \multirow{2}{*}{$\frac{\text { Aveyron }}{k \mathrm{~kg}}$} \\
\hline & $\mathrm{kg}$ & $\%$ & $\mathrm{~kg}$ & $\%$ & $\mathrm{~kg}$ & $\%$ & $\mathrm{~kg}$ & $\%$ & $\mathrm{~kg}$ & $\%$ & \\
\hline 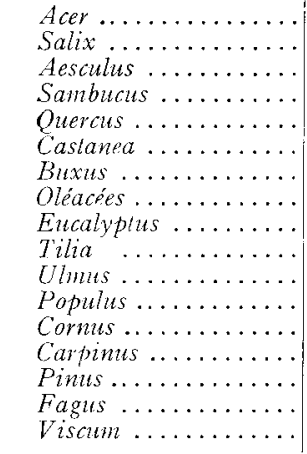 & $\begin{array}{r}43,6 \\
55,5 \\
8,0 \\
0,8 \\
79,6 \\
5,7\end{array}$ & $\begin{array}{l}2,13 \\
2,71 \\
0,39 \\
0,03 \\
3,89 \\
0,27\end{array}$ & $\begin{array}{l}0,9 \\
0,8 \\
6,0 \\
1,7\end{array}$ & $\begin{array}{l}T \\
T \\
T \\
2\end{array}$ & $\begin{array}{r}0,7 \\
39,7 \\
0,2 \\
6,3 \\
68,0\end{array}$ & $\begin{array}{r}0, \mathrm{II} \\
6,56 \\
0,0,3 \\
\mathrm{I}, 04 \\
\mathrm{I}, 23\end{array}$ & $\begin{array}{l}0,7 \\
0,1 \\
1,2 \\
\text { J,8 }\end{array}$ & $\begin{array}{l}\mathrm{T}, \mathrm{OI} \\
0, \mathrm{~T} 4 \\
\mathrm{I}, 73 \\
2,60\end{array}$ & $\begin{array}{r}2,8 \\
0,2 \\
1,8 \\
4,8 \\
4,8 \\
25,8\end{array}$ & $\begin{array}{r}3,54 \\
0,25 \\
2,27 \\
6,07 \\
23,65\end{array}$ & $\begin{array}{r}0,6 \\
0,3 \\
59,7 \\
85,3\end{array}$ \\
\hline $\begin{array}{r}\text { TOTAI FORÊTS, BOIS, } \\
\text { PARC. } \\
\end{array}$ & 202 & 9,83 & 9,4 & II & 163,1 & ${ }^{19} 9,20$ & $5, \mathrm{I}$ & 7,35 & $3^{6, \mathrm{I}}$ & 45,66 & 150,6 \\
\hline 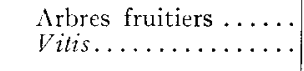 & 146,4 & 7,15 & & & 274,4 & 40,89 & 0,5 & 0,72 & 7,7 & 9,74 & $\begin{array}{r}I, 3,4 \\
5,9\end{array}$ \\
\hline Total vergers $\ldots \ldots \ldots$ & 146,4 & 7,15 & & & 274,4 & 40,89 & 0,5 & 0,72 & 7,7 & 9,74 & $\mathrm{I9,3}$ \\
\hline $\begin{array}{l}\text { Minosa } \ldots \ldots \ldots \ldots \ldots \\
\text { Divers .................... }\end{array}$ & 9,0 & 0,63 & $\mathrm{I}, 7$ & 2 & 0,5 & 0,23 & 0,2 & $0,4,3$ & 0,8 & 1,13 & 4,6 \\
\hline
\end{tabular}

neum), quelques rares Iabiées (Lamium, Stachys), des genêts et ajoncs (Ulex sp. Sarothamnus scoparius), des Bruyères (Erica arborea, Calluna vulgaris), des Cistinées (Cistus albidus, C. monspeliensis, C. salviaefolius), Reseda phyteuma, Hedera helix, Convolvulus sp., Spiraea ulmaria, Carex sp., etc.

I es arbres et arbustes des forêts, bois, parcs, avenues etc. sont principalement des Erables (Acer pseudo-platanus), des Saules (Salix sp.), diverses Oléacées (Ligustrum vulgare, Syringa vulgaris), des Chênes (Quercus sp.), Hêtres (Fagus silvatica), Charmes (Carpinus betulus), Peupliers (Populus sp.), 'Tilleuls (Tilia platyphyllos), Cornouillers (Cormus sanguinea), Ormes (Ulmus campestris), Châtaigniers (Castanea vulgaris), Marronniers (Aesculus sp.), Eucalyptus (dans le Midi de la France), Sambucus sp., Sambucus sp., Buxus sempervirens, Viscum album et enfin quelques rares Conifères (Pinus sp.).

Les arbres fruitiers comprennent à peu près tout ce qui est habituellement cultivé dans nos vergers : Cerisiers, Pruniers, Poiriers, Pommiers, Pêchers, Abricotiers, Amandiers, etc. Nous avons joint, un peu arbitrairement la Vigne aux arbres fruitiers; le pollen de Vitis n'est qu'une ressource très secondaire pour les abeilles et on ne le trouve dans les récoltes que dans le Midi. 
- Calcul du poids de chaque constituant dans le lot de pollen en partant des pourcentages déterminés.

- Addition des poids de chaque constituant pour l'ensemble des lots de pollen d'une région.

- Calcul du poids total de pollen récolté par région.

- Calcul du pourcentage moyen des différents constituants pour chaque région.

- Calcul dı pourcentage noyen des différents constituants pour l'ensimble de la lirance.

Ie tableau XXV permet de comparer les résultats des deux années I955 et I956 et de suivre ainsi l'évolution de 1'utilisation de la flore en fonction de divers facteurs, principalenent météorologiques.

Le point intéressant est la confrontation du spectre pollinique obtenu pour chaque région avec les caractères botaniques et agricoles desdites régions. I a confrontation est d'autant plus fructueuse que la récolte analysée est plus abondante et répartie sur un grand nombre d'échantillons. Nous arous utilisé comme documents de référence les cartes de la végétation au $\mathrm{I} / 200000^{\mathrm{e}}$, la carte du tapis végétal de la lirance de CiAcssion ainsi (qu'un document agronomique important, "I a localisation des cultures et des productions animales en France " de KLA'TzMAN (I955). Les corrélations entre ce que nous savons du tapis végétal et de 1'utilisation du sol d'une part, la récolte du pollen d'autre part, est satisfaisante. Prenons quelques exemples :

Io Le Bassin parisien. - I,e Bassin parisien peut être caractérisé dans son ensemble conme une région très agricole où le territoire est occupé essentiellement par les terres labourables. Peu d'herbages, prairies: artificielles nombreuses oì dominent le Sainfoin et la I,uzerne. I,es terres incultes sont constituées surtout par les talus et bords des chemins; peu ou pas de landes. I,es forêts sont relativement nombreuses et importantes. Ise Chêne pédonculé et le Hêtre y dominent ; le Châtaignier est abondant. Nous retrouvons dans les récoltes de pollen toutes ces caractéristiques. I,e poste " terres labourables" est le plus important surtout en $195^{\circ}$ où les Crucifères furent favorisées par 1'humidité. Par contre Paparer fut plus abondant en I955 qui fut plus ensoleillé. I,es postes " herbages " et "terres incultes " sont mal représentés, tandis (que dans les prairies le Sainfoin montre sa nette supériorité sur les 'Trèfles. La forêt arrive en $3^{\mathrm{e}}$ position dans les deux années. I,es vergers ne sont guère plus importants que les terres incultes.

$2^{\circ}$ Région Onest. - I es années 1955 et I956 présentent de notables différences qui sont dues au fait que $195^{6}$ a vu une utilisation beaucoup) plus massive du pollen des arbres fruitiers surtout Pommiers et Poiriers. I a place des herbages est prédominante en I95.5. I, équilibre entre les 
(I, I959) RECHERCHFS SUR LA RÉCOLTE DU POLLEN

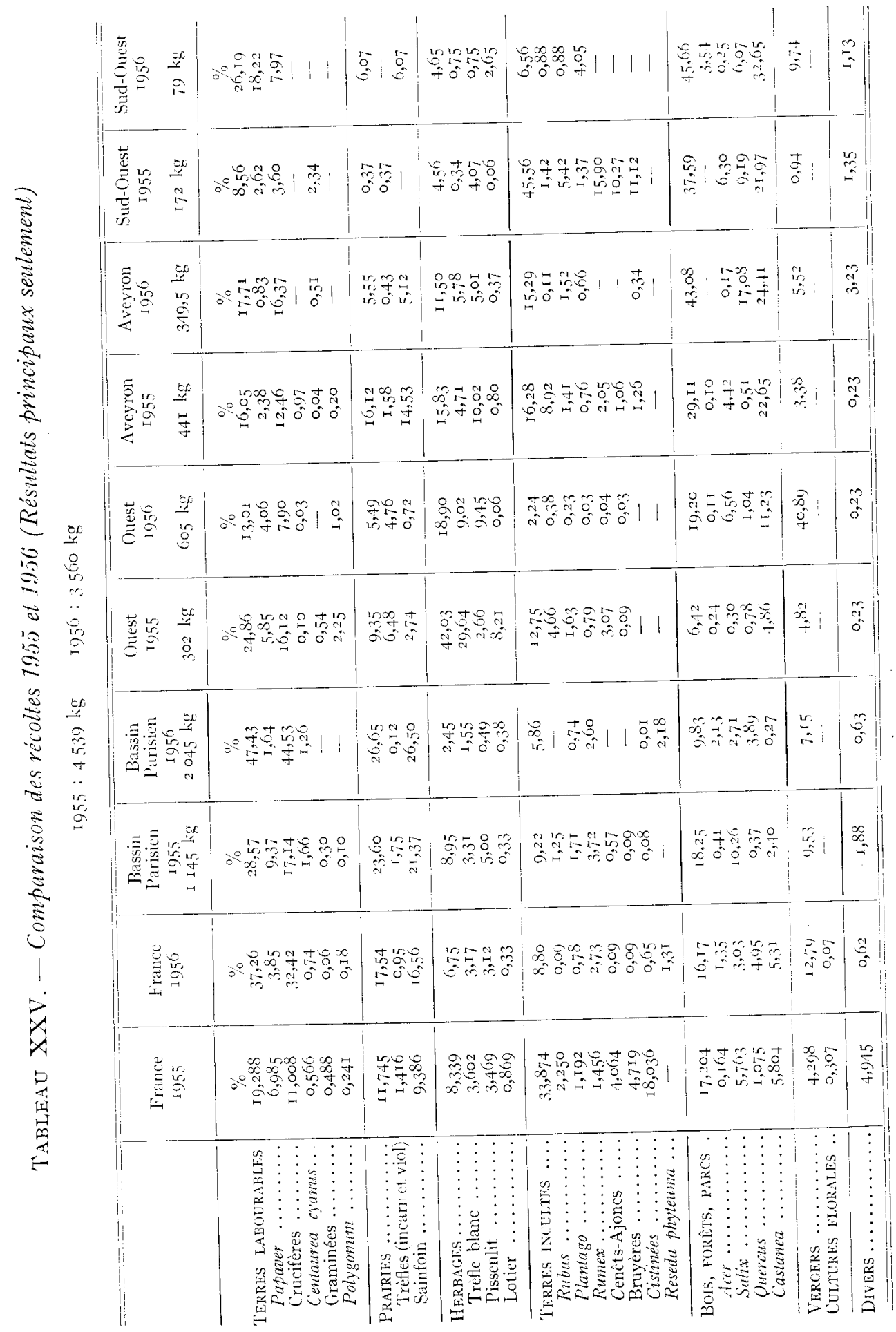


différents postes reflète bien le pays de boccages et de polyculture avec une importante part réservée au pâturage. I,e Châtaignier est encore abondant dans les haies et les bosquets. I,e Sarrasin fait son apparition avec des pourcentages notables malgré la très forte régression de cette culture.

$3^{0}$ Aveyron. - Pour cette région aussi la récolte du pollen reflète avec précision les activités agricoles et les caractères botaniques. Le Chàtaignier occupe une place maîtresse et donne le quart environ du pollen récolté. Pour les autres postes il y a un bon équilibre entre les cultures, les prairies, les herbages et les terres incultes. Nous avons affaire, là encore, à une région paurre oì domine la polyculture et oì le boisement est assez important ainsi que les terres incultes.

$4^{\circ}$ Sud-Onest. - La faible récolte de $195^{6}$, qui de plus est nal répartie, antorise à peine la comparaison des deux années. Il nous semble que I955 est beaucoup plus caractéristique avec ses 45 p. Ioo de pollen en provenance de la lande : Ajoncs, Genêts, Bruyères, Cistus salizacfolizs. I a forêt représente, là aussi une source de pollen importante mais le genre Pinus, malgré sa très grande abondance, n'est pas représenté, ce qui corrobore ce que nous savons déjà du pollen des Conifères. C'est le Châtaignier, fort abondant d'ailleurs en dehors des plantations de Pins maritimes qui domine. I,es postes relevant de l'agriculture sont d'importance très secondaire.

$5^{\circ}$ Autres régions. - I es gelées de février 1956 ayant détruit une partie de la végétation arbustive du Midi méditerranéen nous constatons une défection massive des producteurs de pollen de cette région en 1956 . Seule l'année I 955 peut nous fournir des indications valables. Ires Pyrénées orientales nous ont apporté avec les garrigues à Ciste de Montpellier une nusse énorme et presque pure de pollen de Cistinées. Tout le sud-Est est d'ailleurs caractérisé par (istus, Erica arborea, Clex parifloms et autres plantes du groule des genêts et ajoncs. In reportant sur la carte de la régétation des Pyrénées orientales de GArssen l'emplacement des ruchers producteurs de pollen de Ciste nous avons pu constater l'étommante concordance des indications de la carte at de celles des récoltes de pollen. I es ruches placées sur la garrigue à Cistes ont récolté le pollen de cette plante pour plus de so p). Ioo. I.es pollens secondaites sont la Bruyère, les crencts et Ajoncs, le Chêne vert et un peu de Châtaignier; aucunc trace du pollen de plantes cultivées.

La Côte d'Azur nous offre, à côté de la flore habituelle du sud-Isst quelques plantes bien particulières et qu'on ne saurait pratiquement trouver ailleurs en France: Mimosa, Eucalyptus Citrus et quelques plantes exotiques qui n'ont d'ailleurs pas été déterminées avec précision.

Pour plus de détails nous renvoyons aux différents tableaux récapitulatifs qui sont particulièrement parlants. 


\section{C. - INCIDENCES AGRONOMIQUES ET AUTRES DU PROBLÈME DE LA RÉCOLTE DU POLLEN PAR LES ABEILLES}

Eùn dehors des problèmes de pollinisation des plantes cultivées que nous ne pouvons aborder ici, la récolte du pollen par les abeilles pose plusieurs problèmes fort intéressants.

Nous venons de reconnaitre que les différents types d'utilisation du sol et les différents aspects du tapis végétal conduisent à des formules polliniques de récoltes fort variées. ('énéralement, en un lieu donné les abeilles pourront utiliser à la fois le pollen d'une lande, d'une forêt, d'une zone cultirée, etc., ce qui permettra une bonne répartition dans le temps des ressources polliniques disponibles. Mais il arrive, et c'est le cas dans la garrigue on dans la forêt landaise que les ressources en pollen, quoique abondantes, se trourent très inégalement réparties dans le temps. (Que devient la ruche soumise à ce genre de réginle ? C'est un problène que nous pensons deroir mériter une étude, d'antant plus que nous savons que les pollens sont d'inégale valeur biologique et que nous soupçonnons la nécessité pour la ruche de consommer des mélanges de pollen. I'origine de certaines anonialies dans le comportement des colonies, certains accilents, peut-être certaines maladies sont à reporter sur l'insuffisance ou sur les déficiences qualitatives d'une flore mal équilibrée et qui ne satisfait pas tous les besoins de la rucle. L'essaimage intempestif de certaines races d'abeilles n'est-il pas à reporter sur la nature de la flore locale, tout an moins en partie $I$.

I1 nous semble donc qu' une véritable "diétítique " de la muche reste à étudier en approfondissant d'une part la nature des besoins des abeilles et d'autre part la façon dont une flore domée peut les satisfaire en totalité. Le cycle biologique de la colonie d'abeilles en fonction de la flore et du climat reste un sujet d'étude qui mérite qu'on s'y consacre.

Le probleme de l'acilimatution que nous n'avons fait qu'eflleurer, reste à revoir en tenant compte des adaptations possibles à des types de fluraisons particuliers et à des régimes alinentaires variés. In fait de la dominance de certains types de végétation (landes, garrigues, forêts, cultures etc.) en un lien, les colonies présentent dess cycles biologiques adaptés ainsi que nous pensons l'avoir prouvé atr chapitre III. Les succès on les échecs que l'on enregistre lorsqu'on utilise des colonies d'abeilles en dehors de leur région d'origine sont à considérer sous l'angle des possibilités d'adaptation à un régine nouveau. I'apiculture transhumante, sonvent si décevante, aurait certainement le plus grand intérêt à connaitre less règles en matière d'acclimatation, règles qui restent d'ailleurs à définir. Il est probable que les perturbations apportées aux colonies par le changenent de milieu sont plus importantes que celles qui 
proviennent du déplacement lui-méme mais nous n'en connaissons pas encore la nature exacte.

On peut supposer, entre autres, que des ruches adaptées depuis toujours à une estivation caractérisée s'accommodent mal d'un rythne végétal faisant intervenir d'abondantes foraisons en été et un hivernage précoce. Tout reste à faire dans ce donaine; les données de la géographie botanique, de la phénologie, de la climatologie, de l'agronomic devront être confrontées aux données de la biologie de l'abeille ; un vaste domaine de recherches reste onvert de ce côté. 


\section{CONCILIONA GENERALES}

Nous arons envisagé le problème de la récolte du pollen par les abuilles sous deux aspects essenticls : comportement des butincuses, nutrition de la colonie.

\section{A. CARACTERES GÉNÉRAUX DU BUTINAGE}

( 11 ne peut valablement séparer la récolte du pollen des autres activités de récolte dont elle ne représente qu'un cas particulier. I'étude bibliographique du butinage en général nous a montré que la colonic d'abeilles récolte au sein du milieu extérieur les produits indispensables à sa nutrition et, dans une certaine mesure à ses activités constructrices (propolis). I les règles précises régissent 10 butinage ; on pent les énoncer ainsi :

Le butinage est l'aure collective de toute la ruche; chaque butineuse agit à l'extérieur en fonction de stimuli (1ui émanent de la colonie tout entière. La rícolte est lexpression des nécessités vitales, donc des besoins de la ruche. La récolte de l'eau fournit l'exemple le plus clair sans donte des liens qui existent entre le travail de collecte à l'extérieur et un besoin physiologique collectif : la soif. I a récolte de l'eau n'a lien que dans la mesure où, à l'intérieur de la colonie cles ouvrières ressentent le besoin physiologiune de diluer les sucres qu'elles consomment. Dès que le besoin est satisfait sur l'ensemble de la colonie l'activité des pourvoyeuses d'cau cesse totalement. Il en est de même pour le nectar qui n'est récolté que dans la mesure où le milieu interne s'en montre avide, ce qui est presque toujours 1e cas d'ailleurs. I'our le pollen le problime est plus complexe et nous ne connaissons qu'imparfaitement le mécanisme qui régit sá récolte : il tu est de mêne de la propolis. I a châne des stimuli partant de la ruche pour aboutir à l'individu récoltant du pollen ou de la propolis nous est incomplètennent connuc.

L hio arganisation sociale efficace qui se manifeste par les clanses dont $\therefore$ I'Rrscir a donné l'explication, permet par le jell constamment remanić de stimuli-signes, d'orienter la grosse masse des butineuses vers les soures de nourriture, pollen ou nectar, les plus rentables. La loi du moindre effort régit on effet le butinage : les sources de nectar concentré sont préférées aux sources de nectar dilué, les sources proches aux sources éloignées. C"est l'intensité des stimuli que constituent les clanses qui règle les déplacenents de populations de butincuses. Pour le pollen nous savons que les danses préscntent la même signification que pour le nectar. 
'Toutefois rien ne permet de comprendre comment se fait le choix des plantes pollinifères au sein de la flore locale. Lorsqu'on sait que le pollen constitue une denrée fortenent hétérogène par sa composition chimique et sa valeur nutritive on est amené à supposer que ces deux éléments peuvent jouer un róle essentiel dans la récolte.

Parmi les butinenses existe me ninorité infine d'individus que l'on peut qualifier de "particuliêrement donés" et dont la fonction est de clécouvrir constamment de nouvelles sources de nourriture. 'Toute abeille peut jouer à un moment de son existence de butineuse le rôle d'clarirense. I'élite des éclaireuses constitue l'antidote indispensable à l'autonuatisme avengle du butinage au sens habituel du terme. lille permet à toute colonie l'ajustage constant de la répartition des forees sur des étendues fleuries dont les fluctuations sont constantes.

Du point de one de la nutrition on sait que le pollen ionte wh rôle essentiel dans la vie des ruches dont c'est le seul aliment aroti. On sait que le pollen est indispensable à l'élevage des larves et qu'il constitue la nourriture des jeunes abeilles an moment oì elles deviennent nourrices. 'Tous les pollens n'ont pas la même action physiologique sur les glandes pharyngiennes, le corps adipenx et les ovaires. On discute encore toutefois sur la nature des éléments dı pollen jouant le rôle primordial dans leur valeur biologique. Pour les uns, seules les protéines comptent; pour d'autres des ćléments tels que les vitannines jouent un rôle non négligeable.

Nos propres recherches ont visé à étendre nos comnaissances dans le seul domaine de la récolte du pollen. Nous arons accumulé sur plusieurs années dess données d'ordre quantitatif et d'ordre qualitatif par la pesée, l'analyse pollinique et l'analyse chimique dess récoltes.

\section{3. - LA RÉCOLTE DU POLLEN CONSIDÉREE DU POINT DE VUE QUANTITATIF}

L'étude quantitative des récoltes nons a permis de rutronver les chiffres communément adoptés pour l'estimation des besoins d'une colonie normale pour tne année, soit 25 à $30 \mathrm{~kg}$. Cette quantité correspond à l'éclosion de 20000 abeilles environ.

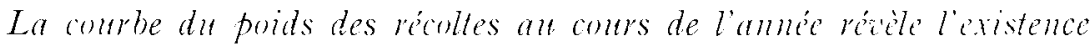
d'un cycle anmuel assea constant; un mininum absolu se place en hiver, un maximum en mai-juin, 111 minimum relatif en juillet-aont et une reinontée monentanée en septenbre. Ce cycle est caractéristique non seulement de la région parisienne mais également d'un grand nombre de régions de 1'Europe occidentale et contrale. Ines variations locales plus on moins inportantes peuvent se présinter avec décalage du maxi- 
mu1n et des minima en fonction de données floristiques et climatiques particulières. I,'Furope méridionale s'écarte sensiblement du schéma ci-dessus; les conditions locales tendent à inverser les deux minima : l'hivernage derient secondaire alors que l'estivation représente le point bas de la courbe. C'est pourquoi nos cssais sur l'acclimatation ont fait appel à des ruches du Mirli de la Iirance.

Facteurs influant sur la quantité de pollen récoltée. - Sujette à un constant remaniement de nature quantitative et qualitative la récolte du pollen est sous la dépendance de factenrs internes et de facteurs externes dont il est délicat de distinguer l'importance rative. On entend par factenrs internes le patrimoine héréditaire de la reine, la reine elle-même ct les produits de sa ponte, l'état physiologique de la colonie, ses caractères plus ou moins adajtatifs, lixés ou non génétiquenent, enfin tout son passé représenté par la population adulte, les réserves de nourriture, l'état d'utilisation des constructions, etc. I,es seuls facteurs externes ayant retenu notre attention sont la flore ct le climat.

La reine et le contain. - I e róle alimentaire du pollen laissait supposer une liaison directe entre l'étendue du comoain et le volume des récoltes. Il en est bien ainsi d'une façon générale. La reine ne constitue pas un. stimulus ialable par elle-même. İlle n'agit qu'à travers la ponte. J,es colonies les plus développées sont aussi celles qui récoltent le plus de pollen. Ainsi que l'a montré CHavvin (I950) la corrélation entre récolte du pollen et étendue du couvain n'est pas immédiate; ce n'est qu'à longue échéance qu'on trouve la liaison. Nous supposons, d'accord en ceci avec Chalvin (1956) qui s'appuie sur l'expéricnce, (1u'il doit exister chez la reine un rythme d'activité gúnésique complexe pouvant faire intervenir sinon des diapauses vraies du moins des repos saisonniers à caractère plus ou moins obligatoire. I.e rythme saisonnicr de ponte de la reine constituerait une base biologique accordée au climat et au rythme saison11ier des floraisons d'un lien. Chaque rucle présenterait donc une adaptation an cycle végétal de son lieu d'origine par l'intermédiaire du cycle de ponte de la reine. Il en résulterait un développenent harmonieux des colonies adaptées à leur nilieu et des perturbations plus ou moins profondes en cas de changenent brutal de l'environnenent.

Liorigine géographique des colonies. - Nous avons recherché s'il ctait possible de retrouver dans la courbe pondérale des récoltes de ruches d'origines diverses transplantées en un mêne endroit au cours de l'hivernage, les anomalies que laisse prévoir notre hypothèse. Effectivenent, la courbe des récoltes de pollen de mohes en provenance du Midi méditerranéen présente un décalage dans le temps vers une plus grande précocité. Dans le même temps l'étendue du couvain s'accroît plus rapidement que clans les ruches d'origine septentrionale. Nous retrouvons là un phé- 
nomène qui a été décrit chez de nombreux animaux ; Rana fusca capturée a Davos ( $1600 \mathrm{~m})$ et transportée en plaine pond l'année suivante à la date alpine (2-20 mai) et non à la date habituelle des Rana de la plaine. I.es exemples du même ordre sont nombreux (CUnNot I95I).

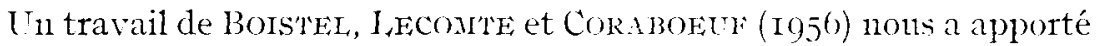
une autre confirmation de notre hypothèse. Fitndiant les courants d"action dans l'antenne isolée d'abeille, ils ont montré que les réponses aux stimuli olfactifs sont de 20 p. Ioo chez les abeilles d'hiver et de $90-95$ p. Ioo chez les abilles d'été. lin comparant au début de mars i955 les réponses fournies par nos ruches du groupe $\mathrm{P}$ (origine méditerranéenne) et par les ruches de la région parisienne ils ont obtenn to p. Ioo de réponses pour les abeilles du Midi contre 20 p. Ioo seulement pour les abeilles locales. Le taux de réponses étant en rapport avec l'état physiologique des abeilles, ils admettent que les ruches du Midi étaient dans un état physiologique plus proche de celui des abeilles d'été que les ruches du Nord.

Nons noterons encore que dans le Jura oì les neiges penvent parfois couvrir le sol pendant une partie du premier printemps on trouve sous la neige des colonies déjà bien développées en mars; en l'absence de toute récolte et malgré des conditions climatiques très dures, les ruches, vivant sur leurs réserves, se développent très bien. le cémarrage de la ponte de la reine n'est donc pas lié aux premières récoltes ni au réchauffement mais il se fait en fonction d'un rythme inné ou acquis nnais adapté aux conditions locales. Ise rôle joué par la durée du jour dans ce rỵthme est peu connu et mériterait une étude approfondie. On sait déji (RENxer I955) que l'abeille possède un rythme interne basé sur la période de 24 heures; il s'ajoute peut-itre à ce ruthme journalier un rythme annuel. dont la reine serait dépositaire.

Le butinage peut-il être assimilé an hoarding? La récolte du pollen se poursuit indéfininent jusque dans les colonies les phus désorganiséén mais clle atteint alors un niveau extremement bas. Il s'agit donc d'une activité essentielle du'il est pratiquement impossible d'inhiber. I, seul moven de ramener à zéro la récolte du pollenl, et ceci pour un tempss très court, c'est de suppriner les butineus's par un déplacement de la ruche. I,e tem1ss mis par lar colonie pour se réorganiser à la suite de cette anputation est de l'ordre de 3 à + jours.

Jes irrégularités constatées dans la quantité de pollen récoltéc nous ont amenés à reconnaître parmi les ruchess expérimentales de's types diffírents. Ia grande majorité des colonies présentent un comportement moven et récoltent entre 25 et $30 \mathrm{~kg}$ de pollen par an. Mais quelques colonies dépassent nettement ce's chifires et font des récoltés dépalssant $4^{\circ} \mathrm{kg}$, Ces colonies anormales sons le rapport de la quantiti de pollen récoltée sont des colonies très prolifique's et rolontiers essaimeuse's. Leurs besoins en pollen sont de ce fait supérieurs à la normale mais tout nous 
porte à croire que la notion de besoins dans la ruche mérite un examen attentif et qu'il ne s'agit pas d'une notion simple, parfaitement définie et applicable à toutes les ruches.

Si la colonie d'abeilles ne récoltait jamais sa nourriture qu'en fonction de ses besoins réels immédiats on lointains (réserves d'hivernage) et tels qu'un diététicien pourrait les définir sur des bases physiologiques, l'apiculture n'existerait pas. Il nous parait infiniment plus probable que la ruche n'a qu'une notion très imparfaite, peut-être nulle, des limites supérieures de ses besoins physiologiques et qu'clle amasse les provisions. tant que ses constructions lui permettent de les stocker. Iinsuite intervient généralement l'essaimage; en effet, le mécanisme de l'essaimage le plus couramment admis fait intervenir la congestion du nid à couvain, la saturation des besoins et le manque de place pour étendre les constructions. 'Tous les praticiens savent qu'on peut liniter souvent l'essaimage en fournissant en temps utile à la ruche un espace vide suffisant permettant d'entreposer l'excès de récolte et aussi en décongestionnant le nid à couvain. I, essaimage serait donc un processus analogue à une veproduction asexuée intervenant lorsque le milieu apporte en très grande abondance les éléments nutritifs et lorsque la taille de l'organisme qui se divise atteint une valeur critique.

Les prorisions de miel et de pollen des colonies sauvages sont souvent sans rapport avec les besoins réels, à tel point qu'elles finissent généralement par s'altérer d'une manière ou d'une autre. De nombreux exemples du même ordre peuvent être trouvés clans tout le règne animal ; les greniers de certaines fourmis moissonneuses par exemple, les réserves de nombreux rongeurs 11'ont pas de caractère d'utilité réelle et surtout ne sont pas proportionnés à des besoins déterminés. I)ans tous ces cas l'activité d'amassage dépasse son but et s'exerce pour elle-nnêne ainsi que le prouvent les études sur le hoarding chez le Rat blanc.

Si l'on considère que le hoarding a son origine dans une priation de nonrriture à un moment de l'existence du Rat, qu'il est influencé par des facteurs externes et qu'il s'agit d'une activité inégalement manifestée par les différents individus, il devient possible d'assimiler le butinage e'n géncral à the actirité de hoarding. I)ans la ruche la privation de nourriture est chronique; chaque période de manvais temps, chaque baisse du niveau des floraisons, chaque baisse des réserves est ressentie commuc une frustration de nourriture et déclenche, ainsi que nous l'avons montré, une activité de butinage plus considérable lorsque les conditions releviennent normales.

Facteurs externes. - Dans la période d'expansion, les besoins des colonies sont pratiquement sans linites mais les facteurs du milien, flore et climat, se chargent d'en fixer les bornes. Nans la région parisienne la flore est limitante an printemps et (n1 autonnne. I.es factenrs externes 
n'apparaissent que comme des freins entravant de façon discontinue une tendance expansive continue.

Facteurs particuliers. Modifications durables. - En fait, les tendances expansives illinitées sont inégalenent représentées chez les différentes colonies. Toutes les ruches n'ont pas les mèmes facultés d'assimilation et de mise en réserve des surplus alimentaires. Il existe donc, à côté des facteurs externes des facteurs internes d'ordre physiologique et sans doute aussi d'ordre génétique qui ont une valeur limitante. I,es possibilités de ponte de la reine sont plus ou moins grandes; les onirières sont douées d'une actirité et d'une aptitude au butinage relatirement rariable; on sait que, placées dans des conditions analogues de milieu, dotées de reines de valeur équivalente, des colonies roisines présentent des récoltes différentes. Iin somnne nous devons considérer chaque colonie connme tune entité, louée d'une certaine "personnalité" si l'on peut dire.

Von Firisch (I 947 ) observant des butineuses de pollen a constaté qu'elles sont donées d'une aptitude très variable pour la confection des pelotes ; certaines abeilles confectionnent une charge parfaite en un temps record; d'autres semblent perdre leur temps et ne réalisent (qu'un travail mérliocre.

Ce qui est vrai des butineuses prises individuellennent l'est aussi des ruches. Si nous considérons les spectres de dispersion des récoltes que nous avons mis en évidence il y a là l'indication très nette d'une méthode de travail fort différente chez les ruches concentrées sur wne récolte et chez les ruches dispersées. Il est vraisemblable que les ruches dispersées possèdent, pour une raison qui nous échappe encore, un nombre bien plus éleré d'éclareuses. Etant donné que nous trouvons la dispersion maximum chez les ruches les plus médioctes, les moins douées pour la rícolte, on est en droit de se demandere si la dispersion tris grande n'est pas la manifestation d'une situation matériclle critique pour la colonie. Se trouvant en état d'infériorité par suite de conditions internes défavorables la ruche réagit en cherchant dans toutes les directions possibles la solution de ses problèmes alinentaires. Toute source nourelle signalée par une éclaireuse prend une grande valeur significative et déplace un grand nombre de butineus:s. Mais cette supposition n'est pas forcément exacte; la dispersion n'est peut-ître que la manifestation d'aptitudes médiocres pour le butinage. Il parait difficile de trancher cette question.

I a constatation de la variabilité du " caractère" des colonies d'abeilles nous semble revêtir une grande importance théorique et pratique. On soupçonnait déjà l'existence d'un grand nounbre de races physiologiques (GONMARsk I953); il semble qu'il faille aller plus loin et qu'au-delà de caractères raciaux on doî̀e envisager l'existence de nombrenses modifications durables (Dauervariationen) portant sur le cycle biologique en général, sur l'aptitude an butinage (ou aptitude au hoarding) et sur les 
habitudes alimentaires. I'influence du milieu sur le comportement nous apparait donc comme fondamentale.

D'un point de vue strictement pratique une meilleure connaissance de ce qu'on nomme habituellement les "races locales " devrait permettre d'éviter les erreurs fréquemment commises par les apiculteurs qui se procurent des essaims dans des régions dont les caractères floristiques et climatiques sont par trop différents de ceux de la région où ils introduisent ces essaims. I es problèmes d'acceptation des reines, à court terme comme à long terme (phénomènes de supersédure) sont peut-être en relation avec des modifications durables présentes chez la reine et qui peuvent être incompatibles avec celles des ouvrières.

\section{C. - LA RÉCOLTE DU POLLEN CONSIDÉRÉE DU POINT DE VUE QUALITATIF}

Si l'on compare la nature botanique des récoltes d'un certain nombre de ruches placées au même endroit on constate une certaine variabilité. On ne retrouve pratiquement jamais deux ruches dont les récoltes soient semblables. Les plus grandes différences se présentent chez les plantes de moindre importance. L'accord se fait mieux sur les plantes essentielles fournissant les plus forts pourcentages de la récolte.

La fidélité aux sources de nourriture se manifeste par le fait qu' $u n$ pollen est récolté d'autant plus longtemps et d'autant plus intensément qu'il a été découvert plus tôt. Cette règle n'est pas absolue mais elle est vérifiée dans un grand nombre de cas.

De la variabilité des formules polliniques adoptées par les différentes ruches on peut conclure à l'existence du " goût " de certaines ruches pour des pollens particuliers. On trouve confirmation de cette hypothèse dans deux faits différents. Tout d'abord l'extension du goutt pour une Crucifère, le Colza, à plusieurs autres Crucifères ; une colonie ayant butiné en avril-mai sur les fleurs de Colza parait présenter une plus grande attirance pour les Moutardes qui fleurissent en juin-juillet que les colonies ayant peu visité le Colza; nous l'avons constaté sur les ruches mises en observation en 1950 .

Par ailleurs, si l'on compare les récoltes d'une année sur l'autre chez des ruches qui sont restées au même endroit et qui n'ont subi aucune modification importante, on constate une certaine continuité des gô̂ts. Cette continuité affecte exclusivement semble-t-il des plantes essentielles telles que le Saule (ex : ruches B en 1953 et I954) mais n'atteint guère les plantes secondaires. Il s'agit peut-être d'un chimiotropisme présenant des variations d'une colonie à l'autre.

Rôle de l'origine géographique. - Nous avons voulu voir si la variaAmales des Abeille's. - 19.59 . 
bilité des formules polliniques à l'intérieur d'un groupe de ruches aussi homogènes que possible n'est pas plus faible que la variabilité obtenue lorsqu'on compare entre eux des groupes de ruches d'origines géographiques différentes (Bouches-du-Rhône, Jura, Indre, Seine-et-Oise). Nons avons effectivement trouvé que la formule du butinage, ou mieux le spectre pollinique des récoltes est un caractère du groupe de ruches.Autrement dit l'origine géographique des colonies joue un rôle important dans l'utilisation qu'elles font de la flore d'un lieut. Il restait à savoir sur quelle base se fait le choix an sein de la flore.

Autres facteurs conditionnant le choix des abeilles. - Il est certain que le nombre des facteurs qui interviennent pour déterminer le choix des pollens récoltés est élevé. I Les plantes fournissant pollen et nectar à la fois sont souvent préférées aux autres. Les plantes communes existant en peuplements denses sont également préférées. La localisation et divers facteurs écologiques peuvent aussi jouer un rôle, nous n'en doutons pas, mais il ne nous a pas été possible de mettre en évidence nettement dans nos conditions expérimentales, l'existence d'une base purement écologique du butinage.

I1 ne semble pas par ailleurs que l'abeille recherche dans une flore nouvelle les éléments d'une flore anciennement connue; l'abeille ne connaît pas la botanique, si l'on peut s'exprimer ainsi. Si elle est adaptée à un certain rythme de floraison et à des caractères particuliers de la flore qu'elle exploite habituellement elle semble toutefois incapable de retrouver dans une flore nouvelle pour elle les quelques éléments dispersés de la flore ancienne. Il est bien entendu que nous faisons ici allusion à une mémoire collective et ancestrale dont le mécanisme est inconnu et non à la mémoire individuelle de chaque abeille basée sur l'expérience personnelle et qui est, elle, au contraire très fidèle.

Par contre, tout démontre clairement que l'abeille est capable de sélectionner les pollens en fonction d'un certain nombre de caractères qui sont liés à leur valeur alimentaire et à leur valeur biologique. Ceci concorde étroitement avec ce que nous savons du butinage du nectar; l'abeille reconnaît parfaitement la concentration en sucres d'un nectar et même sa composition chimique ainsi que l'a montré Wykes (I95I) qui a noté des préférences très nettes pour certains sucres. Il nous semble désormais certain que l'abeille est à même d'apprécier par une voie sensorielle $l a$ qualité d'un pollen et qu'elle fait son choix, au moins en grande partie, en fonction de cette qualité.

A la lumière de nos essais les plus récents sur les substances attractives $d u$ pollen, nous pensons que le choix des abeilles se fait en fonction d'une cascade de stimuli hiérarchisés. I, stimulus de base est la pulvérulence du produit; il reste efficace lorsque toutes les autres qualités ont 
disparu pour peu que l'abeille soit poussée par un besoin pressant de pollen (récolte des poudres inertes). Après la pulvérulence interviennent concurremment :

Io La teneur en substances attractives (chimiotropisme) qui font qu'une poudre qui les contient devient du pollen.

$2^{\circ}$ La valeur alimentaire.

Sous ce rapport, l'abeille ne se distingue done pas des autres animaux qui choisissent leur nourriture d'une façon précise à ceci près que dans le cas du pollen le choix est fait par une abeille alors que la consommation est le fait d'autres abeilles. Il n'est pas d'exemple d'animal consommant indifféremment n'importe quel aliment; tous les animaux exercent un choix et se montrent d'autant plus exigeants sur la qualité de la nourriture que les possibilités de la choisir sont plus grandes. L'abeille ne fait pas exception et l'existence d'un cycle annuel de la teneur en azote du pollen récolté concordant parfaitement avec l'importance des floraisons prouve nettement que plus la flore est variée et abondante (mois de mai dans notre région) plus la teneur en azote des récoltes est élevée. Le phénomène est général et intéresse toutes les ruches avec des variantes de faible amplitude sur lesquelles il convient de s'étendre quelque peu.

L'étude de ces variantes permet de constater que ce sont les ruches les plus précoces, les plus actives et par conséquent les plus développées qui recherchent les pollens les plus riches. Le qualitatif vient se superposer au quantitatif. Nous avons déjà vu que les ruches d'origine méditerranéenne ont tendance à récolter plus tôt de plus grosses quantités de pollen. Non seulement elles récoltent un pollen plus abondant mais aussi un pollen plus riche. Les ruches tardives, mal développées, mal douées pour la récolte ont une activité plus dispersée et récoltent des quantités plus faibles d'un pollen plus pauvre.

\section{D. - IMPORTANCE DE LA RÉCOLTE DU POLLEN DANS LE CYCLE BIOLOGIQUE DE LA RUCHE}

Nous interpréterons l'ensemble des faits que nous venons d'exposer en les situant dans le cadre général du cycle biologique annuel de la ruche. Nous pouvons nous représenter comme suit l'enchaînement des processus biologiques qui règlent la vie de la colonie : la reine représente un certain potentiel de ponte soumis à un rythme prédéterminé accordé au milieu habituel. Les abeilles permettent, ou ne permettent pas à la reine d'exprimer ses potentialités; elles la nourrissent bien ou mal ; elles acceptent sa ponte ou elles l'éliminent partiellement (CHauvin I956). De son côté la reine peut être ou ne pas être en synchronisme avec la colonie; elle 
peut être bonne ou mauvaise pondeuse sans qu'il en résulte la moindre différence dans les qualités intrinsèques des abeilles auxquelles elle donne le jour. Elle peut être bonne pondeuse mais se trouver dans une colonie mauvaise éleveuse qui élimine constamment une certaine proportion des cufs pondus. Il faut donc une bonne harmonie, un bon synchronisme entre la ponte, l'élevage des larves et la récolte de la nourriture pour que les besoins de la colonie soient maxima, donc que les récoltes soient élevées. Il suffit que la reine soit déficiente ou que les abeilles présentent une infériorité quelconque dans le domaine du butinage (faible aptitude à l'amassage) ou de l'élerage (mauvais métabolisme) pour que l'harmonie soit rompue.

Nous pouvons dire que nos ruches du Midi (groupe P) ont récolté très tôt un pollen abondant et riche en azote parce que leur reine était prolifique, dotée d'un rythme précoce de ponte et parce que les abeilles présentaient au plus haut point l'activité et les aptitudes nécessaires au butinage. I'impulsion donnée par la ponte, entretenue par des récoltes importantes d'une nourriture de bonne qualité conduit au développement optimum de la colonie. Nos ruches les plus médiocres (groupe R) ont présenté une lacune sous un rapport quelconque : reine défectueuse ou à rythme de ponte très tardif, faible aptitude au butinage des ouvrières ; l'un de ces facteurs suffit à expliquer à lui seul des récoltes plus faibles et un choix moins efficace des pollens.

I a ruche nous apparaît finalement, si nous voulons nous résumer, comme un organisme complexe soumis à l'action de facteurs internes (physiologiques, psychologiques, génétiques) et d'ordre externe (flore, climat). I.es régulations évoquées par Grassé (I950) interviennent constamment pour maintenir l'équilibre entre toutes ces actions. Aucun facteur ne peut être considéré comme étant à lui seul totalement déterminant d'un phénomène aussi complexe que le butinage.

\section{E. - LA FLORE UTILISÉE PAR LES ABEILLES}

Si l'on considère que sur toute l'étendue de l'Europe septentrionale l'abeille utilise sensiblement les mêmes plantes, on peut admettre qu'il existe une flore typique de l'abeille. Chaque biotope particulier (prairie, forêt, lande, etc.) offre à l'abeille un ensemble de plantes caractéristiques. I'analyse de récoltes d'origines très diverses montre une corrélation satisfaisante entre l'utilisation du sol et le tapis végétal d'une part et la nature des pollens récoltés d'autre part. On pourrait donc éventuellement considérer l'abeille comme un bon agent d'investigation dans le domaine de la géographie botanique. 
I a dominance locale de biotopes particuliers à flore restreinte permet d'envisager l'existence de conditions de vie très spéciales qui ont pu influencer profondément les caractères physiologiques des colonies vivant habituellement dans ces biotopes. L'étude des modifications apportées au cycle biologique et d'une façon générale au comportement des abeilles reste un domaine d'investigations largement ouvert aux recherches. 


\section{RÉSUMÉ}

I. I a récolte du pollen par les abeilles a été étudiée de I950 à I956 à la Station de Recherches apicoles de Bures-sur-Yvette, au moyen de la trappe à pollen, instrument permettant la capture de Io p. Ioo environ des pelotes de pollen rapportées à la ruche. Le but du travail était de préciser dans quelles conditions se fait cette récolte, tant du point de vue quantitatif que du point de rue qualitatif, et de mettre en évidence l'origine des variations couramment observées.

2. I a quantité moyenne de pollen récoltée par ruche et par an paraît osciller entre 25 et $30 \mathrm{~kg}$. Ceci est en accord avec les calculs que l'on peut faire par ailleurs et avec les données bibliographiques.

3. I e maximum de récolte journalière se situe habituellement aux environs de I $\mathrm{kg}$ ( IoO g à la trappe) et atteint exceptionnellement $2 \mathrm{~kg}$ pour certaines colonies très populeuses.

4. I'allure de la courbe annuelle de récolte est relativement constante en un lieu donné. I_es maxima et mininı́ se situent toujours approximativement aux mêmes époques.

5. I'absence de la reine ne constitue pas, en soi, un stimulus suffisant pour inhiber la técolte qui se poursuit dans les colonies les plus désorganisées à un niveau, il est vrai, extrêmement bas.

6. I a quantité de pollen récoltée est pratiquement proportionnelle à 1'étendue du couvain. Toutefois quelques colonies semblent présenter une propension très nette à récolter du pollen au-delà de leurs besoins normaux.

7. On ne constate ancune corrélation nette entre récolte du pollen et récolte du nectar. Il s'agit de deux processus distincts.

8. I a comparaison entre la récolte du pollen et un phénomène très général bien étudié chez le Rat blanc, l'amassage ou hoarding, a permis de mettre en évidence certains points communs. Il semble que le butinage en général soit assimilable en grande partie à une activité de hoarding. Les deux phénomènes sont influencés par le milieu et en particulier par la température. Ils sont sous la dépendance d'une privation de nourriture, laquelle est chronique dans la ruche. Tous deux aboutissent à la constitution de réserves alimentaires qui sont souvent sans commune mesure avec les besoins physiologiques stricts. I'homme a pu, par la sélection, contribuer à développer l'instinct de hoarding chez les abeilles.

9. L'instinct d'amassage porte à la fois sur le nectar et sur le pollen. Lorsqu'il porte sur le nectar il aboutit à la constitution de réserves sucrées. Lorsqu'il porte sur le pollen il marque en général une tendance à l'expansion indéfinie du couvain et à l'essaimage.

ro. Selon l'origine géographique des colonies la courbe de récolte 
du pollen prend une allure un peu différente. Eille marque une plus grande précocité chez les ruches d'origine méditerranéenne, ce qui est le signe d'un rythme de développement plus rapide.

II. La flore, considérée comme facteur agissant sur la récolte du pollen, n'apparaît comme un facteur limitant qu'en dehors de la période printanière.

I2. De tous les facteurs météorologiques étudiés, la température semble le plus important. I e rôle des facteurs météorologiques est purement limitant et s'exerce principalement au début du printemps et en automne. Aucune récolte n'a lieu au-dessous de $10^{\circ}$. Il n'y a pas proportionnalité entre la température et la quantité de pollen récoltée, sauf sur de très courtes périodes.

I3. Seule une partie de la flore d'un lieu est utilisée par les abeilles.

I4. Du point de rue qualitatif on constate de fortes variations dans les récoltes de ruches voisines d'origine non contrôlée. Chaque colonie fournit un spectre pollinique de récoltes qui lui est propre. Des "goûts " particuliers se manifestent chez certaines ruches pour certaines plantes.

I5. Le plus souvent, ane source de pollen est d'autant plus intensément utilisée qu'elle a été découverte plus tôt.

I6. Ise goût de certaines ruches pour un pollen déterminé peut intéresser non set1lement l'espèce, nais aussi la famille.

I7. Le facteur " découverte " des sources de pollen parait important.

I8. Des ruches homogènes par l'origine de la reine présentent encore des différences importantes dans la formule pollinique adoptée.

I9. D'une année sur l'autre les gôtts manifestés par une ruche pour certains pollens peuvent se retrouver; toutefois le phénomène ne semble intéresser que les plantes les plus importantes.

20. Si l'on étudie le spectre pollinique des récoltes de plusieurs groupes de ruches d'origines géographiques différentes, on constate que le spectre varie moins à l'intérieur d'un groupe que d'un groupe à l'autre.

2I. Certaines ruches concentrent leur activité sur un petit nombre d'espèces ; d'autres, au contraire, se dispersent sur de nombreuses plantes.

22. La recherche d'une base de comparaison entre les récoltes de groupes de ruches d'origines différentes permet d'exclure à peu près totalement des facteurs tels que la rémanence d'un comportement antérieur, ainsi que les facteurs écologiques et spatiaux.

23. La teneur en azote des récoltes de pollen subit un cycle annuel présentant un maximum en mai-juin.

24. La teneur en azote des pollens semble jouer un rôle essentiel dans le choix des butineuses; les différences qualitatives dans les récoltes des différentes ruches peuvent s'expliquer par la recherche dans le pollen de certaines qualités nutritives. 
25. Le pollen constitue pour l'abeille un produit défini par un certain nombre de qualités dont les principales sont la pulvérulence, la valeur alimentaire et la valeur biologique, la teneur en substances attractives.

26. I.es substances attractives du pollen appartiennent vraisem!blablement au groupe des stérols ou à un groupe chimiquement voisin.

27. I,es plantes dont l'abeille récolte le pollen sont sensiblement les mêmes dans toute 1'Furope occidentale.

28. Ie tapis végétal présente de grandes variations dans sa valeur pour l'abeille. I a dominance d'un biotope végétal en un lieu impose aux abeilles une adaptation particulière. On discute les rapports entre flore et cycle biologique des ruches.

29. Une étude détaillée des récoltes de pollen en divers points de la Firance permet d'établir la valeur pollinique des différents aspects du tapis végétal.

30. I'ensemble des travaux exposés permet d'envisager sous un jour nouveau les problèmes de la nutrition de la ruche et de l'acclimatation des abeilles.

\section{ZUSAMMENFASSUNG}

$I^{\circ}$ I Die Pollenernte der Bienen ist von $\mathrm{I} 950$ bis $\mathrm{I} 956$ am Institut für Bienenforschung in Bures-sur-Yvette mit Hilfe einer Pollenfalle studiert worden. Dieses Instrument ermöglicht es, ungefähr Io \% der zum Stock gebrachten Pollenhöschen abzunehmen. Die Arbeit setzte sich zum Ziel, die Bedingungen zu präzisieren, unter denen die Ernte vor sich geht; dies sowohl vom quantitativen, als auch vom qualitativen Standpunkte aus, und die Ursachen der häufig beobachteten Variationen zu ermitteln.

$2^{\circ}$ Die mittlere Pollenmenge, die in einem Stock während eines Jahres geerntet wird, scheint zwischen 25 und $30 \mathrm{~kg}$ zu schwanken. Dies stimmt mit den Angaben in der Literatur und mit anderseits gemachten Berechnungen iiberein.

$3^{\circ}$ Die maximale Tagesernte beträgt gewöhnlicherweise ungefähr I $\mathrm{kg}$ ( oo $\mathrm{g}$ in der Falle), und erreicht ausnahmsweise bei sehr starken Völker $2 \mathrm{~kg}$.

$4^{\circ}$ Der Verlauf der Jahreskurve für die Ernte ist für einen gegebenen Ort relativ konstant. Iie maxima und minima ergeben sich immer ungefähr zu den gleichen Zeitpunkten.

$5^{\circ}$ Das Fehlen einer Königin ist für sich allein kein genïgender Stimulus, um die Pollenernte zu verhindern, die auch in den am stärksten desorganisierten Kolonien weitergeht, allerdings in sehr geringem Masse.

$6^{\circ}$ Die gesammelte Pollenmenge ist praktisch ungefähr der Bedeutung der Brut proportional. Einige Völker scheinen immerhin eine deutliche Tendenz aufzuweisen, iiber ihre normalen Bedïrfnisse hinaus Pollen zu sammeln.

$7^{\circ}$ Man stellt keine deutliche Korrelation zwischen Pollen und Nektarernte fest. Es handelt sich um zwei getrennte Prozesse.

$8^{\circ}$ Der Vergleich zwischen der Pollenernte und der sehr generellen Erscheinung des Zusammenraffens, oder " hoarding ", die bei der weissen Ratte gut 
studiert wurde, ergibt gewisse gemeinsame Punkte. Es scheint, dass im allgemeinen das Einsammeln der Bienen mit der "hoarding " - . Tätigkeit verglichen werden kann. Beide Eirscheinungen werden vom Milieu, und insbesondere von der Temperatur beeinflusst. Sie hängen ab von einem Nahrungsmangel, der im Bienenstock chronisch ist. Beide führen zur Anlegung von Nahrungsteserven, die oft in keiner Beziehung stehen zu den strikten physiologischen Bediirfnissen. Der Mensch hat durch Selektion dazu beigetragen, den " hoarding " - Instinkt bei den Bienen zu fördern.

$9^{\circ}$ Der Zusammenraffungsinstinkt betrifft sowohl den Nektar, als auch den Pollen. Wenn er den Nektar betrifft, führt er zur Häufung von zuckerhaltigen Reserven. Betrifft er den Pollen, dann markiert er im allgemeinen eine 'Tendenz zur unbeschränkten Ausdehnung der Brut und zum Schwärmen.

IO $^{\circ} \mathrm{Je}$ nach der geographischen Herkunft der Kolonien hat die Pollen sammelkurve eine etwas verschiedene Beschaffenheit. Sie steigt früher an bei Stämmen aus dem Mittelmeergebiet, was ein Zeichen für eine raschere Entwicklung ist.

I I ${ }^{0}$ Die Flora, als die Pollen ernte beeinflussender Faktor, erweist sich nur ausserhalb des Friihjahrs als beschränkender Faktor.

I $2^{\circ}$ Von allen untersuchten meteorologischen Faktoren scheint die Temperatur der wichtigste zu sein. Die Rolle der meteorologischen Faktoren ist ausschliesslich eine beschränkende and wirkt sich hauptsächlich zu Beginn des Friihjahrs und im Herbst aus. Unterhalb Io findet keine Sammeltätigkeit mehr statt. Die Temperatur und die gesammelte Pollenmenge sind nicht proportional, ausser für ganz kurze Perioden.

$13^{\circ}$ Es wird von den Bienen nur ein Teil der Filora eines gegebenen Ortes verwendet.

I $4^{\circ}$ Bei Nachbarstöcken, deren Herkunft nicht kontrolliert wurde, stellt man vom qualitativen Standpunkt grosse Unterschiede in der Ernte fest. Jede Kolonie zeigt in der Ernte ein ihr eigentiimliches Pollenspektrum. Bei bestimmten Stöcken zeigt sich eine Vorliebe für bestimmte Pflanzen.

I $5^{\circ}$ Meistens wird eine Pollenquelle umso ausgiebiger verwendet, je früher sie entdeckt wurde.

I6 $6^{\circ}$ Die Vorliebe einzelner Stöcke für einen bestimmten Pollen kann nicht nur die Art, sondern auch die Familie betreffen.

I $7^{\circ}$ Die (Entdeckung) der Pollenquellen scheint ein wichtiger Faktor zu sein.

I $8^{\circ}$ Stöcke, die in bezug auf die Herkunft der Königin homogen sind, zeigen noch grosse Unterschiede in der adoptierten Pollenformel.

I $9^{\circ}$ Die Vorliebe eines Stockes für bestimmte Pollensorten kann von einem Jahr zum nächsten Jahr wiedergefunden werden, betrifft aber nur die wichtigsten Pflanzen.

$20^{\circ}$ Wenn man das Spektrum der Pollenernte mehrerer Gruppen von Stöcken von verschiedener geographischer Herkunft studiert, stellt man fest, dass das Spektrum stärker variiert zwischen den Gruppen, als innerhalb einer Gruppe.

$2 I^{0}$ Einzelne Bienenstöcke konzentrieren ihre Tätigkeit auf eine kleine Zahl von Arten, andere verteilen sie auf viele Pflanzen.

$22^{\circ}$ Der Vergleich der Ernte von Gruppen von Völkern verschiedener Herkunft gestattet es, Faktoren wie z. B. : Reminiszenzen friiherer Verhaltensweisen, oder ökologische und räumliche Faktoren auszuschliessen.

$23^{\circ}$ Der Stickstoffgehalt der Pollenernten unterliegt einem Jahreszyklus, der während der Monate Mai und Juni ein Maximum zeigt. 
$24^{\circ}$ Der Stickstoffgehalt des Blitenstaubes scheint bei der Wahl der Sammlerinnen eine wesentliche Rolle zu spielen. Die qualitativen Unterschiede in den Eirnten verschiedener Stöcke können durch die Suche nach Pollen eines bestimmten Nährwertes erklärt werden.

$25^{\circ}$ Der Pollen stellt für die Biene ein Produkt dar, das durch eine gewisse Zahl von Eigenschaften definiert ist, von denen die wichtigsten die Staubkonsistenz, der Nährwert und der biologische Wert, sowie der Gehalt an anziehenden Substanzen sind.

$26^{\circ}$ Die attraktiven Substanzen des Pollens dürften der Gruppe der Sterole angehören.

$27^{\circ}$ Die Pflanzen, auf denen die Biene Bliitenstaub einsammelt, sind in ganz Westeuropa ungefähr dieselben.

$28^{\circ}$ Der Pflanzenteppich zeigt grosse Wertunterschiede für die Biene. Das Vorherrschen eines Pflanzenbiotopes an einem Ort zwingt die Bienen spezifische Anpassungen auf. Es werden die Beziehungen zwischen der Flora und biologischem Zyklus der Stöcke diskutiert.

$29^{\circ}$ Ein genaues Studium der Pollenernten in verschiedenen Lokalitäten Frankreichs ermöglicht es, den Pollenwert der verschiedenen Aspekte des Pflanzenteppichs aufzustellen.

$30^{\circ}$ Die Gesamtheit der vorgelegten Untersuchungen gestattet es, die Ernährung des Bienenstockes und die Akklimatisation der Bienen unter neuen Gesichtspunkten zu betrachten.

\section{RÉFÉRENCES BIBLIOGRAPHIQUES}

Alfonsus (E. C.). - Pollenverbrauch von Bienenvölkern. Arch. $f . B k d ., 14$, (5), 220-3, I933.

Anderson (R. J.) et Kui,P (W. L.). -- Analysis and composition of corn pollen. J. biol. chem., 50, 433-453, I922.

Andrejew. - Die Pollen der Pflanzen, welche die Bienen sammeln. Arch. f. $B k d ., \mathbf{9},(8), 77$, I 928 .

Aucrair (J. L.) et Jamieson (C. A.). - Qualitative analysis of amino-acids in pollen collected by bees. Science, 108, (2805), 357-8, I948.

BELING (I.). - Beobachtingen über das Pollensammeln der Honigbiene. Arch. f. Bkd., 12, (7-8), 76-83, I93I.

Betrs (A. D.). - The constancy of the pollen collecting bee. The Bee world., oct. I935, III-II3, I920; juin-septembre I920, IO-II, I935.

BEUTT,ER (R.). - Biologisch-chemische Untersuchungen am Nektar von Immenblumen. $Z$. vergl. Physiol., 12, (I), 72-I76, r930.

Beutiler (R.). - Ueber die Flugweite der Bienen. Z. vergl. Physiol., 36, 266298, I954.

Bindra (D.). - Water-hoarding in Rats. J. comp. and Physiol. Psychol., 40, I $49-156$, I947.

Boch (R.). - Die Tänze der Bienen bei nahen und fernen Trachtquellen. Z. vergl. Physiol., 38, I36-167, I956.

Boistel, (J.), LeComte (J.) et Corabeuf. - Quelques aspects de l'étude électrophysiologique des récepteurs sensoriels des antennes d'Hyménoptères. Ins. Soc., 3, (I), 25-3I, I956.

Bonnier (G.). - Sur la division du travail chez les abeilles. C. R. Acad. Sc., 143, 94I-6, I906.

BötTchER (F. K.). - I/3 Zentner Pollen geerntet. Mein neues Pollenerntegerät. Leipziger Bienen-Ztg., 56, (2), x94I. 
BöTtcher (F. K.). - Mein Pollengerät und seine Bedeutung für die Bienenzucht. D. Imkerführer., 44, I943.

Buchner (R.). - Ueber das Verhalten zweier Bienenrassen beim sammeln. Z. f. Bienenforsch., 2, (7), 20I-208, I954.

Butle (C. G.). - The behaviour of bees when foraging. J. of Roy. Soc. Arts., XCIII, (n $\left.{ }^{0} 4699\right)$, 50I-5II, I945.

BUTLER (C. G.). - The method and importance of the recognition by a colony of homeybees (Apis mellifica $\mathrm{L}$ ) of the presence of its queen. Trans. $R$. ent. Soc. Lond., 105, (2), II-29, I954.

BUtler (C. G.), JefFree (E. P.) et Kalmus (H.). - The behaviour of a population of honeybees on a natural and on an artificial crop. $J$. of exp. Biol., 20, 65-73, I943.

Buzzard. - De l'organisation du travail chez les abeilles. Bull. Soc. Apic. des A. M., 15, (I 6 ), 65-70, 1936.

Van CAMPo-Duplan. - Considérations générales sur les caractères des pollens et des spores et sur leur diagnose. Bull. Soc. Bota. Fr., 101, (5-6), 250$28 \mathrm{I}, \mathrm{I} 954$.

CASTEEL (D. B.). - The behavior of the honeybee in pollen collecting. $U$. S. Dep. of Agric. Bur. Entom., Bull. I2I, 36 p., IgI 2.

Chauvin (R.). - Méthode d'observation continue de la ponte de la reine chez les abeilles. L'A piculteur (Sec. Sc.), 94, (II), I2 p., I950.

Chauvin (R.). - Aspects sociaux des grandes fonctions chez 1'abeille. La théorie du super-organisme. Ins. Soc., 1, (2), I23-9, I954.

Chauvin (R.). - Les facteurs qui gouvernent la ponte chez la reine des abeilles. Ins. Soc., 3, (4), 499-504, I956.

Chauvin (R.), Defromont (C.), Louveaux (J.) et Verge (J.). - Sur une substance présente dans le pollen qui s'oppose au développement de certaines bactéries. C. R. Soc. Biol., CXLVI, 645, I952.

Chauvin (R.) et Lavie (P.). - Recherches sur la substance antibiotique du pollen. Ann. Inst. Pasteur, 90, 523-7, 1956.

Chauvin (R.) et Louveaux (J.). - Les caractères de la récolte de pollen en I955. La flore française productrice de pollen pour les abeilles. L'A piculteur (Sec. Sc.), 100, (4), I2 p., I956.

COMíté NATIONAL DE GÉographie. - Atlas de France. Carte du tapis végétal. C. N. G. I2I, Bd Saint-Germain. Paris, I956.

Cuenot (L.) et TeTry (A.). - L'évolution biologique. Masson éd., Paris, I $95 \mathrm{I}, 588 \mathrm{p}$.

ECKER $(\mathrm{J}$.$) . - The flight range of the Honeybee. J. agric. Research, 4\%,$ (5), $257-285$, I 933 .

EAckerT (J.). - Pollen requirement by a colony of Honeybees. $J$. of econ. Ent., 35, (3), 309-II, I942.

ELSER (E.) et GANZMULiER (J.). - Die chemische Zusammensetz11ng einiger Blütenstaubarten. $Z$. physich. Chem., 194, 2 I, 1930.

Emerson (A. E.). - The superorganismic aspect of society. Colloque intern., C. N. R. S., 34, 333-353, Paris, I952.

Erdtman (G.). - Pollen morphology and Plant Taxonomy. Angiosperms. Stockholm, I952, $539 \mathrm{p}$.

Von Euler (H.), Heller (L.) et HogbERG (K.). - Nukleoproteide aus Pollen von Birke. Arkiv. Kemi Mineral. Geol., 26 A, (2I), I5 p., I948.

FAEGri (K.) et IVERSEN (J.). - Text book of modern pollen analysis. Ejnar Minskgaard. Copenhague, I950, I68 p.

Farrar (C. L.). - Bees must have pollen. Gleanings in Beeculture, 62, (5), I76-8, I934.

FARRAR (C. L.). - Influence of pollen reserves on the surviving populations of over-vintered colonies. Amer. Bee Jour., 76, (9), 452-5, I956.

Von FRISCH (K.). - Chr. Sprengels Blumentheorie vor I50 Jahren und heute. Naturwiss., 19, 20, 223-229, I943. 
Von FrIsch (K.). - Duftgelenkte Bienen im Dienste der Landwirtschaft und Imkerei. Springer Verlag. Vienne, I947, I89 p.

Von Frisch (K.). - The dances of the honeybee. Bull. anim. Behar., 1, I, I947.

Von FrISCH (K.). - Orientierungsvermögen und Sprache der Bienen. Naturwiss., 38, (5), I05-II2, I95I.

Gaussen (H.). - Carte de la Végétation de la France. Perpignan; I/200.000 ${ }^{\mathrm{e}}$. Publiée par le C. N. R. S. avec le concours du Ministère de l'Agriculture, I946.

Gendot (G.). - Eau nécessaire aux abeilles. L'apiculteur, 51, I64-8, I907.

Gontarski (H.). - - Uber physiologische Unterschiede bei Bienen verschiedener Abstammung. $Z$. $t$. Bienenforsch., 2, (3/5), 98, I08, I953.

Gontarski (H.). - Zur Brutbiologie der Honigbiene. Z. \%. Bienenforsch., 2, (I), 7-IO, I953 b.

Gontakski (H.). - Untersuchungen iiber die Verwertung von Pollen und Hefe zur Brutpflege der Honigbiene. Z. f. Bienenforsch., 2, (6), I6I-I79, I 954 .

Grassé (P. P.). - La régulation sociale chez les Isoptères et les Hyménoptères. Coll. intern. C. N. R. S., Paris, I952, XXXIV, 323-33I, I950.

De Groot (A. P.). - Effect of a protein containing diet on the longevity of caged bees. Koning. Nederl. Akad. Weten., 54, 2-4, I95I.

De Groot (A. P.). - Amino-acid requirements for groth of the Honeybee. Experientia, 8, (5), I92, I952.

De Groot (A. P.). - Protein and amino-acid requirements of the honeybee Physiologia comparata et cecologia, 3, (2/3) (90 p. (Apis mellifica L). Thèse), I $97-285$, I953.

Hammer (O.). - Investigations on the nectar-flow of red clover. Oikos, 1, (I), 34-47, I949.

Hare (Q. A.) et VANSELl (G.). - Pollen collection by honeybees in the Delta, Utah, Alfalfa seed producing area. J. of the Amer. Soc. of Agron., 38, (6), 462-9, 1946 .

Harris (W. F.) et Filmer (D. W.). - Pollen in honey and bee loads. N. $Z . J$. Sci. Tech. A., 30, (3), I78-I87, I948.

HAYDAK (M. H.). - Brood rearing by honeybees confined to a pure carbohydrate diet. $J$. of econ. Ent., 28, 657, 1935 .

HAYDAK (M. H.). - Comparative value of pollen and pollen substitutes. II. Bee Bread ans Soy bean flour. J. of econ. Ent., 33, (2), 397, I940.

HAYDAK (M. H.). - Larval food and development of castes in the Honeybee. J. of econ. Ent., 36, (5), 778-792, I943.

HAYDAK (M. H.). - The food of the Honeybee. In: Paddock F. B. Report of Iore'a State Apiarist for 1943, 68-72, 1944.

Haydak (M. H.). - Value of pollen substitutes for brood rearing of Honeybees. J. of econ. Ent., 38, (4), 484-7, I945.

HAYDAK (M. H.). - Causes of deficiency of soya-hean flour as a pollen substitute for Honeybees. J. of econ. Ent., 42, (4), 573-579, I949.

HAYdak (M. H.) et PALMER (L. S.). - Vitamin content of bee foods. II. Vitamin BI content of royal jelly and beebread. $J$. of econ. Ent., 33, (2), 396-7, I940.

HAYdAK (M. H.) et PALMer (L. S.). — Vitamin content of bee-Foods: III : Vitamin $A$ and Riboflavin content of bee bread. J. of Econ. Ent., 34, (I), 37-8, I94 r.

HAYdak (M. H.) et PALmer (L. S.). - Royal jelly and bee-bread as sources of Vitamine $\mathrm{BI}, \mathrm{B} 2, \mathrm{~B} 6, \mathrm{C}$ and nicotinic and pantothenic acids. $J$. of econ. Ent., 35, (3), 319, I942.

HIRSCHFELDER (H.). - Quantitative Untersuchungen zum Polleneintragen der Bienenvölker. Z. f. Bienenforsch., 1, 54, 67-77, I95I.

HrTchcock (J. I).). - A milk-digesting enzyme in pollen stored by honeybees. Amer. Bee J., 96, (I2), 487-9, I956. 
Hodges (D.). - The pollen loads of the honeybee. B. R. A. Ltd. London I952, 5 I pages.

HodGEs (D.). - The progressive packing of the pollen loads as revealed by colour patterns in mixed-loads. Bee World, 35, (2), 3I-32, I954.

HUNT (J. Mc V.). - The effect of infant Feeding frustration upon adult hoarding in the albino Rat. J. Abnorm. and Soc. Psychol., 36, 338-36o, I94I.

JAXTHEIMER (R.). - Die Ausnutzung der heimischen Flora durch die Bienen. Arch. f. Bkd, 26, I7-29, I949.

Kitzes (G.), Schue'tTe (H. A.) et Elvehjem (C. A. C.). - The B vitamins in Honey. J. Nutrition, 26, 24I, I943.

KLATZMans (J.). - La localisation des cultures et des productions animales en France. Imprimerie nationale. Paris, I955, $477 \mathrm{p}$.

I. ECOMTE (J.). - Sur le vol des abeilles butineuses. $Z, f$. Tierpsychologie, 13, (I), 26-30, 1956 .

Levin (M. D.) et BoharT (G. E.). - Selection of pollens by Honey bees. Amer. Bee J., 95, (Io), 392-3, 1955.

LEX ('T.). - Duftmale an Bliiten. $Z$. $f$. vergl. Physiol., 36, 212-234, I954.

LINDAUER (M.). - Uber die Einwirkung von Inuft und Geschmack stoffen sowie anderer Faktoren auf die 'Tänze der Bienen. $Z$. $f$. vergl. Physiol., 31, 348-4I2, I 948 .

LINDAUER (M.). - Ein Beitrag zur Frage der Arbeitsteilung im Bienenstaat. Z. f. vergl. Physiol., 34, 299-345, 1952 .

LINDAUER (M.). - Temperaturregulierung und Wasserhaushalt im Bienenstaat. Z. f. vergl. Physiol., 36, 39I-432, 1954.

LiNdauer (M.). - Schwarmbienen auf Wohnungssuche. $Z$. t. vergl. Physiol., 37, 263-324, I955.

LouveAUX (J.). - Observations sur le déterminisme de la récolte du pollen par les colonies d'abeilles (Apis mellifica). C. R. Acad.Sc., 231, 92 I-2, I950.

Louveaux (J.). - Études sur la récolte du pollen par les abeilles. L'A piculteur (Sec. Sc.), 98, (12), 8 p., I954.

Louveaux (J.). - Introduction à l'étude de la récolte du pollen par les abeilles. Physiologia comparata et acol., 4, (I), I-54, I955.

Louveaux (J.). - Remarques sur les facteurs conditionnant le choix par les abeilles (Apis mellifica) des plantes leur fournissant du pollen. C. R. Acad. Sc., 242, 2994-6, I956.

Lubliner-Mianowska (K.). - Les pigments du pollen (en polonais). Acta soc. bot. polon., XXIV, (3), 609-6I8, I955.

LUBLiner-Mianowska (K.). - Recherches sur la composition chimique du pollen. I) Étude du pollen comme nourriture du couvain. (En polonais). Acta Soc. Bot. Polon., XXV, (3), 409-4I9, 1956.

Lunden (R.). - Literature on pollen chemistry. Grana palynologica, 1, (2), 3-I9, I956.

MCGREGOR (S. E.). - Collection and utilization of propolis and pollen by caged honey-bee colonies. Amer. Bee J., 92, (I), 20-2 I, I952.

Maurizio (A.). - Die Hahnenfussvergiftung (Bettlacher Maikrankheit) im Frïhjahr r944. Schreiz. Bienen $Z$., 6, I944.

Maurizio (A.). - Trachtkrankheiten der Bienen. Vergiftungen bei einseitiger 'Tracht von Rosskastanien. Schweiz. Bienen Z., 1, (8), 337-368, I945 (Beihefte).

Maurizio (A.). - Beobachtungen iiber die Lebensdauer und der Futterverbrauch gefangen gehaltener Bienen. Schweiz. Bienen Z., 2, (I3), $4^{8}$ p., (Beihefte), I 946.

Máurizio (A.). -- Prüfung von Pollenersatzmitteln. Schweiz. Bienen Z., 74, (3), III-II8, I95I.

Maurizio (A.). - Weitere Untersuchungen an Pollenhöschen. Schweiz. Bienen $Z$., (Beiheft), 20, I953. 
MaUrizio (A.). -- Pollenernährung und Lebensvorgänge bei der Honigbiene (Apis mellifica). Ia andwirtsch. Jahrbuch der Scheeeiz., 68, (2), II5-I82, I954.

Maurizio (A.) et Kolmmañ (H.). - Pollenanalytische Untersuchungen an Honig und Pollenhöschen. C. Beobachtungen an Pollenhöschen. Schieiz. Bienen $Z$. (Beihefte), 2, (I8), 442-455, I949.

MEYER (W.). - Die "Kittharzbienen" und ihre Tätigkeit. $Z$. $f$. Bienenforsch., 2, (7), I85-200, I954.

MinderhoUd (A.). - Untersuchungen iiber das Betragen der Honigbiene als Bliitenstauberin. Gartenbauwissenschaft, 4, (4), 342-362, I03I.

Morgan (C.). - The hoarding instinct. Psychol. Rev., 54, 335-34I, I947.

MORGaN (C.), STELIAR (E.) et Johnson (O.). - Food deprivation and hoarding in Rats. J. comp. Psychol., 35, 275-295, r(943.

Munx (N. L.). - Handbook of psychological research on the Rat. Houghton Mifflin C e, Boston, ro50, $598 \mathrm{p}$.

Nelson (F. C.). - Adaptability of young bees under adverse conditions. Amer. Bee J., 67, 242-3, I927.

Niki.SEN (N.), Grommer (J.) et LUNDEN (R.). - Chemical composition of pollen from some plants. Acta Chem. Scand., 9, IIoo-6, I955.

$\mathrm{Zu}$ Oe'Ttingen-Spieiberg ('T.). - Uber das Wesen der Suchbienen. $Z . f$. vergl. Physiol., 31, 454-489, 1949.

Opringer (E.). - Zur Psychologie der Duftchressuren bei Bienen. $Z$. $f$. vergl. Physiol., 31, 44I-453, I949.

PAIN (J.). - L'alimentation et le développement des ovaires chez l'ouvrière d'abeille (Apis mellifica). Arch. int. P'hysiol., IIX, (2), 203-210, I95I.

Pain (J.). - Sur 1'ectohormone des reines d'abeilles. Ins. Soc., 3, (I), I99-202, I956.

PARK (O. W.). - Time factors in relation to the acquisition of food by the honeybee. Iora Agric. Exp. Station Res. Bull., I08, I928.

PARK (O. W.). - Activities of honeybees, in "Grout. 'The hive and the Honey-

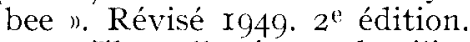

PARKer. - The collection and utilization of pollen by the honeybee. Connell Univ., 98 p., 1926.

Pearson (P. B.). - Pantothenic acid content of pollen. Proc. Soc. Exptl. Biol. Med., 51, 29I, I942.

Percival, (M.). - Pollen collection by Apis mellifica. The New Phyt., 46, (I), I $42-173$.

Percival (M.). - Pollen presentation and pollen collection. The nere Phyt., 49, (I), 40, I950.

Percival (M.). - The presentation of pollen in certain Angiosperms and its collection by Apis mellifica. The new Phytol., 54, (3), 353-368, I955.

RErTER. - The coloration of anther and corbicular pollen. The Ohio J. of Sc., XIVII, (4), I37-I52, I947.

RENAUD. - Apiculture et phytosociologie dans les Garrigues languedociennes. Ann. agron., I946.

RENNER (M.). - Ein Transozeanversuch zum Zeitsinn der Honigbiene. $\mathrm{Na}$ turwiss., 42, (r9), 540-I, I955.

RIBBANDS (C. R.). - The foraging method of individual honey bees. J. anim. Ecol., 18, 47-66, I949.

Ribbaxid (C. R.). - Division of labour in the honeybee community. Proc. roy. Soc. B, 140, 32-43, I952.

Ribbands (C. R.). - The flight range of the honeybee. J. anim. Ecol., 20, (2), 220-6, I95I-I952a.

RibBANDS (C. R.). - The relation between the foraging range of honeybees and their honey production. Bee World, 33, (1), 2-6, I952. $b$.

Ribiands (C. R.), Kalmus (H.) et Nixon (H. L.). - New evidence of communication in the honeybee colony. Nature, 1\%0,438, 1952. 
Ridi (M. S.) et Aboul Wara (M. H.). - An ostrogenic substance in pollen grains of date palm tree. Phoenix dactyfifera I. Palmae. Nature, 159, 409, 1947 .

Rösch (G. A.). - Arbeitsteilung im Bienenstaat. 2. /. vergl. Physiol., 2, (6), 57I-63I et 12, (I), I-7I, I925.

Rösch (G. A.). - Beobachtungen an Kittharz sammelnden Bienen (Apis mellifica I). Biol. Zentralblatt, 4\%, II3-I2I, I927.

Rösch (G. A.). - Bantätigkeit und Alter der Bienen. Arbeitsteilung. Z. $f$. vergl. Physiol., 6, (2), 264-298, I927 b.

Roubaud (E.). - La désocialisation chez l'abeille domestique (Apis mellifica L.). Coll. internat. C. N. R. S. Paris, I952, 34, 293-206, 1950.

SAgromsky (H.). - Uber einige Bestimmungen des Vitamins BI Gehaltes von Pollen mit Hilfe des Phycomyces-Testes. Biol. Zentr., 66, I40, I947.

SAKAGami (S. F.). - Untersuchungen über die Arbeitsteilung in einem $Z$ wergvolk der Honigbiene. Beiträge zur Biologie des Bienenvolkes, Apis mellifera I. I. Japanese Joumal of Zoology, 11, (I), II7-I85, I953a.

SAKAGAMI (S. F.). - Arbeitsteilung der Arbeiterinnen in einem Zwergvolk, bestehend aus gleichaltrigen Volksgenossen Beiträge zur Biologie des Bienenvolkes, Apis mellifera L. II. J. of the Fac. of Sc. Hokkaido University, Série VI, Zoology, 11, (3), 343-400, I053b.

SARKAR, WitTWER, LUECKE et SEI.I. - Quantitative estimation of some amino-acids in swet-corn pollen. Arch. Biochem., 22, (3), 353-350, I949.

Scrud (L.). - Untersuchungen iiber den Einfluss meteorologischer Elemente auf das Verhalten der Honigbiene (Apis mellifica L). Z. f. vergl. Physiol., 34, 258-277, 1952.

Schwan (B.) et Martinovs (A.). - Studier över binas (Apis mellifica) pollendrag i Ultuna. Statens Husdjursförsök Meddelande, no 57 , I954.

SchwaRz (I.) et Koch (A.). - Vergleichende Analyse der wichtigsten Wachstumsvitamine des Bliitenpollens, nebst einer Bemerkung iiber die Verteilung der Vitamine in Buchensämlingen. Wiss. Z. Univ. Halle, Math. Nat., 4, (I), 7-20, I954.

SingH (S.). - Behavior Studies of Honeybees in gathering nectar and pollen. Cornell Univ. Agric. Exp. Sta. Ithaca (N. Y.). Memoir 288, I950.

SLADEX (F. W. I.). - How pollen is collected by the social bees, and the part played in the process by the auricle. British Bee J., 39, 49I-493, IgII.

Siaden (F. W. L.). - Further Notes on how the corbicula is loaded with pollen. British Bee J., 40, I 44, I9I2.

SLAdiN (F. W. L.). - How pollen is collected by the honeybee. Nature, 88, 586-7, Igr $2 a$.

Sosa-Bourdouri (C.). - Sur la biochimie comparée des pollens et des spores des plantes vasculaires. Bull. soc. Chim. biol., 31, 69-7I, (I949).

SosA-Bourdouil (C.). - - Remarques sut la répartition des acides nucléiques à désoxypentose dans quelques organes reproducteurs des végétaux. C. R. Acad. Sc., 234, 2558, I952.

Sprenger (C. K.). - Das entdeckte Geheimnis der Natur im Bau und in der Befruchtung der Blumen. Berlin, Vieweg, I793.

STAPEL (C.) et ERIKSEN (K. M.). - Pollenanalytiske undersogelser over honningbiernes trackplanter. II. Tidskrift Planteave, 44, I29, I939.

Svoboda. - Uber den Wert des Pollens als Nahrungsmittel für Bienen. Schreiz. Bienen-Z., 4, I940. 